\title{
Evaluation of X-ray Spectroscopic Techniques for Determining Temperature and Density in Plasmas
}

Theodore Scott Lane

tslane@mix.wvu.edu

Follow this and additional works at: https://researchrepository.wvu.edu/etd

Part of the Plasma and Beam Physics Commons

\section{Recommended Citation}

Lane, Theodore Scott, "Evaluation of X-ray Spectroscopic Techniques for Determining Temperature and Density in Plasmas" (2019). Graduate Theses, Dissertations, and Problem Reports. 4018.

https://researchrepository.wvu.edu/etd/4018

This Dissertation is protected by copyright and/or related rights. It has been brought to you by the The Research Repository @ WVU with permission from the rights-holder(s). You are free to use this Dissertation in any way that is permitted by the copyright and related rights legislation that applies to your use. For other uses you must obtain permission from the rights-holder(s) directly, unless additional rights are indicated by a Creative Commons license in the record and/ or on the work itself. This Dissertation has been accepted for inclusion in WVU Graduate Theses, Dissertations, and Problem Reports collection by an authorized administrator of The Research Repository @ WVU.

For more information, please contact researchrepository@mail.wvu.edu. 


\title{
Evaluation of X-ray Spectroscopic Techniques for Determining Temperature and Density in Plasmas
}

\author{
Theodore Scott Lane
}
Dissertation Submitted to the Eberly College of Arts and Sciences at West Virginia University in partial fulfillment of the requirements for the degree of Doctor of Philosophy
in
Physics

\author{
Mark Koepke, Ph.D., Chair \\ Gregory Rochau, Ph.D. \\ Tudor Stanescu, Ph.D. \\ Jason Gross, Ph.D.
}

Department of Physics and Astronomy

Morgantown, West Virginia

2019

Keywords: Plasma, Spectroscopy, Stark Broadening, High Energy Density Copyright 2019 Theodore S. Lane 


\title{
Evaluation of X-ray Spectroscopic Techniques for Determining Temperature and Density in Plasmas
}

\author{
Theodore Scott Lane
}

\begin{abstract}
Temperature and density measurements of plasmas are important for understanding various phenomena. For example, equations of state, most scaling arguments for Inertial Confinement Fusion and laboratory astrophysics all rely upon accurate knowledge of temperature and density. Spectroscopy is a non-invasive technique to measure these quantities. In this work we establish a new spectroscopic technique by using it to determine temperature. We also compare and contrast the capability of two codes, PrismSPECT and ATOMIC, to infer electron density from experimentally acquired spectra via Stark broadening.

We compare and contrast the capability of isoelectronic line ratios and interstage line ratios in an absorption spectra to determine electron temperature of a plasma in Local Thermodynamic Equilibrium. The isoelectronic line ratio method has been used previously for experimentally required emission spectra. Stark broadening is often used with a tracer element to diagnose a plasmas electron density. Our objective is testing quantitatively how well Stark broadening models can predict the plasma density from multiple elements within the same plasma, and whether this is done self-consistently.

We measure a transmission spectrum through a $0.4 \mu \mathrm{m} \mathrm{Mg-NaF} \mathrm{multi-layered}$ foil, tamped with a uniform layer of $\mathrm{CH}$. We use X-rays from the $\mathrm{Z}$ facility at Sandia for heating and backlighting. Measurements were acquired in the $7-15 \AA$ range. We found that temperatures inferred from isoelectronic line ratios agree with temperatures inferred from inter-stage line ratios within error. We also found that densities inferred from different elements do not agree when using PrismSPECT, but do agree when using ATOMIC.
\end{abstract}


For my parents, Jay and Laura 


\section{Acknowledgements}

I would like to thank my thesis advisor Mark Koepke for the opportunities he's given me in pursuing plasma physics.

Thanks to Greg Rochau and Jim Bailey for their support of this work and support of graduate students working at Sandia and on the Z machine. Thanks also to Taisuke Nagayama for always being willing to help me with the more theoretical aspects of this work. Thanks to the $\mathrm{Z}$ facility staff who supported this project and had to deal with my mercurial nature and thus my last minute changes to just about everything.

Special thanks to Guillaume Loisel, without whom I don't think I would have been able to accomplish any of this work. I don't think I can single out any one part of this that you didn't help me with.

Thanks to Don Winget and CAPP for helping me survive Albuquerque.

Thanks to Igor Golovkin for helping me with the Prism suite of codes, helping me understand how they all work and for providing me with some great quotes. Thanks to Chris Fontes for running ATOMIC for us, especially when it was so last minute.

Thanks to Alex, Adrian, Kenneth and Joe for being great brewery buddies and even better friends. Thanks to Pedram, Cuyler, Dave, Kaustubh and Nick for putting up with my antics. Thanks to John for giving me antics to put up with. Thanks to Tristan and Dustin for allowing me to vent when we should have been working out. Thanks to Tommy and Pawel for being good friends, but also great soundboards 
and even better scientists, helping me learn the ways of coding and rigor.

Thanks to Kim and Phil for acting as a second family, and also for feeding me when times got tough.

Thanks to my parents for always supporting me and helping me through rough times.

Thanks to Krista for being there for me and really putting things in perspective.

\section{Funding Acknowledgments}

DE-NA0003874: Spectroscopic methods for obtaining plasma parameters applied to soft x-ray absorption spectra from radiatively heated Zpinch plasmas (PI: Koepke). Source of Support: DOE-NNSA (NNSASC Joint Program in HEDLP)

Project Title: SCGRF - Systematic study of spectral line formation in a multi-element plasma heated by z-pinch radiation (SCGSR-PI: T. S. Lane). Source of Support: DOE-SC-WDTS DE-SC0012515: WVUs contribution within the ZAPP collaboration on the Z Facility at Sandia National Laboratories (PI: Koepke). Source of Support: DOE-SC-FES (NSF-DoE Partnership in Basic Plasma Science and Engineering) 


\section{Contents}

1 Introduction 1

1.1 Motivation . . . . . . . . . . . . . . . . . 1

1.2 Layout of Thesis . . . . . . . . . . . . . . . . . 2

1.3 Spectroscopy as a means to document Atomic Kinetics . . . . . . 3

1.3.1 Line broadening . . . . . . . . . . . . . . . . . . 4

1.3.2 Local Thermodynamic Equilibrium . . . . . . . . . 8

1.3.3 Collisional-Radiative models . . . . . . . . . . . . . . . . 11

1.3.3.1 Radiative Transmission and Emission . . . . . . . 11

1.3.3.2 Photoionization and Radiative Recombination . . 13

1.3.3.3 Impact Excitation and De-excitation . . . . . . 15

1.3.3.4 Impact Ionization and Three-Body Recombination 18

1.3.3.5 Autoionization and Dielectric Recombination . . 21

2 Experiments $\quad 25$

2.1 The Z Machine and Dynamic Hohlraum . . . . . . . . . . . . 25

2.2 TIXTL . . . . . . . . . . . . . . . . . . 29

2.3 Experiments on the RCC . . . . . . . . . . . . . . . 32

2.3.1 Foils . . . . . . . . . . . . . . . 33

2.3.2 Z Shots . . . . . . . . . . . . . . . 35

2.3.3 Absorption Criterion . . . . . . . . . . . . . 35

2.3.4 Instrument Broadening . . . . . . . . . . . . . . . . 38

2.4 Data Processing . . . . . . . . . . . . . . . . . . . . . 41 
3 Determining the Usefulness of Isoelectronic Line Ratios as a Temperature Diagnostic $\quad 53$

3.1 Previous Work . . . . . . . . . . . . . . . . 53

3.2 Theory .............................. 54

3.2.1 Inter-Stage Line Ratios . . . . . . . . . . . . . . 55

3.2 .2 Isoelectronic Line Ratios . . . . . . . . . . . . . . 56

3.3 Line fitting - Experiment . . . . . . . . . . . . . . . . 57

3.4 PrismSPECT . . . . . . . . . . . . . . . . . 60

3.4.1 Line fitting - PrismSPECT . . . . . . . . . . 63

3.5 Analysis ....................... 65

3.6 Results . . . . . . . . . . . . . . . . . . 67

3.6 .14 micron tamped . . . . . . . . . . . . . . 67

3.6.2 7 micron tamped . . . . . . . . . . . . . . 69

3.6 .315 micron tamped . . . . . . . . . . . . . . . 73

3.6.4 Overall results . . . . . . . . . . . . . . . . . . . . . 75

4 Stark Effect on Multiple, Multiply-Ionized Elements $\quad 77$

4.1 Theory . . . . . . . . . . . . . . . . . . 77

$4.1 .1 \quad$ PrismSPECT . . . . . . . . . . . . . 78

4.1 .2 ATOMIC . . . . . . . . . . . . . 80

4.2 Fitting the Data . . . . . . . . . . . . . . . . 82

4.3 PrismSPECT fitting . . . . . . . . . . . . . . . . . . . . 82

4.4 ATOMIC fitting . . . . . . . . . . . . . . . . 86

4.5 Analysis . . . . . . . . . . . . . . . . . 90

4.6 Results . . . . . . . . . . . . . . . . . . . . . 91

4.6 .14 micron Tamped . . . . . . . . . . . . . . . . . . . 93

4.6 .27 micron Tamped . . . . . . . . . . . . . . . . 95

4.6.3 Causes of Discrepancies . . . . . . . . . . . . . . . 99

5 Summary, Conclusions and Future Work 101

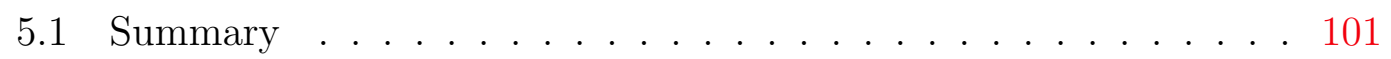

5.2 Conclusions . . . . . . . . . . . . . . . . . . . . 102

5.3 Future Work . . . . . . . . . . . . . . . . . . 105

5.3.1 Investigation of more Codes . . . . . . . . . . . 105 
5.3 .2 Density Gradients . . . . . . . . . . . . . . . . . . 105

5.3.3 Temperature Gradients . . . . . . . . . . . . . . . . . 108

5.3.4 Temporal Evolution . . . . . . . . . . . . . . 110

$\begin{array}{ll}\text { A Z Shot Dates } & 113\end{array}$

B List of Presentations 115

$\begin{array}{lr}\text { C Example of Code } & 119\end{array}$

$\begin{array}{lr}\text { References } & 123\end{array}$ 


\section{1}

\section{Introduction}

There is no lift to success, you have to climb the stairs.

-Emil Oesch

\subsection{Motivation}

This thesis will focus on two things; comparing and contrasting the ability of isoelectronic line ratio methods to determine plasma electron temperature for Local Thermodynamic Equilibrium (LTE) conditions, and comparing and contrasting the ability of the Collisional-Radiative spectroscopy codes PrismSPECT and ATOMIC determine electron density via Stark broadening of spectral lines across multiple elements within the same plasma. Isoelectronic line ratios have been previously used in emission spectroscopy to determine plasma temperature (1). Our objective is to establish this technique for absorption spectra. This would allow absorption experiments to benefit from isoelectronic line ratios the same way emission experiments do, such as tailoring the stoichiometry of a sample to increase signal-to-noise ratio and to allow for temperature measurements within specific wavelength ranges. We conclude that we are the first to establish this technique in absorption

Stark broadening is often one of the only diagnostics to determine electron density in hot dense plasmas (2), (3), (4), (5), (6). Due to the complexity of Stark broadening model equations and solutions, codes are often employed to synthesize 


\section{INTRODUCTION}

spectra to compare to experimentally acquired spectra and yield electron density. However, it has been found (7) that there is discrepancies larger than twenty-five percent between different Stark broadening models. As a first order investigation, we aim to create a plasma with four different elements and see if the inferred electron density is the same for three of these elements. We will compare and contrast the collisional-radiative models PrismSPECT and ATOMIC in this way.

\section{$1.2 \quad$ Layout of Thesis}

This thesis is the combination of research done individually and collaboratively. Since no results from this dissertation have been published yet, explicit statements will be incorporated for attributing credit to effort and accomplishments. This chapter explains the content described in later chapters.

The remainder of this chapter will review pertinent information regarding spectroscopy and collisional-radiative code calculations.

Chapter 2 describes the experimental set up used in our investigations. The $\mathrm{Z}$ machine, used in these experiments, is discussed, along with how this experiment in particular was fielded. The diagnostic techniques used to capture spectra are outlined. Data processing and analysis are documented thoroughly.

Chapter 3 establishes the methodology of the isoelectronic line ratio technique. This section provides the theory behind this technique and behind the inter-stage ratio technique used for comparison. The results of these techniques are presented at the end of the chapter.

Chapter 4 is about multi-element Stark broadening. The Stark broadening models used by PrismSPECT and ATOMIC are discussed, and the data is interpreted.

Chapter 5 summarizes the overall results, including possible shortcomings and possible future work. 

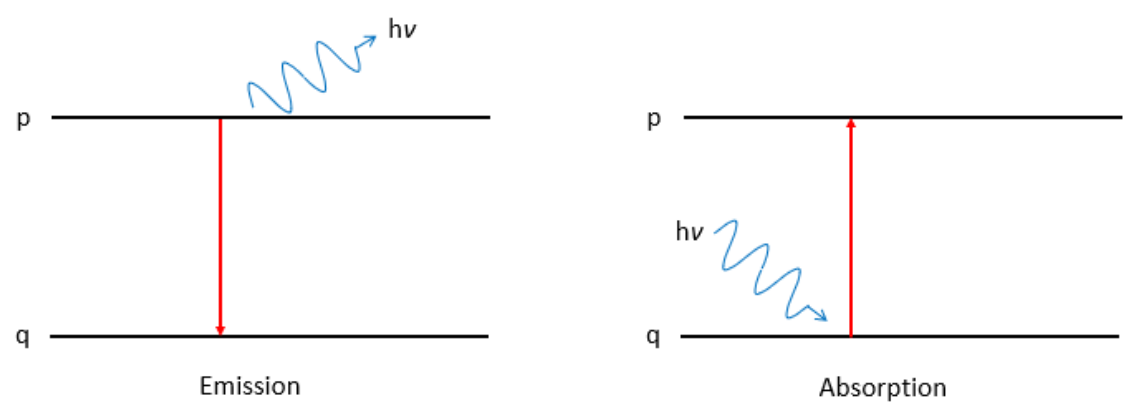

Figure 1.1: In photon emission, an electron in excited state $\mathrm{p}$ decays down to lower energy state q releasing a photon with energy $h \nu$. Absorption is this process in reverse, an electron in lower state $\mathrm{q}$ absorbs a photon and enters the excited state $\mathrm{p}$.

\subsection{Spectroscopy as a means to document Atomic Kinetics}

In general atomic kinetics is defined as the interactions between radiation and matter (8). The scope of this project is limited to absorption and emission as phenomena responsible for x-ray spectra. Absorption and emission spectroscopy are processes by which a photon is emitted or absorbed by atoms due to an electron transitioning from an upper or lower level to a lower or upper level, respectively (figure 1.1). For emission, the transition from an upper state back down to a lower state emits a photon of specific energy $\delta E$ equal to the energy difference of the upper and lower states. Absorption is the reverse process.

Whereas emission is a passive measurement, only requiring a finite temperature atom and optically thin conditions. Absorption is an active measurement, requiring a backlighter, a broadband radiation source in which the bound electrons are bathed so that they can excite to upper levels.

One can consider a radiation source with intensity $I_{\lambda}(x)$ that is incident upon a collection of particles with spatial extent $d x$, where the $\lambda$ subscript denotes that $I(x)$ is a function of photon wavelength. The amount of radiation that passes 


\section{INTRODUCTION}

through the ensemble of atoms over distance $d x$ can be expressed as

$$
I_{\lambda}(x+d x)=I_{\lambda}(x)-\kappa_{\lambda} I_{\lambda}(x) d x+\eta_{\lambda} d x .
$$

Here the absorption coefficient $\kappa_{\lambda}$ describesthe incremental intensity of light absorbed by the atoms across the distance $d x$ at a specific wavelength. Similarly, the emission coefficient $\eta_{\lambda}$ is the amount of light that is emitted by the atoms over the distance $d x$ at a specific wavelength (9). Integrating this equation over a uniform medium of particles leads to

$$
I_{\lambda}(x)=I_{\lambda}(x) e^{-\kappa_{\lambda} x}+\frac{\eta_{\lambda}}{\kappa_{\lambda}}\left(1-e^{-\kappa_{\lambda} x}\right) .
$$

Here, the exponent $\kappa_{\lambda} x$ is commonly defined as the optical depth $\tau_{\lambda}$ and the

fraction $\frac{\eta_{\lambda}}{\kappa_{\lambda}}$ is referred to as the Source function $S_{\lambda}$ which is equal to the Blackbody radiation emitted by the ensemble of atoms. Both $\kappa_{\lambda}$ and $\eta_{\lambda}$ are wavelength dependent.

For this dissertation, interactions within a system composed of ions and electrons can be from free electrons accelerating, called free-free emission, electrons being captured, called bound-free interactions, and an electron transitioning between states like in an atom, called bound-bound interactions. Before and after a bound-bound interaction, there can be vacancies in electron shells. This means that not only are the energy levels shifted from the case of a neutral atom, but there are also more possible transitions, as the electron can transition to a bound state that would normally be occupied in a neutral atom.

\subsubsection{Line broadening}

Otherwise zero-width transition lines undergo broadening, where they are convolved with Gaussian, Lorentzian or Voigt profiles to provide width to the transition line. There are three main causes of line broadening in plasmas; natural line broadening (10), Doppler line broadening (9) and stark broadening (11). Natural line broadening is the intrinsic width a spectral line has due to the Heisenberg uncertainty principle.

$$
\frac{\hbar}{2}=\Delta E \Delta t
$$




\subsection{Spectroscopy as a means to document Atomic Kinetics}

where $\hbar$ is Plank's constant divided by $2 \pi, \Delta t$ is the uncertainty in state lifetime and $\Delta E$ is the uncertainty in state energy. For the case of a transition that results in a spectral line, $\Delta t$ is the lifetime of the transition. This is the inverse of the Einstein coefficient for the transition

$$
A_{i j}=\frac{1}{\Delta t}
$$

This tells us that the line broadening in energy is (10)

$$
\Delta E=\frac{\hbar}{2} A_{i j}
$$

As $\Delta E$ is line dependent, the value for this broadening will vary from line to line. Due to this being a quantum mechanical feature, it is the smallest contribution to line width. In general natural broadening is seen to have a Lorentzian in shape.

Doppler broadening is an ensemble effect caused by motion of emitting ions within a plasma. Motion from an emitting ion causes a Doppler shift, which is given by (12)

$$
\frac{\omega-\omega_{i j}}{\omega_{i j}}=\frac{v_{x}}{c}
$$

where $\omega_{i j}$ is the angular frequency of the emitted photon, $\omega$ is the frequency it appears at, $c$ is the speed of light and $v_{x}$ is the speed of the emitter. In the case of a Maxwellian plasma distribution for velocities, the line becomes broadened according to

$$
I(\omega)=\frac{1}{\Delta \omega_{D} \sqrt{\pi}} e^{-\left(\frac{\omega-\omega_{0}}{\Delta \omega_{D}}\right)^{2}},
$$

where $\Delta \omega_{D}$ is the Doppler broadened width, given by

$$
\Delta \omega_{D}=\omega_{0}\left(\frac{2 k_{B} T_{i}}{M_{i} c^{2}}\right)^{1 / 2}
$$

where $T_{i}$ is the temperature of the ion population and $M_{i}$ is the mass of the ion (9). It should be noted that equation 1.7 is a Gaussian distribution, meaning 


\section{INTRODUCTION}

that the Doppler width is a Gaussian width, with the width for a particular line being given by

$$
\Delta \lambda_{D}=\lambda_{0} \frac{2 T_{i} \ln (2)}{M_{i} c^{2}} .
$$

Here, $T_{i}$ should be in $\mathrm{eV}$, as should $M_{i} c^{2}$, which is the rest mass of the ion. We've also converted to wavelength for future purposes (13).

Stark broadening is a consequence of the Stark effect. The Stark effect is a spectral shift in energy (or wavelength) due to an electric field. Plasma supports abundant electric fields due to being quasi-neutral (11). The broadening of a line can be exactly calculated by the shift in the Hamiltonian of the emitting ion (12), given by

$$
\begin{gathered}
\Delta H=-\operatorname{er} E(t), \\
E(t)=\frac{1}{4 \pi \epsilon_{o}} \sum_{i} z_{i} e \frac{r_{i}(t)}{r_{i}(t)^{3}}-\frac{1}{4 \pi \epsilon_{o}} \sum_{j} e \frac{r_{j}(t)}{r_{j}(t)^{3}},
\end{gathered}
$$

where $\mathrm{i}$ and $\mathrm{j}$ refer to ions and electrons respectively. This formalism is formidable to solve, as it would require knowledge of exact locations of all particles in time. Impact approximation and quasi-static approximation are two main approximations that can be made to determine Stark broadening contributions. The Impact approximation dominates isolated lines in atoms and ions, with its contributions being even larger for ions (11). As we'll only be investigating isolated lines from the He-like sequence in this thesis, where the impact approximation dominates, we shall not consider the quasi-static approximation.

In the impact approximation, line shifts are caused by electron collisions with the emitting ion (figure 1.2). When there are multiple collisions, or multiple emitting ions undergoing different collisions, then this leads to the broadening of a line (figure 1.3).

Within the impact approximation, there are two different types of collisions, strong and weak collisions. Collisions with large impact parameters are considered weak, and while the effects of one collision is small, there are more collisions with large impact parameters, and thusly they dominate Stark broadening. When 


\subsection{Spectroscopy as a means to document Atomic Kinetics}
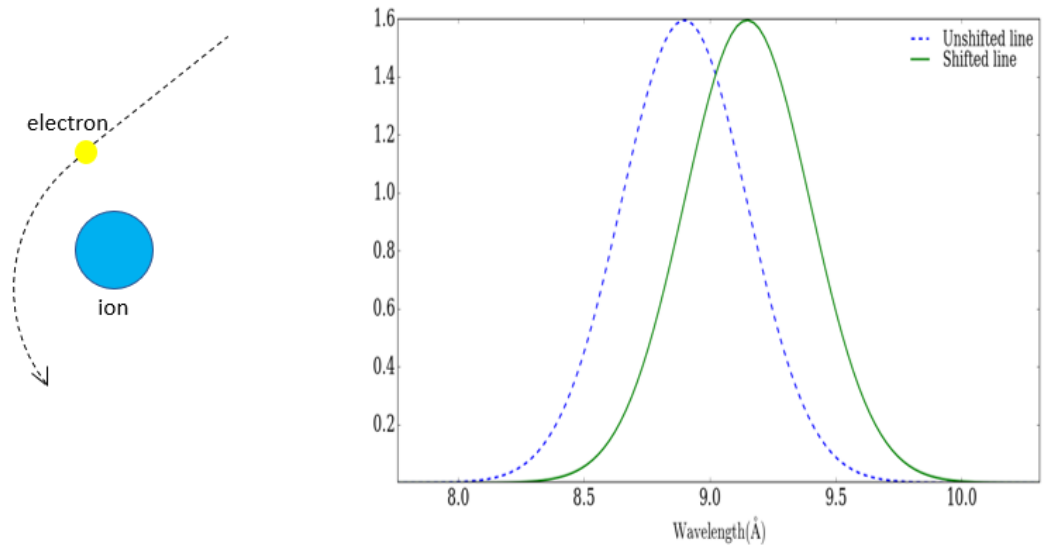

Figure 1.2: An electron colliding with an emitting ion will have an electric field that interacts with the emitter. This causes a shift in the central wavelength of the emitted spectral line.
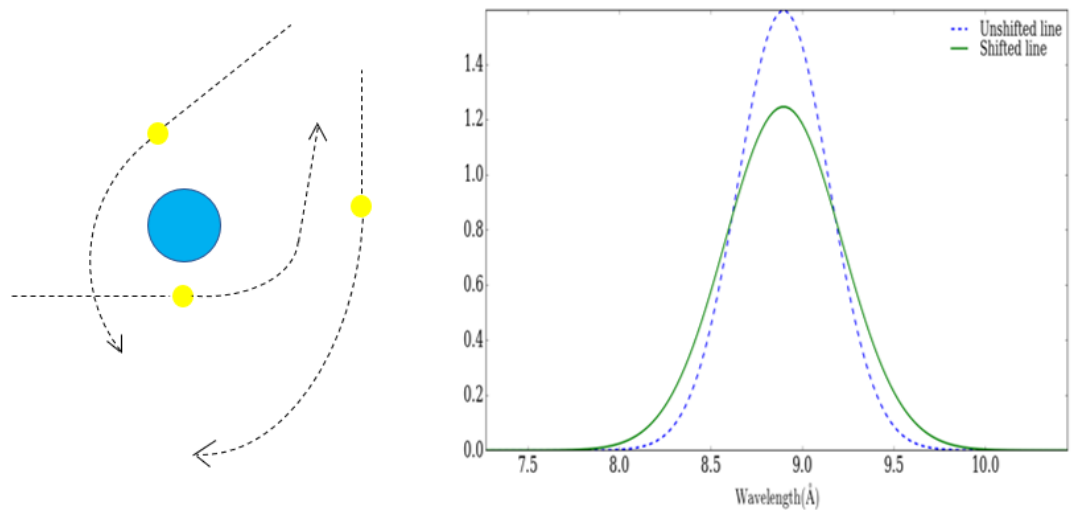

Figure 1.3: In a dense plasma, multiple collisions can occur, all of which shift the central wavelength. When taking into account multiple emitters with multiple collisions, the overall effect is to broaden the spectral line. 


\section{INTRODUCTION}

impact parameters are the size of the Weisskopf radius or smaller the effects of the impact are considered strong, but these collisions are rare. Baranger (14) has a starting point for calculating the FWHM of Stark broadening in angular frequency, given by

$$
\begin{array}{r}
\Delta \omega_{S}=n_{e} \int_{0}^{\infty} v f_{e}(v)\left(\sum_{i^{\prime} \neq i} \sigma_{i^{\prime} i}(v)+\sum_{j^{\prime} \neq j} \sigma_{j^{\prime} j}(v)\right) d v+ \\
n_{e} \int_{0}^{\infty} v f_{e}(v)\left(\int\left|\phi_{i}(\theta, v)-\phi_{j}(\theta, v)\right|^{2} d \Omega\right) d v
\end{array}
$$

where the first term is the contributions from inelastic collisions, with crosssections $\sigma, \mathrm{i}$ and $\mathrm{j}$ refer to the upper and lower states and this is integrated over the velocity distribution function of the plasma. The second term is contributions from elastic scattering, with $\phi$ being the elastic scattering amplitude for the respective states and integration is over the scattering angle $\theta$ through the solid angle component $d \Omega$. This is just the starting point for many Stark broadening models, as models often use different assumptions concerning the scattering amplitudes and how to approach the scattering cross-sections. The models behind PrismSPECT and ATOMIC's Stark broadening will be discussed more later.

\subsubsection{Local Thermodynamic Equilibrium}

Within a plasma, or any distribution of particles, there are four primary thermodynamic regimes; Thermodynamic equilibrium, local thermodynamic equilibrium, partial local thermodynamic equilibrium and non-local thermodynamic equilibrium (9). The easiest to work with theoretically is that of thermodynamic equilibrium; where the particles and the radiation all have the same temperature, population densities follow the Boltzmann distribution and a transition process and it's inverse are equal (known as detailed balance).

Particles (both electrons and ions) follow a Maxwell-Boltzmann distribution:

$$
f(v)=\left(\frac{m}{2 \pi T}\right) e^{-\frac{m v^{2}}{2 T}}
$$




\subsection{Spectroscopy as a means to document Atomic Kinetics}

where $v$ is the magnitude of a particle's velocity vector, $m$ is the mass of the particle and $T$ is the characteristic temperature of the system in energy units. The Maxwell-Boltzmann distribution can also be written in energy units as

$$
f(E) d E=2\left(\frac{E}{\pi}\right)^{1 / 2}(T)^{-3 / 2} e^{-\frac{E}{T}} d E,
$$

where $E$ is the energy of a particle. The integrals of these functions by definition must equal one when integrated over the entire possibilities of velocity and energy. The electron energy distribution function (EEDF) is important for calculating excitation and de-excitation rates.

The power radiated from a source with temperature $\mathrm{T}$ as a function of frequency $\nu$ is defined as the Planckian distribution (15)

$$
E_{\nu}(\nu, T)=\frac{2 h}{c} \frac{\nu^{3}}{e^{\frac{h \nu}{T}}-1},
$$

with $\nu$ being the frequency of light, $h$ is Planck's constant and $c$ is the speed of light.

The excited states of particles in thermodynamic equilibrium follow the Boltzmann distribution (12) for levels within a charge state

$$
\frac{n_{z}(p)}{n_{z}(q)}=\frac{g_{z}(p)}{g_{z}(q)} e^{-\frac{E_{z}(p)-E_{z}(q)}{T}}
$$

where $n_{z}(s)$ is the density of ions in charge state $\mathrm{z}$ in state $\mathrm{s}, g_{z}(s)$ is the degeneracy of state $\mathrm{s}$ and $E_{z}(s)$ is the energy of an ion with charge state $\mathrm{z}$ in state $\mathrm{s}$. In thermodynamic equilibrium, the charge state distribution function follows the Saha-Boltzmann equation

$$
\frac{n_{z+1}(g) n_{e}}{n_{z}(q)}=2 \frac{g_{z+1}(g)}{g_{z}(q)}\left(\frac{m_{e} T}{2 \pi \hbar^{2}}\right)^{3 / 2} e^{-\frac{E_{z}(c)-E_{z}(q)}{T}},
$$

with $E_{z}(c)-E_{z}(q)$ equal to the energy it would take to transition an electron in state $\mathrm{q}$ to a continuum state, ionizing the ion further (13).

Total thermodynamic equilibrium is rare experiment, because radiation can escape a plasma quickly and easily, resulting in the radiation temperature being higher than that of the ions and electrons that make up the plasma. At 


\section{INTRODUCTION}

high enough particle densities, collisions will dominate the interactions within a plasma, resulting in temperature equilibrium between the electrons and ions. This is called Local Thermodynamic Equilibrium (LTE). LTE exists if the radiation properties and the plasma is collision dominated. LTE is a condition under which matter emits radiation according to its intrinsic properties and its temperature, uninfluenced by the magnitude of any incident radiation. An example is that for a plasma composed of $\mathrm{Al}$ ions at temperatures between 10 and $1000 \mathrm{eV}$, the mean free path of photons is 300 times larger than for the electrons and ions (13). Within a plasma, since electrons move much faster than ions at the same temperature, electron collisions dominate in LTE and will be what establishes temperature equilibrium, even though ion-ion collisions transfer more momentum per event.

A simple way criterion for LTE, proposed by Griem (16) is when the electron collision rate across the largest energy gap in the plasma system is larger than the transition rate for the largest energy gap by a factor of ten. This would mean that the population distribution would differ from a Boltzmann distribution by less than ten percent. This criteria can be expressed as a simple formula (17)

$$
n_{e}=1.4 \times 10^{20}\left(E_{z}(p)-E_{z}(q)\right)^{3} T_{e}^{1 / 2},
$$

where if $E_{z}$ and $T_{e}$ are expressed in $\mathrm{eV}$, then $n_{e}$ is expressed in $m^{-3}$. In the plasmas discussed in this thesis, the highest energy transition is that of Hydrogenlike Oxygen, specifically the Lyman $-\alpha$. This transition has a transition energy of $E_{z}(p)-E_{z}(q) \simeq 18.97$ (18). If we assume a temperature of $100 \mathrm{eV}$ (a factor of two larger than is likely from experiments) and solve for the electron density, we find that $n_{e}>9.5 \times 10^{18} \mathrm{~cm}^{-3}$ must exist in order to claim LTE. This is smaller than all of the densities we will be testing for or assume our plasmas to have by two orders of magnitude, and was achieved with a higher temperature than is likely. This justifies the LTE approximation for the Collisional-Radiative models employed in these experiments. 


\subsection{Spectroscopy as a means to document Atomic Kinetics}

\subsubsection{Collisional-Radiative models}

Within a plasma, transitions among quantum states can be caused by a multitude of reasons, the two most important being collisional processes and radiative processes. For this reason, when calculating synthetic spectra of a plasma, Collisional-Radiative (CR) codes are often employed. These codes calculate the rate coefficients for the different atomic processes; radiative transmission and emission, electron impact excitation and de-excitation, radiative recombination and photoionization, electron impact ionization and three-body recombination. The aforementioned rate coefficients are the likely-hood of one of these processes occurring at a given moment in the plasma. Once the rate coefficients are calculated, the code can then populate the states accordingly and produce emission or absorption spectra.

This section is meant as an overview of how to calculate rate coefficients, and is by no means definitive. Many of these are still an active area of research. We are pulling from the texts of Salzman, Fujimoto and Kunze (13), (9) and (12) and supplementing with papers where it is applicable.

\subsubsection{Radiative Transmission and Emission}

As discussed earlier, when a bound electron undergoes a transition from state p (upper) back to state $q$ (lower) it emits a photon with characteristic energy $h \nu_{q p}$. How often this decay occurs is dependent on how many ions are in the state $\mathrm{p}$, and this occurs spontaneously, meaning there must be some amount of time dependence to this. The equation governing this process is given as

$$
-\frac{d n_{z}(p)}{d t}=A_{p q} n_{z}(p)
$$

such that the amount of particles leaving state $\mathrm{p}$ as a function of time must be equal to the rate at which particles can leave state $\mathrm{p}$ times the number of particles in state p. Here, $A_{p q}$ is that rate, and in this case it is the Einstein coefficient for emission, given by

$$
A_{p q}=\frac{2 \pi e^{2} \nu^{2} g(q)}{m c^{3} \epsilon_{o} g(p)} f_{q p}
$$


where $g(q)$ and $g(p)$ are the statistical weights of the lower and upper states, and $f_{q p}$ is the absorption oscillator strength. The absorption oscillator strength is a quantum mechanical quantity that depends on the electric dipole moment of the of the upper and lower states. This is a quantity that is hard to calculate, fortunately the National Institute of Standards and Technology (NIST) has a database of calculated and measured oscillator strengths available on the internet (19).

When an external electromagnetic field is present, the field can induce both emission and absorption. The rate equations for these is given by

$$
\begin{gathered}
-\frac{d n_{z}(p)}{d t}=B_{p q} u_{\nu} n_{z}(p), \\
-\frac{d n_{z}(q)}{d t}=B_{q p} u_{\nu} n_{z}(q),
\end{gathered}
$$

where $u_{\nu}$ is the external electromagnetic field and $B_{p q}$ and $B_{q p}$ are the Einstein coefficients for induced emission and absorption respectively (12). An important point is that the external electromagnetic field should be regarded as constant over the energies of the transition. The Einstein B coefficients can be related to the Einstein A coefficients by

$$
\begin{gathered}
g(p) B_{p q}=g(q) B_{q p}, \\
A_{p q}=\frac{8 \pi h \nu^{3}}{c^{3}} B_{p q} .
\end{gathered}
$$

The probability of absorption can also be related to the opacity of the plasma, as opacity is the likely-hood of a photon being absorbed after traveling $\Delta x$ through a plasma. This can be seen by

$$
B_{q p} u_{\nu} n_{z}(q) \Delta x h \nu=\kappa_{L} \Delta x \frac{u_{\nu}}{c},
$$

when simplified this yields

$$
B_{q p} n_{z}(q) \frac{h \nu}{c}=\kappa_{L}
$$




\subsection{Spectroscopy as a means to document Atomic Kinetics}
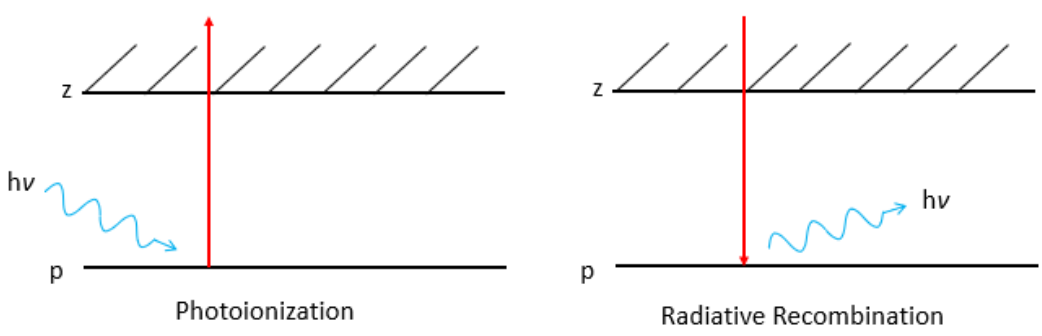

Figure 1.4: Photoionization occurs when an electron in $\mathrm{p}$ absorbs a photon with energy $h \nu$ which is high enough to send the electron into one of the continuum states, effectively having it leave the ion, making the ion go from charge state Z-1 to $\mathrm{Z}$. The reverse of this process is called radiative recombination.

where $\kappa_{L}$ is the line integrated opacity. This allows for the relation of number of particles in a lower state $\mathrm{q}\left(n_{z}(q)\right)$ to be directed related to the integral of a spectral line $\left(\kappa_{L}\right)$.

\subsubsection{Photoionization and Radiative Recombination}

Within plasmas, there is the chance that a photon will further ionize an ion (photoionization) or that an ion will capture an electron and release a photon of appropriate energy (Radiative recombination), this can be seen in figure 1.4.

Since these transitions depend on the electron reaching a continuum state with some energy $E_{\text {kinetic }}$ they can often be hard to calculate. Going off of detailed balance, we can equate the two rates in order for balance to be maintained. As photoionization is easier to calculate we shall start there. First, we can define the cross-section for absorption as (12)

$$
\sigma_{L}(\nu)=\pi c r_{e} f_{q p}
$$

This is simply the absorption coefficient for a state divided by the number of ions in that state. This is for transitions between bound states however, and the upper state in photoionization is continuous, not discrete. Due to this, the oscillator strength must be taken as a continuous function and differentiated as a function 


\section{INTRODUCTION}

of energy, $\frac{d f_{q p}}{d E_{\text {kinetic }}}$. However, the differential of energy in the case of a photon is

$$
d E_{\text {kinetic }}=h d \nu
$$

This means that the continuous derivative of the oscillator strength goes as

$$
\frac{d f_{q p}}{d E_{\text {kinetic }}}=\frac{d f_{q p}}{h d \nu}
$$

which can then be substituted in to our expression for the cross-section, giving a cross-section for photoionization (20)

$$
\sigma_{L}(\nu)=\pi c r_{e} \frac{d f_{q p}}{d \nu}
$$

This system is hard to calculate generically, involving different quantum mechanical waveforms for every different ion. For Hydrogen-like ions, the solution can be simplified to $(21)$

$$
\sigma_{K r}=\frac{64 \alpha Z^{4} E_{R}^{3} \pi a_{o}^{2}}{3 \sqrt{3} n_{q}^{5}(h \nu)^{3}},
$$

where $\alpha$ is the fine structure constant, $E_{R}$ is the Rydberg energy, $a_{o}$ is the Bohr radius and $n_{q}$ is the population of state q. $\sigma_{K r}$ is often referred to as the Kramer cross-section, after the physicist who discovered it. For non-Hydrogen-like ions, the results of Pratt et al (22) are often used. As that work is a book all on its own we won't go into further detail here.

In order to go from photoionization rates to radiative recombination rates, one just needs to use the Milne relation (23)

$$
\frac{\sigma_{\text {photo }}(z, q)}{\sigma_{r r}(z+1)}=\frac{2 m c^{2} E_{k i n e t i c} g_{z+1}}{(h \nu)^{2} g_{z, q}} .
$$

Here, $\sigma_{\text {photo }}(z, q)$ is the cross-section for the photoionization of an ion in state $\mathrm{q}$ and charge state $\mathrm{z}$, while $\sigma_{r r}(z+1)$ is the cross-section for radiative recombination of an ion with charge state $\mathrm{z}+1$ into the charge state $\mathrm{z}$ and state $\mathrm{q}$. Thus if the photoionization cross-section can be calculated, the radiative recombination can be calculated. 


\subsection{Spectroscopy as a means to document Atomic Kinetics}

Once the cross-section is known, the rates for these processes can be calculated. For a beam of electrons all with the same speed, $v$, the radiative recombination rate would be

$$
\sigma_{r r} v
$$

However, in standard plasmas a beam of electrons is not the case. In those situations, one must integrate over the energy distribution function to get the radiative recombination rate, $\beta$

$$
\beta=\int_{0}^{\infty} \sigma_{r r}(z+1) v f(E) d E
$$

where $f(E)$ is the energy distribution function for the electrons. The radiative recombination rate must be calculated for each different $\sigma_{r r}$, but computers can accomplish this relatively quickly. Multiplying the rate by the amount of ions in a charge state $\left(n_{z+1}\right)$, the amount of electrons that can combine $\left(n_{e}\right)$ and the relative weights of the charge state $(g(q))$, then the time evolution of photoionization and radiative recombination can be calculated.

\subsubsection{Impact Excitation and De-excitation}

Important processes that occur that can populate or depopulate an upper state in an ion without a photon interaction are impact excitation and de-excitation. Impact excitation is when an electron with some energy $E_{\text {kinetic }}$ collides with an ion, causing a bound electron to jump into an excited state and the electron to now have an energy of $E_{\text {kinetic }}-\Delta E$, where $\Delta E$ is the energy of the bound-bound transition (figure 1.5). The inverse of this process is impact de-excitation, with the free electron leaving with more energy than when it started. It is important to note that we are only discussing transitions to another state within the same charge state, ionization by impact will be discussed later. The excitation and de-excitation rates can be connected through

$$
\frac{D}{X}=\frac{g(q)}{g(p)} e^{\frac{-\Delta E}{T_{e}}},
$$




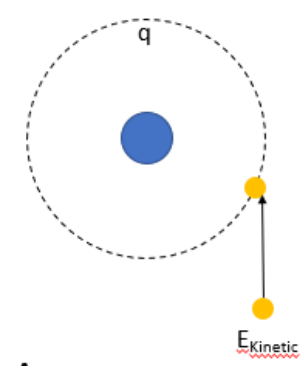

A

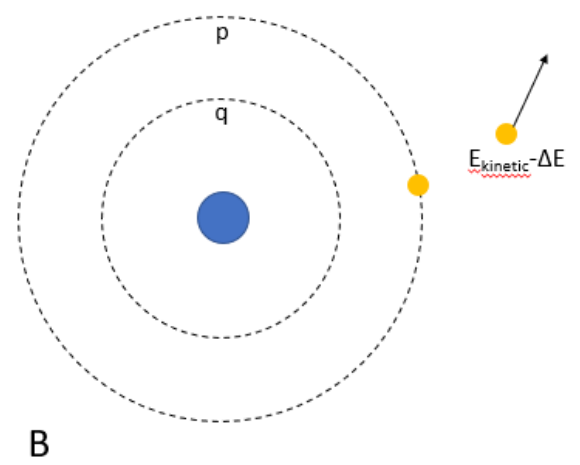

Figure 1.5: Impact Excitation: (A) An electron with some energy $E_{\text {kinetic }}$ collides with an ion in state q. (B) This transfers some of the energy to the ion, allowing it to enter upper state $\mathrm{p}$, while the free electron now has a loss of energy, where $\Delta E$ is equal to the energy required for the transition.

where $D$ and $X$ are the de-excitation and excitation rates. In this manner, only one rate need be calculated. The relation between cross section and the rates will be employed in our calculations,

$$
\begin{gathered}
D=<\sigma_{D} v>, \\
X=<\sigma_{X} v>.
\end{gathered}
$$

This relationship is due to the inclusion of free electrons in the rates, forcing calculations to rely on the electron distribution function, which in turn results in different assumptions being made.

It is important to mention that in many papers and books discussing impact excitation and de-excitation, the parameter that is often quoted is the collision strength, $\Omega_{z ; p, q}$. The reason for this is because the collision strength is independent of whether it the process is excitation or de-excitation such that $\Omega_{z ; p, q}=\Omega_{z ; q, p}$. The collision strength is related to the cross-section by (13)

$$
\sigma_{X}(z, q)=\pi a_{o}^{2} \frac{E_{R}}{g(q) E_{\text {kinetic }}} \Omega_{z ; q, p}
$$

where $a_{o}$ is the Bohr radius and $E_{R}$ is the Rydberg energy for Hydrogen. 


\subsection{Spectroscopy as a means to document Atomic Kinetics}

Much like in the preceding section, there are several different models to approximate impact excitation. Many of these models have been tested against experimental results collected from Electron Beam Ion Trap (EBIT) devices, that contain an ion in a magnetic trap and then fire an electron beam at them. For the purpose of this dissertation I will discuss the more widely used models; the Van Regemorter model for Hydrogen-like ions and the Sampson model for ions that are Helium-like to Boron-like (2-5 remaining bound electrons) for all Z.

Van Regemorter's rate coefficient (24) is one of the older models for impact excitation. It has been tested against newer models and been found to be accurate. The model is based on a comparison between experimental results and the first order Born approximation, and is given by

$$
X(q, p)=3.2 \times 10^{-7}\left(\frac{E_{R}}{T_{e}}\right)^{3 / 2} f_{q p} \frac{e^{\frac{-\Delta E}{T_{e}}}}{\frac{\Delta E}{T_{e}}} G .
$$

Here $f_{q p}$ is the oscillator strength for a transition from $\mathrm{q}$ to $\mathrm{p}$, and most importantly $G$ is a Gaunt factor, in this case found to best be $G=0.8$ (25) when

$\frac{\Delta E}{T_{e}}>1$ and $G=\frac{\sqrt{3} E_{1}}{2 \pi}$ when $\frac{\Delta E}{T_{e}}<1$; here $E_{1}=\int_{\frac{\Delta E}{T_{e}}}^{\infty} d t e^{-t} / t$. A Gaunt factor is a correction factor meant to transition classical assumptions into a quantum mechanical setting.

This model is not very useful for the experiments conducted in this thesis; there are no Hydrogen-like ions seen in the data. The primary transitions seen from spectra are from Helium-like through Beryllium-like charge states. Many codes employ the Sampson model (25) in order to calculate impact excitation rates. This model uses a Coulomb-Born approximation, which considers the electron as a plane wave, with the positive ion giving a long-range Coulomb interaction which is treated like a time-dependent perturbation. The parametrization of the collision strength is given by

$$
\Omega_{z ; q, p}=c_{o}+\frac{c_{r}}{\left(a+\frac{E_{k i n e t i c}}{\Delta E}\right)^{r}}+\frac{c_{r+1}}{\left(a+\frac{E_{\text {kinetic }}}{\Delta E}\right)^{r+1}}+\frac{4}{3} Z^{2} \ln \left(\frac{E_{\text {kinetic }}}{\Delta E}\right) S .
$$

Here, $S$ is the spin quantum number of the ion, $r=1$ for classically allowed transitions and $r=2$ for cases where $Z^{2} S=0$ and $c_{0}, c_{1}, c_{2}$ and $a$ are all fitting 


\section{INTRODUCTION}

parameters. These fitting parameters depend on which transition is being considered, and so are too lengthy to be listed here (26), (27) and (28) . By assuming a Maxwellian velocity distribution and averaging the cross-section and the velocity, the rate of impact excitation for Sampson's model follows

$$
\begin{array}{r}
X_{Z}(q, p)=\frac{E_{R} \pi a_{o}^{2}}{T_{e} g_{q} Z_{\text {eff }}^{2}}\left(\frac{8 T_{e}}{\pi m}\right)^{(1 / 2)} \\
{\left[c_{0} e^{\frac{\Delta E}{T_{e}}}+\frac{4}{3} Z^{2} S E_{1}+\frac{\Delta E}{T_{e}} e^{a \frac{\Delta E}{T_{e}}}+\left(\frac{c_{r}}{(a+1)^{r-1}} E_{r}+\frac{c_{r+1}}{(a+1)^{r}} E_{r+1}\right)\right]}
\end{array}
$$

Here $Z_{e f f}=Z-z$ is the effective charge after screening electrons are taken into account, with $z$ equal to the remaining electrons in the charge state, $E_{1}$ is the same as before, and $E_{r}=\int_{(a+1) \frac{\Delta E}{T_{e}}}^{\infty} d t e^{-t} / t^{r}$.

An important note is that the above models are solely for single-electron impact excitation and de-excitation. Multi-electron impact excitation is much more complicated than what has been discussed up to this point, and the models are more hotly contested. For the experiments in this thesis, only single-electron impact broadening need to be considered, as the electron densities discussed are less than $10^{23} \mathrm{~cm}^{-3}(13)$.

\subsubsection{Impact Ionization and Three-Body Recombination}

A process closely related to impact excitation is impact ionization and its inverse process, three-body recombination. Impact ionization is impact excitation, but when the incident free electron has enough kinetic energy that when it strikes the ion, as opposed to moving the bound electron from a lower state $q$ to an upper state $\mathrm{p}$, it cause the bound electron to become a free electron with some new kinetic energy, $E_{\text {kinetic } 2}$ (figure 1.6). In reverse, this is known as three-body recombination, because there must be two electrons within the ion sphere that must collide (12), leaving one with more kinetic energy and the other with a loss in energy that allows the ion to capture it.

As impact ionization and three-body reconnection are inverse processes, they 


\subsection{Spectroscopy as a means to document Atomic Kinetics}
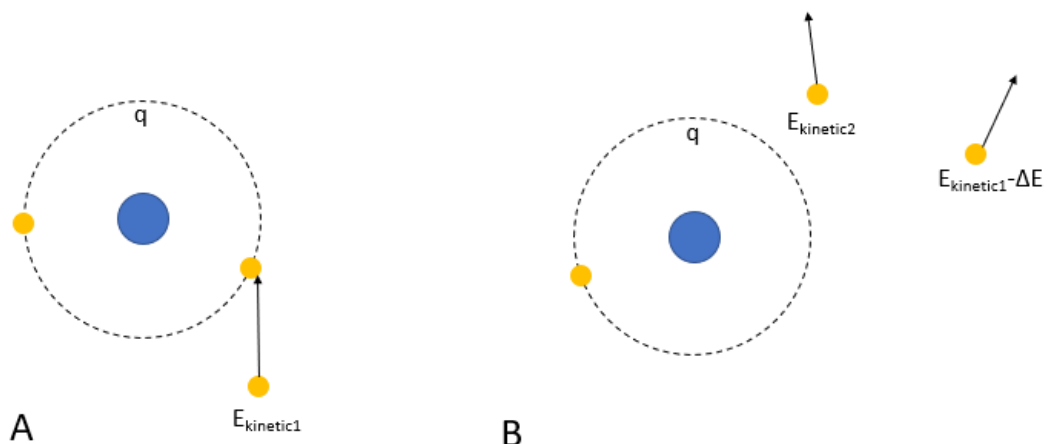

B

Figure 1.6: Impact Ionization: (A) An electron with some energy $E_{k i n e t i c}$ collides with an ion or neutral atom in state q. (B) This transfers some of the energy to a bound electron, allowing it to exit the ion or neutral atom, creating a more highly ionized ion, while the free electron now has a loss of energy, where $\Delta E$ is equal to the energy lost to the now free electron.

can be related to each other, provided that the system is in LTE, using

$$
\frac{S(q, Z)}{R_{3}(q, Z+1)}=2\left(\frac{m c^{2} T_{e}}{2 \pi(\hbar c)^{2}}\right)^{3 / 2} \frac{g_{q}(Z+1)}{g_{q}(Z)} e^{-\frac{E_{Z+1, q}-E_{Z, q}}{T_{e}}} .
$$

$S(q, Z)$ and $R_{3}(q, Z+1)$ are the impact ionization and three-body recombination rates respectively and $E_{Z, q}$ is the energy of an ion in state q and charge state Z.

Calculating $S(q, Z)$ is difficult, much like impact excitation this quantity depends on the electron distribution function, but it also depends on it for two electrons as opposed to just one. The complications of impact ionization lead to many different models to represent this in plasma calculations. The most general model we could find was one by Baronova for Fujimoto (9), which is also based off of the Born-Coulomb assumption, given by

$$
\sigma_{q, c}=\frac{4 \pi a_{o}^{2}}{Z^{4}}(q)^{\frac{11}{4}} f_{q, c} \ln \left(\frac{E_{\text {kinetic } 1} q^{2}}{Z^{2} E_{R}}\right) \frac{Z^{2} E_{R}}{E_{\text {kinetic } 1} q^{2}},
$$

where $\sigma_{q, c}$ is the impact ionization cross-section, $q$ is the principle quantum number for the initial state, $f_{q, c}$ is the oscillator strength for going from the state $q$ to the continuum and $E_{\text {kinetic1 }}$ is the kinetic energy of the initial free electron. By 
integrating this with velocity over the electron energy distribution function the impact ionization rate can be found,

$$
S(q, Z)=\frac{16 \pi a_{o}^{2} E_{R}}{Z^{2}}\left(2 \pi m T_{e}\right)^{-1 / 2}(q)^{7 / 4} f_{q, c} e^{-\frac{Z^{2} E_{R}}{p^{2} T_{e}}} .
$$

The impact ionization rate then needs to be calculated for every state $q$ and charge state for each ion. It should be noted that this only applies when $\frac{E_{k i n e t i c 1} p^{2}}{Z^{2} E_{R}}>>1$. In order to perform the integration over the electron energy distribution function, the assumption that $\ln \left(\frac{E_{\text {kinetic }} q^{2}}{Z^{2} E_{R}}\right)=1$ was used. This implies that while this model can work over a wide range of ions, it can often leave out a sizable amount of ionization.

A less general group of models was put forth by Younger (29) who used a Distortion wave approximation. The Younger models cover Hydrogen-like through Beryllium-like, which are the most applicable charge states to our experiments. The model is parametrized as

$$
\begin{array}{r}
\sigma_{q, c}=\frac{T_{e}}{\left|E_{i}(Z, q)\right|^{3}}\left(A\left(1-\frac{E_{i}(Z, q)}{E_{\text {kinetic }}}\right)+B\left(1-\frac{E_{i}(Z, q)}{E_{\text {kinetic }}}\right)^{2}+\right. \\
\left.C \ln \left(\frac{E_{\text {kinetic }}}{E_{i}(Z, q)}\right)+D \frac{E_{i}(Z, q) \ln \left(\frac{E_{\text {kinetic }}}{E_{i}(Z, q)}\right)}{E_{\text {kinetic }}}\right) .
\end{array}
$$

Here $E_{i}(A, q)$ is used as the ionization energy for an ion with charge state Z in state q, similar to how $E_{R}$ is used as the ionization energy for Hydrogen. $A, B, C, D$ are all constant from the parametrization to an inverse power law, with $A$ and $B$ given by

$$
\begin{aligned}
& A=\sum_{0}^{3} \frac{a_{i}}{\left(Z-Z_{B}+1\right)^{i}}, \\
& B=\sum_{0}^{3} \frac{b_{i}}{\left(Z-Z_{B}+1\right)^{i}},
\end{aligned}
$$

where $Z_{B}$ is the number of bound electrons for that ion. The values of $C, D, a_{i}$ and $b_{i}$ are given in Younger's papers and are too many to list here (29), (30) and (31). The rates can be obtained by integrating the electron energy distribution 


\subsection{Spectroscopy as a means to document Atomic Kinetics}

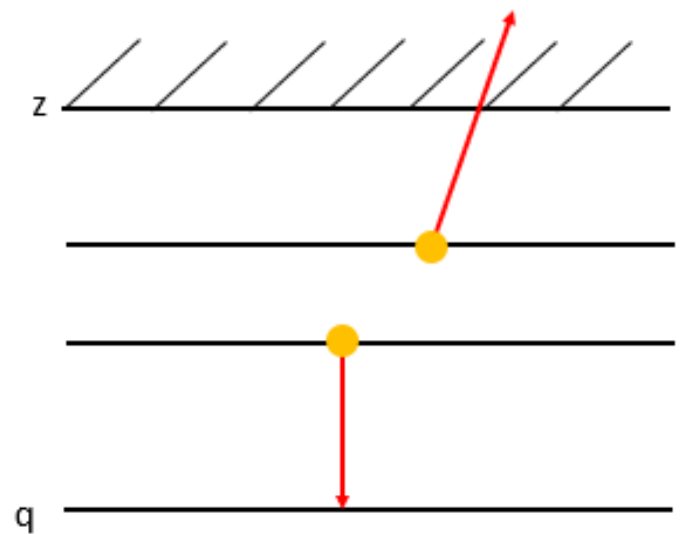

Figure 1.7: Autoionization occurs when two electrons in an ion are both in excited states. When one decays to a lower energy state the excess energy goes into the remaining excited electron as opposed to producing a photon. The excited electron then becomes a free electron, leaving the ion.

function (EEDF), or found in Younger's papers. It should be noted that these models match more detailed models well, and so can be used (13).

\subsubsection{Autoionization and Dielectric Recombination}

The last rate coefficients that need to be calculated are those for autoionization and dielectric recombination. It is important to note that these processes only occur in non-Hydrogen plasmas, as they require two or more electrons to occur. Autoionization is the process in which an ion has two or more electrons in excited states, and when one decays to a lower state, the energy that would normally be emitted as a photon instead is transferred to the other electron. This energy allows the electron to transition to one of the continuum states, thus further ionizing the ion (figure 1.7).

Dielectric recombination is a two step process that occurs when a free electron is captured by an ion. The energy gained by the capture is transferred to a bound electron in a lower state, causing it to transition to a higher state. This is similar to radiative recombination, but instead of emitting a photon, the energy goes to a bound electron. The second step is that the electron now in an excited state decays back down to a lower state, emitting a photon (figure 1.8). 


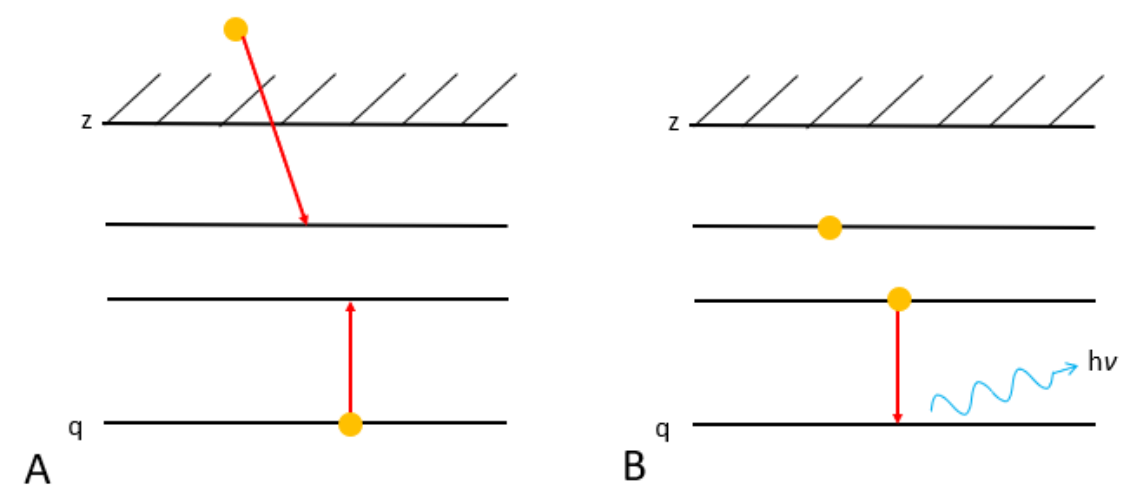

Figure 1.8: Dielectric Recombination: (A) An electron in the ion sphere is captured by the ion. The energy from the capture excites a lower state electron, leading to two electrons in an excited state. (B) One of the electrons decays back to a lower state, emitting a photon. Through this process the ion reduces its charge state.

It should be noted that autoionization is the inverse process of the first step of dielectric recombination. Another note is that unlike other processes involving free electrons, the capturing process (first part of dielectric recombination) is a resonant process, meaning that it can only happen with electrons in a certain energy range, $\delta E$, as opposed to any energy.

Due to dielectric recombination being a two step process, while autoionization is not, it becomes apparent that their cross-sections and rates are not simply equal to each other, as they will have different units. The rates can be related by

$$
n_{e} n_{z+1} C_{z+1, p^{*}}=n_{z} A_{p^{*}}
$$

where $C_{z+1, p^{*}}$ is the rate coefficient for dielectric recombination and $A_{p^{*}}$ is the rate of autoionization. This makes sense as dielectronic capture depends on the density of electrons $\left(n_{e}\right)$ as well as the density of ions in charge state $z+1$, while autoionization only depends on the density of ions in charge state $z$.

Autoionization is very similar to radiative decay, thus its rate coefficient is often written as an Einstein coefficient $\left(A_{p^{*}}\right)$, which represents the rate at which a particle in state $\mathrm{p}$ will decay and ionize a neighboring bound electron. There is no simple analytical formula for autoionization (13) and the same holds true for 
dielectric recombination. This is an active area of research, thus there are many papers trying to solve the problem (32), (33), (34), (35). 
1. INTRODUCTION 
2

\section{Experiments}

Physics is hard.

-Igor Golovkin

\subsection{The Z Machine and Dynamic Hohlraum}

The experiments performed for this dissertation used Sandia National Lab's Z Machine located in Albuquerque NM. The Z Machine (36) is currently the world's most powerful z-pinch (37). Z uses 36 Marx banks (38) to hold and discharge up to $85 \mathrm{kV}$. When this is discharged, it produces $26 \mathrm{MA}$ of current that flows to the load in $100 \mathrm{~ns}$, with a peak current lasting for 3 ns. For these experiments, the load was the Z Pinch Dynamic Hohlraum (ZPDH) (39), which consists of two tungsten wire arrays, an inner array with 120 wires and an outer array of 240 wires and a core of $\mathrm{CH}_{2}$ foam ( figures 2.1 and 2.2).

Images of the pinch taken using a multilayer mirror (MLM) have the pinch stagnation size as approximately $1.5 \mathrm{~mm}$. The ZPDH can produce up to $220 \mathrm{TW}$ of x-ray power, and 1.6 MJ of total irradiated energy in an implosion, making it the world's most powerful source of broadband x-rays. The ZPDH has been used in many experiments at Sandia National Labs (42) (43), and is very reproducible as far as peak power and intensity, figure 2.3 shows this as a twenty shot average.

An aspect of the pinch that was not not yet mentioned is the possibility of reradiation during the pinch implosion. At the start of the implosion, photons will 


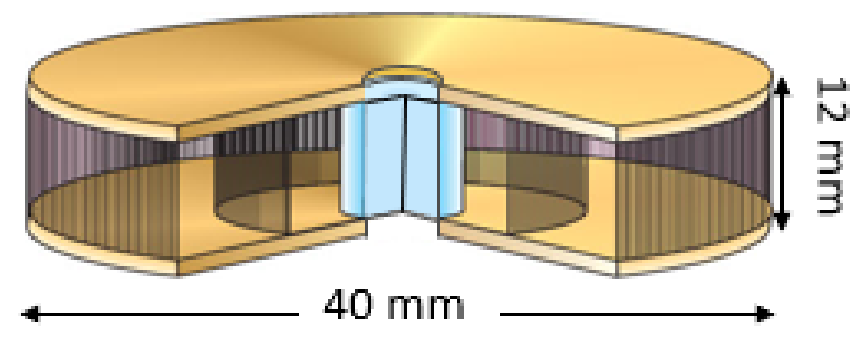

Figure 2.1: The ZPDH consists of two nested tungsten wire arrays, with an initial radius of $2 \mathrm{~cm}$ and a $\Delta L$ of $1.2 \mathrm{~cm}$. Modified from (40)

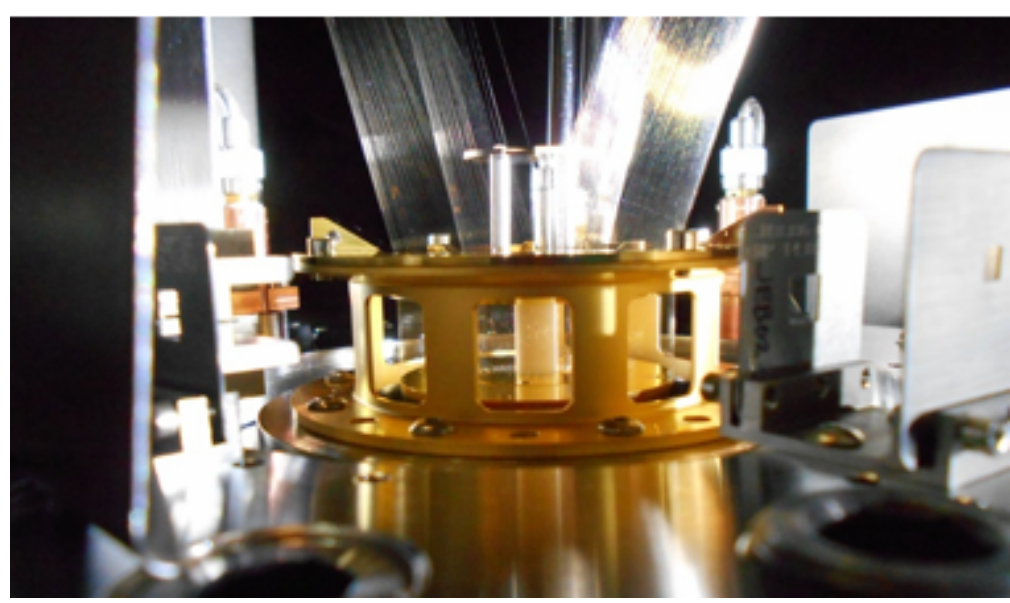

Figure 2.2: A Picture of the load hardware, including the ZPDH before a shot is fired. The gold colored cylinder surrounding the wires is the Return Current Can (RCC). The holes in the RCC are lines of sight (LOS) which allow for diagnostic access to the pinch. (41) 
Radial X-ray Power and Energy (20 shot average)

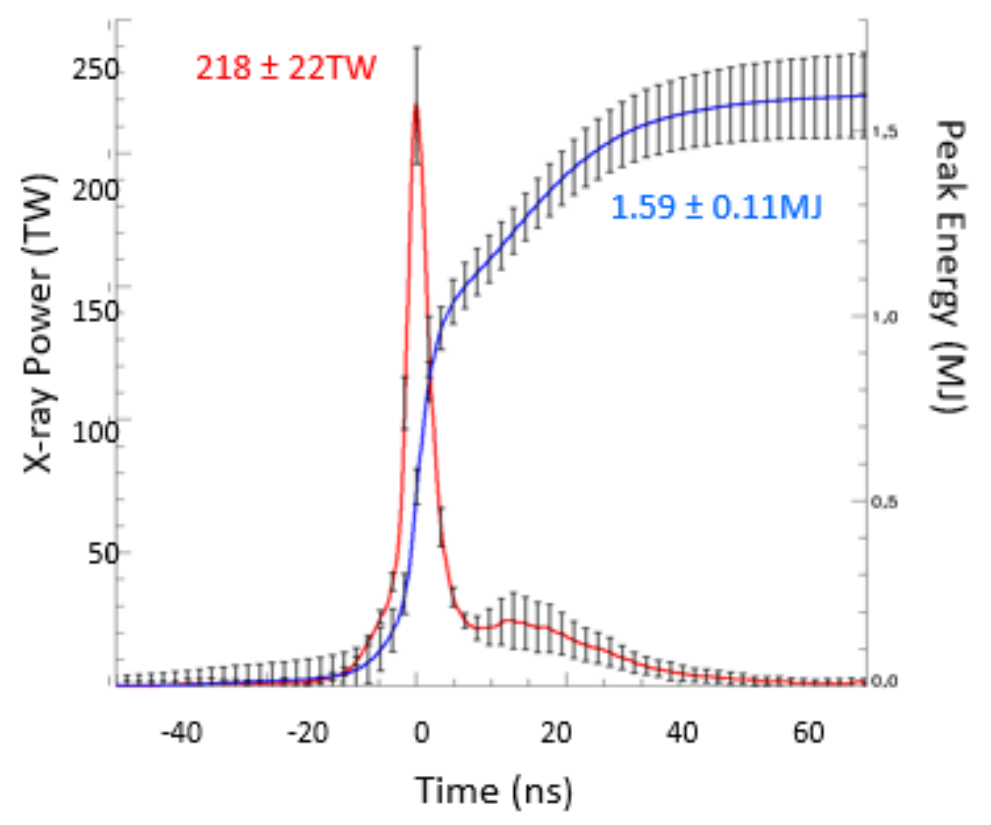

Figure 2.3: The highly reproducible nature of the $\mathrm{ZPDH}$ is shown both in $\mathrm{x}$-ray power (red) and in total energy (blue). The x-ray peak is approximately $3 \mathrm{~ns}$ in width. Modified from (40) 


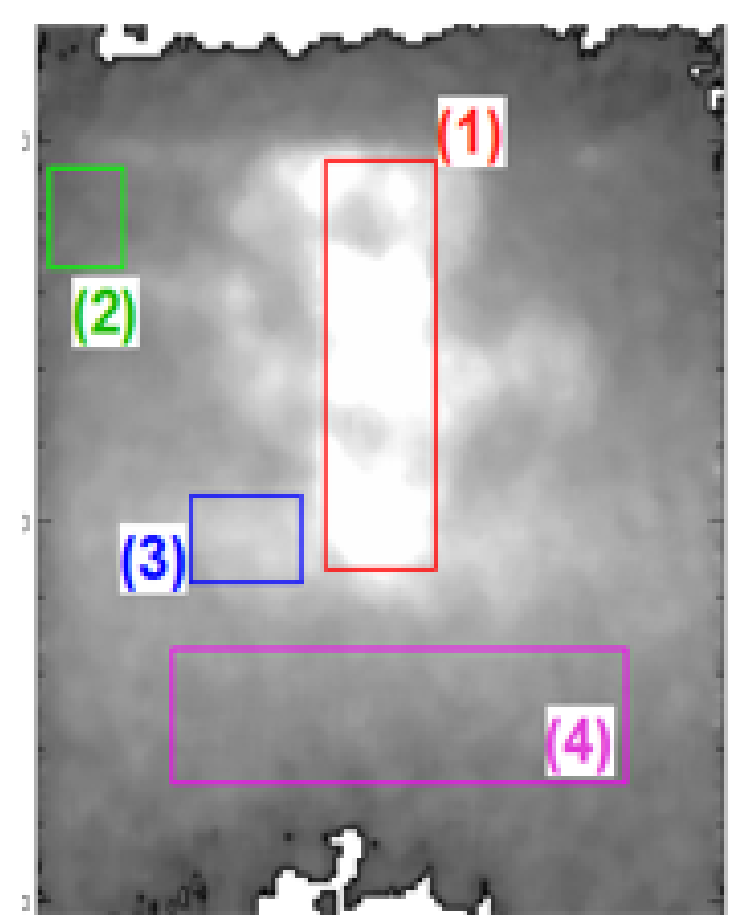

Figure 2.4: An MLM image from a ZPDH shot. The radiation has been broken into four aspects. 1. The pinch itself which is the hottest section. 2. The RCC wall, which is the coldest section and often left out of calculations for the total radiation. 3. The inner bottom glide, which is the section of floor closest to the pinch. 4 . The outer bottom glide, which makes up the floor further from the pinch.(46)

strike the surrounding hardware, heating them up. This hardware can then emit its own characteristic radiation. This means that the actual radiation from the pinch is not a simple blackbody curve, but multiple blackbody curves overlayed. G. Loisel currently has a model that best fits the MLM data from multiple ZPDH shots (44) which uses three blackbodies in total, the pinch itself, the "inner glide" and the "outer glide". These latter two are annuli which represent the floor of the pinch; this can be seen in figure 2.4. Fortunately, this re-radiation has so far been seen to have minimal effect on our experiments (45).

Due to the ZPDH being so powerful and so reproducible, it is an excellent platform for performing HED physics experiments. Experiments performed for this dissertation were done as part of the Z Astrophysical Plasma Properties (ZAPP) collaboration, which is a collaboration between three universities (University of 


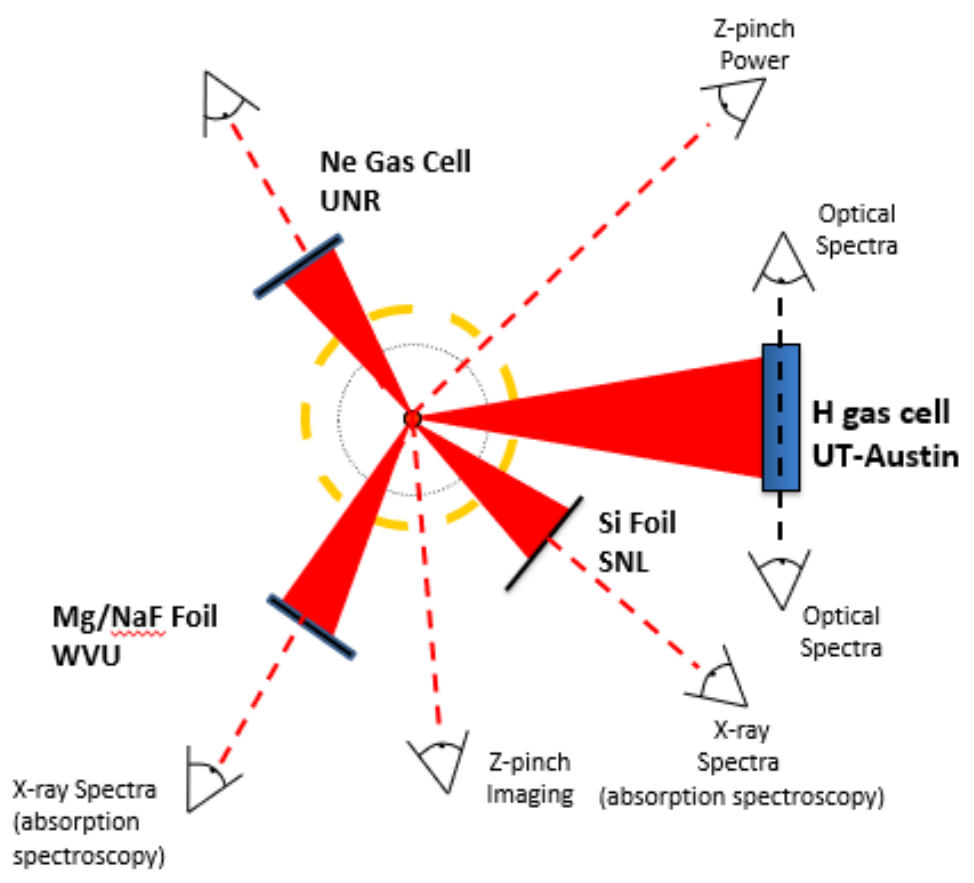

Figure 2.5: The arrangement of the lines of sight for a typical ZAPP shot. WVU uses LOS 130 to collect time-integrated spectra of a MgNaF foil, UT-Austin collects spectra from a gas cell containing hydrogen or helium and UNR collects timegated spectra from a gas cell containing Ne. Sandia National Labs also fields an experiment looking at spectra from a Si foil.

Nevada-Reno, University of Texas-Austin and West Virginia University) and a national lab (Sandia National Lab). This collaboration allowed for more shots on $\mathrm{Z}$ than any university trying on its own, with the small downside that each group only uses one line of sight (LOS) on the ZPDH (40). There are nine total radially located LOSs on the ZPDH, each with a specific diagnostic that can be fielded there (figure 2.5); for these experiments it was LOS 130, which houses the Time Integrated Crystal Spectrometer (TIXTL).

\subsection{TIXTL}

The Time Integrated Crystal Spectrometer (TIXTL)(47) is ; a crystal spectrometer that is not time-gated. Because it is not time-gated, the spectra it collects 


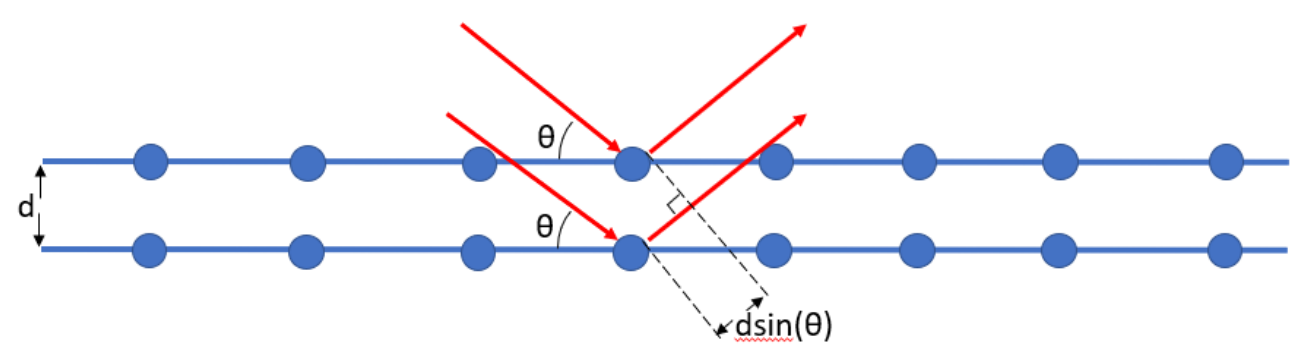

Figure 2.6: Photons (red) can scatter off of particles within the lattice structure of the crystal. The difference in path length between the two is $2 d \sin (\theta)$, and if this distance is equal to an integer multiplication of the wavelength, then there will be one hundred percent constructive interference.

are collected over the entire pinch event. This is mitigated by the fact that the majority of the x-rays come during the peak intensity of 3 ns. The TIXTL uses a cylindrically bent crystal as its means for dispersion, allowing for spectral resolution along one axis and spatial resolution along the other. In general, an x-ray crystal works via Bragg diffraction.

Bragg diffraction of coherent radiation is a phenomena common in crystals due to their lattice structure. When x-rays strike the crystal, photons of the same phase scatter from different points in the lattice structure. There is constructive interference so long as the difference in path length (related to distance between lattice points) is equal to an integer multiplied by the light's wavelength. This can be seen with the equation

$$
n \lambda=2 d \sin (\theta) .
$$

Here $\lambda$ is the wavelength of the light, $\mathrm{d}$ is the inter-lattice spacing normal to the face of the crystal, $\mathrm{n}$ is an integer and $\theta$ is the reflection angle. This effect can also be seen in figure 2.6 .

It should be noted that the crystals used in the TIXTL are cylindrically bent. This cylindrical bending allows for light of the samewavelength to disperse from two spatially separated points on the crystal and still produce meet at the same point on the film. This is due to the curve in the crystal creating different angles at different points ( figure 2.7). 


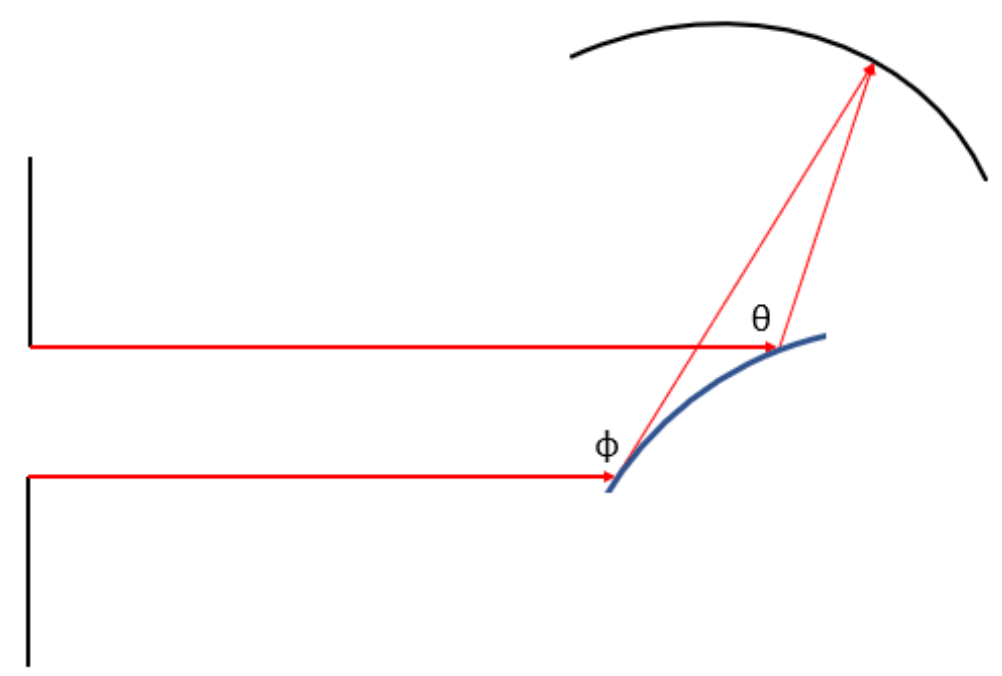

Figure 2.7: Photons (red) from different originating points but the same wavelength, will strike the crystal (blue) with different incident angles, resulting in different reflected angles $(\theta$ and $\phi)$. Due to this the light will defract to the same location on the film (black).

In the experiments discussed here, two types of crystals were used, a Potassium Acid Phthalate (KAP) and a Thallium Acid Phthalate (TAP). The KAP crystals were used in first order, meaning $n=1$ in equation 2.1, whereas the TAP crystals were used in second order, so $n=2$ in equation 2.1. Second order has half of the inter-lattice spacing that first order diffraction has, but second order is much less bright than first order. This is just like optical diffraction, the first spot is always the brightest. This is why we chose to use a TAP as opposed to a KAP in second order, a TAP has a much higher reflectivity. The crystal information is detailed in table 2.1 .

When fielding the TIXTL, the crystals can be mounted two different ways depending on what spatial resolution is desired; axially resolving or radially resolving. Axially resolving means that the spectrometer spatially integrates the signal along the width of the pinch, but can still resolve along the axis of the pinch. The source size for an axially resolved shot is the width of the pinch at stagnation, which is $1.5 \mathrm{~mm}$, due to the radial axis being the axis lacking spatial resolution. Radially resolved shots have the crystal set up perpendicular to how an axially shot would be, such that the source is spatially integrated axially and 


\begin{tabular}{|c|c|c|}
\hline Crystal & Order of Diffraction (n) & Inter-lattice Spacing (d) \\
\hline $\mathrm{KAP}$ & 1 & $26.63 \AA$ \\
\hline TAP & 2 & $12.73 \AA$ \\
\hline
\end{tabular}

Table 2.1: The crystals used in the experiments, along with what order of diffraction was used and the inter-lattice spacing. The KAP crystal has a wavelength range of $7-15 \AA$, and the TAP crystal has a range of $7.3-8.3 \AA$

spatially resolved radially. With a radially resolving crystal, the source size is determined by the limiting aperture used in the shot, which allows for only the central portion of the pinch to be seen. This will be discussed more in the next section.

The crystals used in the TIXTL also contribute to instrument broadening. Due to bending induced imperfections in the atomic structure of the crystal lattice there is a slight broadening of the spectra. This broadening is often referred to as the crystal rocking curve. The crystal rocking curve is measured by placing the crystal at a specific angle with respect to a monochromatic light source, then "rocking" the crystal slightly to see how far the crystal can move before the diffracted light changes position on the film. This experimental procedure is done at Sandia using a Manson source as the monochromatic light source (48).

\subsection{Experiments on the RCC}

For these experiments, the ZPDH was used as a heating source and backlighter for foils composed of $\mathrm{MgO}_{2} \mathrm{NaF}$, and the TIXTL was used as the spectrometer. These foils were glued directly onto the $\mathrm{ZPDH}$, to minimize the distance to the pinch without disturbing the pinch implosion. Previous experiments that we performed showed that the standard fielding position of $4.4 \mathrm{~cm}$ did not achieve high enough temperatures. A limiting aperture with size $3 \mathrm{~mm} \times 3 \mathrm{~mm}$ was placed $1.75 \mathrm{~cm}$ behind the foils relative to the pinch. This was done so that only the center $9 \mathrm{~mm}^{2}$ of the foil was used to avoid possible edge effects. Imaging slits were also placed $\frac{3}{4}$ of the way to the TIXTL, which allowed for roughly 3 times 


\subsection{Experiments on the RCC}

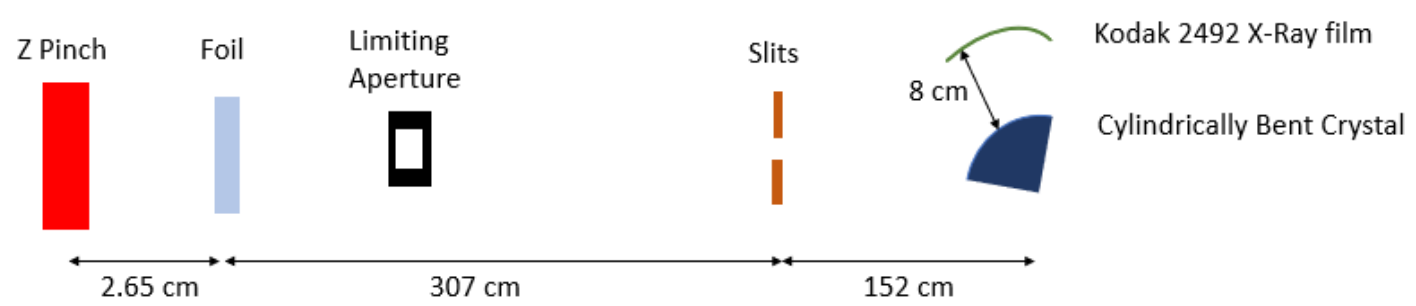

Figure 2.8: The foil used in the experiment is glued to the RCC, $2.65 \mathrm{~cm}$ from the pinch axis. A $3 \times 3 \mathrm{~mm}$ limiting aperture is placed behind the foil to ensure only the center of the foil is seen. Imaging slits are fielded $3 \mathrm{~m}$ from the pinch, these allow for greater spatial resolution as well as a magnification 3. Lastly, the TIXTL is fielded with a cylindrically bent crystal and Kodak RAR2492 x-ray film.

the magnification, as the distance from pinch to slit $(3 \mathrm{~m})$ is three times larger than distance from slit to crystal $(1 \mathrm{~m})$. This shot geometry can be seen in figure 2.8. The diagnostics team at Sandia fielded these experiments for us according to our specifications.

\subsubsection{Foils}

The foils themselves are made of $\mathrm{MgO}_{2} \mathrm{NaF}$ and are $13 \mathrm{~mm} \times 11 \mathrm{~mm}$, as that size allowed for them to cover the LOS viewport on the RCC. Each multi-layered foil was heavily tamped. The elements were chosen due to their proximity to each other on the periodic table and due to their ease of creating a foil using them. The proximity on the periodic table allowed for small differences in wavelength between the three elements' spectral features. Due to this, spectra from the three elements of interest $(M g, N a$ and $F$ ) could be collected in a single shot, and all elements would have similar charge states. The tamper was composed of $\mathrm{C}_{6} \mathrm{H}_{6}$ plastic and the thickness varied across different shots, specifically three different tampers were used, $4 \mu m, 7 \mu m$ and $15 \mu m$ over 10 different $Z$ shots. This is considered "heavily-tamped" as the $\mathrm{MgO}_{2} \mathrm{NaF}$ multi-layered foil (the part we care about) is $0.4 \mu m$, making the tamper at least ten times larger than the foil. The thickness of the foils was chosen such that the transmission through the foil would not saturate (transmission below 0.35 ) while also allowing for high-N transitions to be seen above the noise. The large amount of tamping prevented 


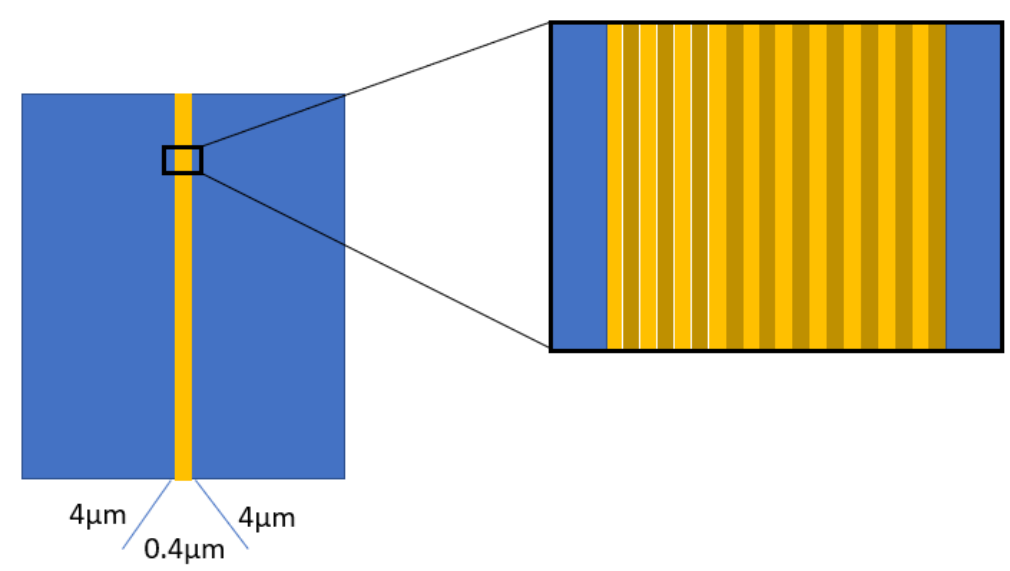

Figure 2.9: A view of a $4 \mu \mathrm{m}$ tamped foil The blue is the $C_{6} H_{6}$ while the yellow is $\mathrm{NaF}$ and the gold is $\mathrm{MgO}_{2}$. The $\mathrm{MgO}_{2}$ and the $\mathrm{NaF}$ are interleaved to prevent stratification within the plasma. It should be noted that the only difference for more highly-tamped samples was the amount of tamper, all $\mathrm{MgO}_{2} \mathrm{NaF}$ foils would come from the same batch.

expansion of the foil during the experiment and succeeded in maintaining LTE conditions. The higher amounts of tamper were later added to further reduce expansion of the foil, allowing for higher densities to be achieved. The foil itself was composed of alternating ten layers of $\mathrm{MgO}_{2}$ and ten layers of $\mathrm{NaF}$ (figure 2.9), as the co-deposition chamber at Raytheon was non-operational at the time of these experiments. Bailey et al (4) has shown that if a foil is comprised of more than 8 alternating layers then enough mixing occurs to prevent non-uniformities through the foil.

Two sets of foils were used during the course of the experiments. While these foils were specified to be identical, the product turned out to be different. These two batches of foils are composed of the same elements with the same amount of tamper-thickness, but the stoichiometry varies slightly. Table 2.2 shows the different areal densities of elements found within the two sets of foils. These values were determined using Rutherford backscattering (RBS) spectra. 


\begin{tabular}{l|l|l|l|l|l} 
Foil sample & $\boldsymbol{N a}\left(\mathrm{cm}^{-2}\right)$ & $\boldsymbol{F}\left(\mathrm{cm}^{-2}\right)$ & $\boldsymbol{M g}\left(\mathrm{cm}^{-2}\right)$ & $\boldsymbol{O}\left(\mathrm{cm}^{-2}\right)$ & Thickness $(\AA)$ \\
\hline & \multicolumn{4}{|l}{} \\
$\mathrm{A}$ & $6.13 \times 10^{17}$ & $6.74 \times 10^{17}$ & $6.74 \times 10^{17}$ & $5 \times 10^{17}$ & 3494 \\
$\mathrm{~B}$ & $6.3 \times 10^{17}$ & $6.3 \times 10^{17}$ & $5.9 \times 10^{17}$ & $1.4 \times 10^{18}$ & 3011
\end{tabular}

Table 2.2: The RBS measurements for the two sets of foils used in the experiments.The errors for these measurements are approximately ten percent.

\subsubsection{Z Shots}

As stated there were three different foil tamper thicknesses fielded for these experiments. This data was collected over the course of three years (Appendix A), as the $\mathrm{Z}$ machine can only fire once a day and ZAPP receives about two weeks per year for experiments. Fortunately, the TIXTL is comprised of two arms, meaning two sets of spectra can be collected per Z shot. However, a Z shot is an intensely chaotic and destructive environment, and some shots only have data from one arm of the TIXTL. Overall there were 5 use-able spectra collected from $4 \mu m$ tamped foils, 7 spectra collected from the $7 \mu m$ tamped foils and 3 spectra from the $15 \mu \mathrm{m}$ tamped foils. The shots, along with crystal and foil information are all cataloged in table 2.3

Spectra taken using a KAP crystal typically had a broad spectral range, from $7 \AA$ to $15 \AA$, which allowed for the $\mathrm{Mg}, \mathrm{Na}$ and $\mathrm{F}$ spectral features to be collected with one spectrometer. The absence of Oxygen spectra is simply due to the fact that we did not originally intend for there to be any Oxygen in our foils, but the $\mathrm{Mg}$ oxidized. The TAP crystal has a significantly smaller spectral range, with a range of $7 \AA$ to $8.5 \AA$. After a shot, the film is digitized (figures 2.10 and 2.11).

\subsubsection{Absorption Criterion}

It is important to note that we will be doing absorption spectroscopy measurements of our plasma, and we need to be sure that self emission is limited so as to not affect the measurements. This is because in any absorption measurement, 


\begin{tabular}{l|l|l|l|l|l} 
Shot & Foil type & Tamper & Crystal & R. Direction & Imaging Slits \\
\hline z2950 & A & $4 \mu m$ & 4" KAP & Axial & 300 and $600 \mu m$ \\
z2971 & A & $4 \mu m$ & 4" KAP & Axial & 500 and $500 \mu m$ \\
z3053 & A & $15 \mu m$ & 4" KAP & Radial & 100 and $300 \mu m$ \\
z3141-L & A & $15 \mu m$ & 4" KAP & Radial & 100 and $300 \mu m$ \\
z3141-R & A & $15 \mu m$ & 4" KAP & Radial & 100 and $300 \mu m$ \\
z3194-L & A & $7 \mu m$ & 4" KAP & Radial & 100 and $300 \mu m$ \\
z3194-R & A & $7 \mu m$ & 4" KAP & Radial & 100 and $300 \mu m$ \\
z3275 & B & $4 \mu m$ & 6" TAP & Axial & 500,500 and $500 \mu m$ \\
z3276 & B & $7 \mu m$ & 4" KAP & Axial & 500 and $500 \mu m$ \\
z3286-L & B & $7 \mu m$ & 4" KAP & Axial & 500 and $500 \mu m$ \\
z3286-R & B & $7 \mu m$ & 6" TAP & Axial & 500,500 and $500 \mu m$ \\
z3364-L & B & $4 \mu m$ & 4" KAP & Axial & 500 and $500 \mu m$ \\
z3364-R & B & $4 \mu m$ & 6" TAP & Axial & 500,500 and $500 \mu m$ \\
z3365 & B & $7 \mu m$ & 6" TAP & Axial & 500,500 and $500 \mu m$
\end{tabular}

Table 2.3: The list of shots on $\mathrm{Z}$ that yielded valuable spectra. Not all shots have a distinction between the left or right side of the TIXTL as one side did not have high quality data. The imaging slits came in several styles, all with a minimum of two slits, some with three. R. Direction stands for Resolving Direction, whether or not the axial or radial direction on the pinch had spatial resolution. 


\subsection{Experiments on the RCC}

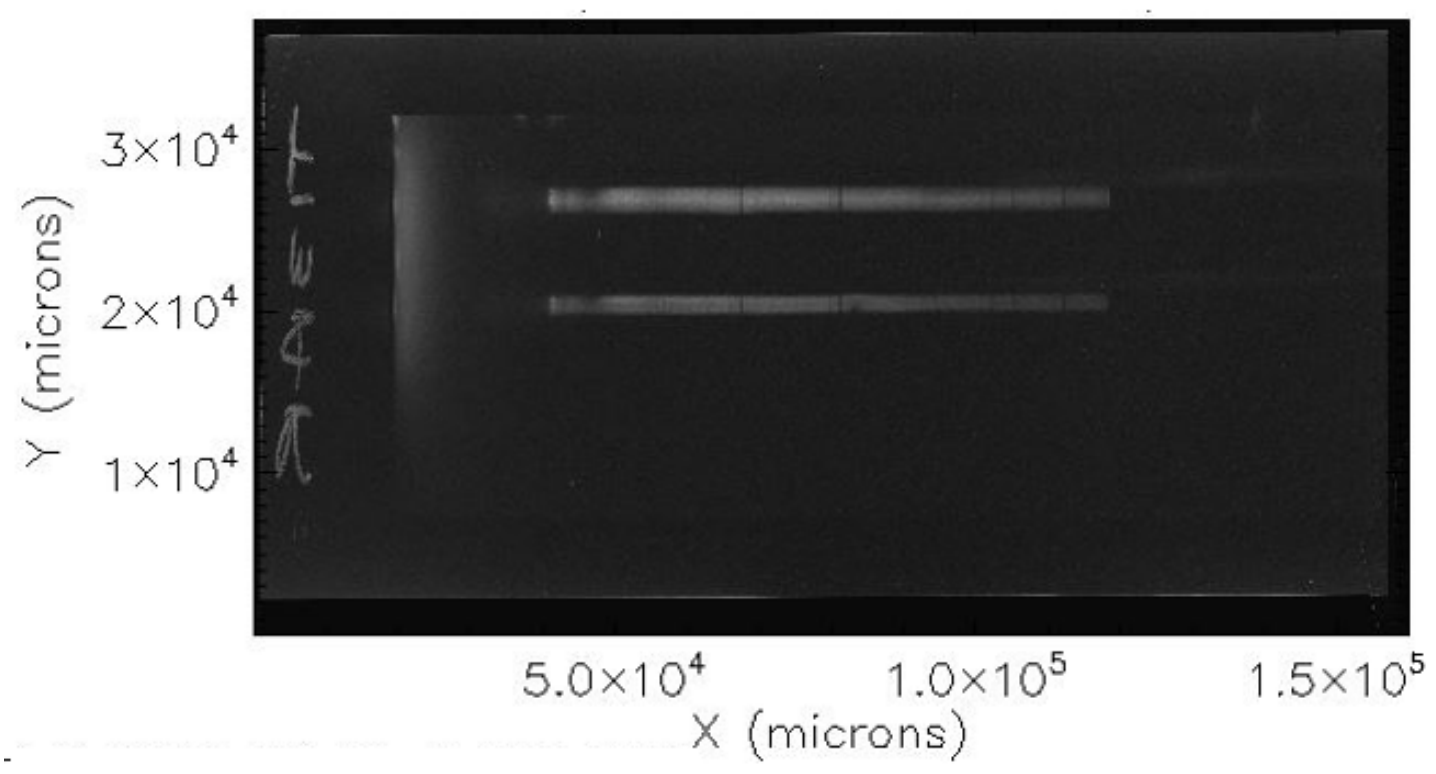

Figure 2.10: Digitized film from shot z 2950 which used a KAP. The Na $\alpha$ lines are in the center of the film. The two slit images are clearly visible here.

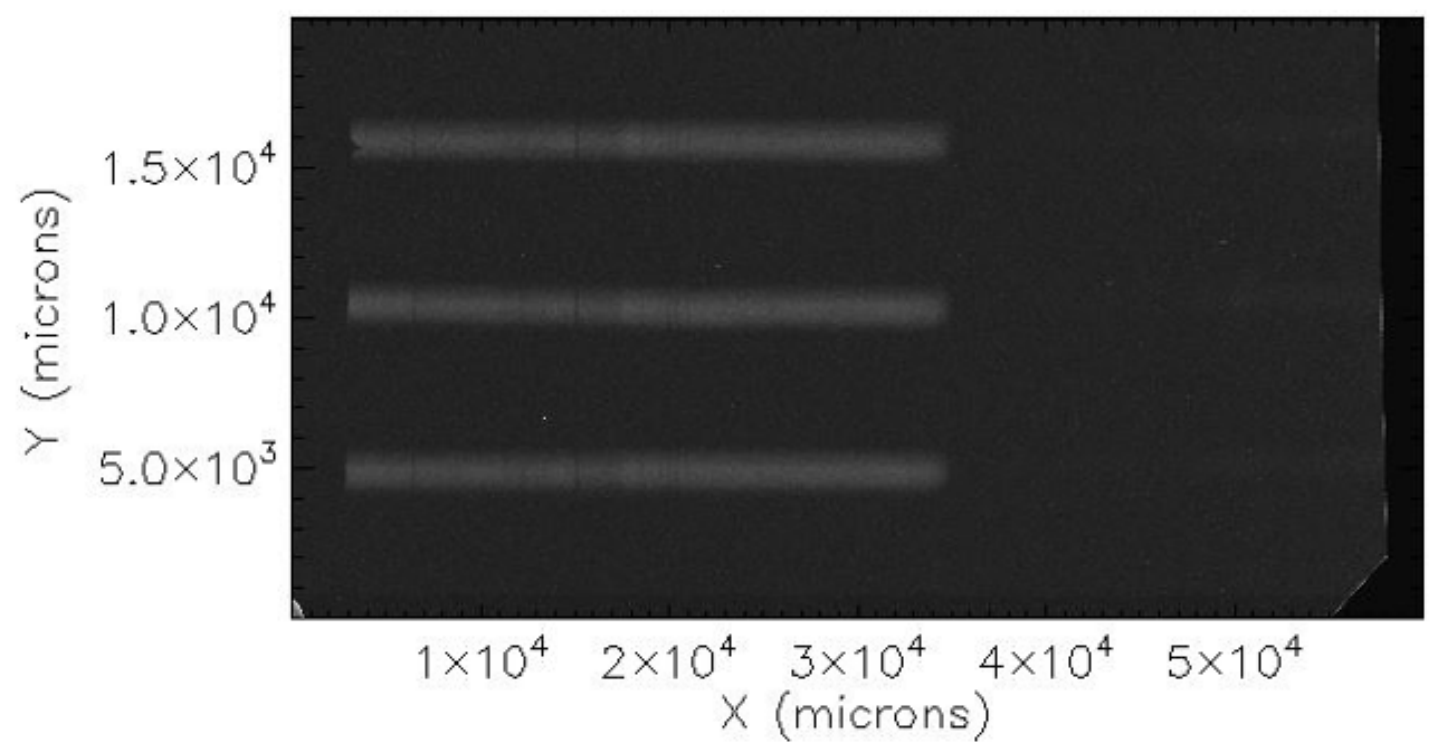

Figure 2.11: Digitized film from z3364-R, which used a TAP crystal. There are fewer spectral features visible due to the decrease in spectral range. The lines that are visible are He-like Mg. This shot featured 3 imaging slits all of the same size. 


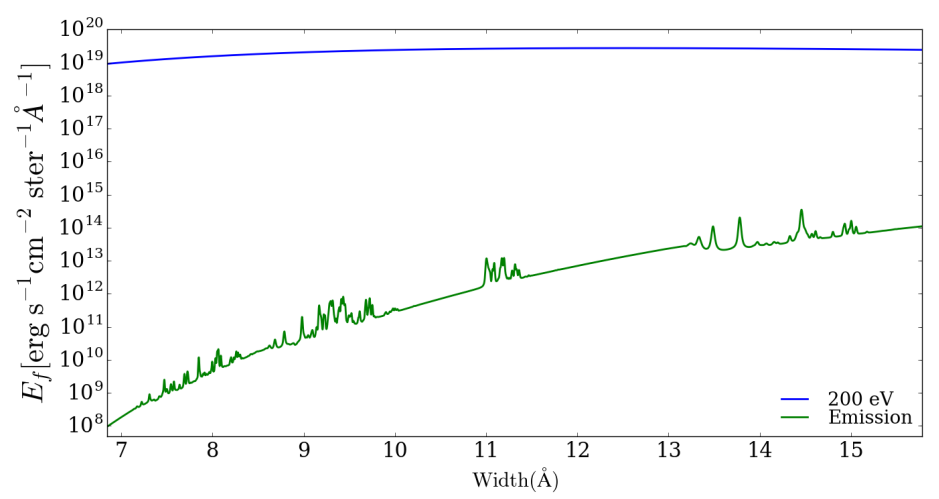

Figure 2.12: Predicted emission from a $54 \mathrm{eV} \mathrm{MgO}_{2} \mathrm{NaF}$ plasma in our spectral range compared with a $200 \mathrm{eV}$ blackbody. It should be noted that even with a lower temperature blackbody, the backlighter is orders of magnitude larger than the self-emission from the plasma.

the intensity measured on the film goes as:

$$
I_{\text {measured }}=B T_{\text {plasma }}+I_{\text {plasma }},
$$

where $I_{\text {plasma }}$ is the plasma's self-emission in intensity units, $B$ is the backlighter profile, and $T_{\text {plasma }}$ is the transmission through the plasma. In order for our results to be trustworthy, we must ensure that $B>I_{\text {plasma }}$. This can be tested by assuming $B$ is Blackbody radiation from the pinch, and comparing this to the intensity from plasma self-emission generated by PrismSPECT. In figure 2.12 we see that the plasma emission is significantly smaller than the backlighter intensity, leading us to conclude that self-emission is negligible for these experiments.

\subsubsection{Instrument Broadening}

Up to this point we have mentioned broadening from the crystal and from the source, but have not discussed these further. Before the discussion of Data itself takes place, it is important to fully address the issue of broadening from measurements and not implicit in the plasma. There are three different sources of broadening originating from how this experiments was performed: source size 


\subsection{Experiments on the RCC}

broadening, crystal rocking curve and broadening from the detector (in our case Kodak RAR 2492 x-ray film).

Source size broadening originates from the fact the the pinch itself is not a point light source, but has some spatial extent. Because of this, light from one point on the pinch will have a different trajectory and endpoint than light originating from a different point. This broadening is entirely an effect of geometry, and thus depends on the spatial extent of the source and the distances from source to crystal and crystal to film. Swartz et al (49) discuss this and provide this relation

$$
W=\frac{S}{\sin (\alpha)} \frac{r \sin (\theta)+L_{2}}{r \sin (\theta)+L_{1}}
$$

where $\mathrm{W}$ is the width, $\mathrm{S}$ is the source size, $L_{1}$ and $L_{2}$ are the distances from the source to the crystal and the crystal to the film, $\theta$ is the Bragg angle of the crystal, $\mathrm{r}$ is the radius of curvature and $\alpha$ is the angle between the film plane and the incident radiation. One can use average values for all of these to get an average broadening, or a ray-tracer can determine the distances and angles and th only inputs would be $\mathrm{S}, \mathrm{r}$ and $\theta$. G. Loisel has a ray-tracer for just this purpose. For our experiments, $\mathrm{S}$ is $1.5 \mathrm{~mm}$ for axially resolving measurements and $3 \mathrm{~mm}$ for radially resolving measurements. It should be noted that the shape of the broadening will take the same shape as the source, fortunately the ZPDH is Gaussian upon stagnation.

Detector broadening is broadening caused by the finite size of the Silverbromide particles that make up the film along with the finite steps that the digitizer takes when uploading the film. The trick here is converting the physical extent of the broadening on the film into a wavelength broadening using

$$
\begin{gathered}
\frac{d \lambda}{d x}=D(\lambda) \\
d \lambda_{\text {det }}=\frac{d \lambda}{d x} d x \\
d \lambda_{\text {det }}=D(\lambda) d x,
\end{gathered}
$$




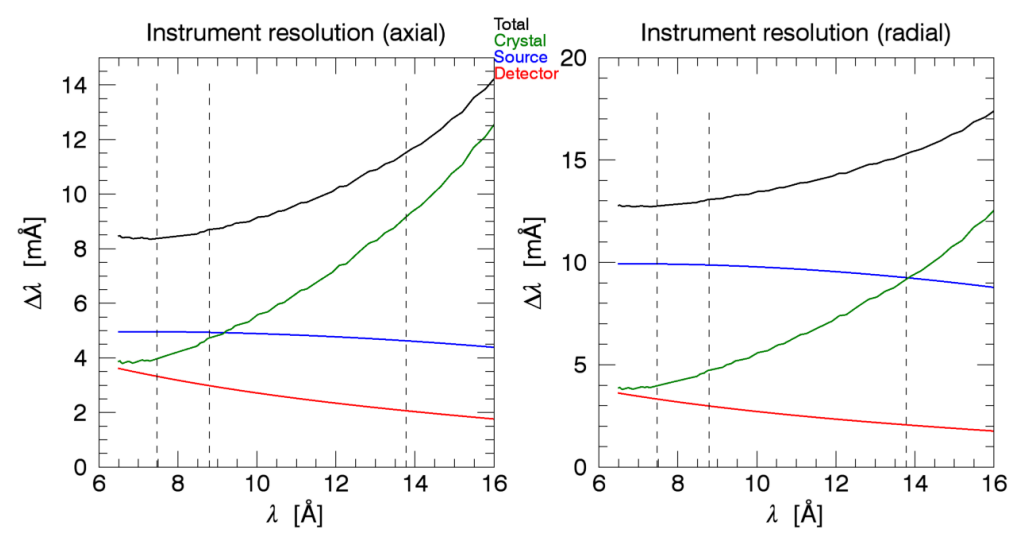

Figure 2.13: The broadening from the three sources plus a total instrument broadening as functions of wavelength for a 4" KAP crystal. The left side is axially resolving and the right side is radially resolving. The three dashed lines are the $M g-H e \gamma, N a-H e \delta$ and the $F-H e \gamma$ lines, which are of interest for both the isoelectronic line ratio study and the Stark broadening study.

here, $D(\lambda)$ is the dispersion relation for the crystal setup (discussed more in the next section), and $\mathrm{dx}$ is the spatial extent of the grain size, which in our case is roughly $2.1 \times 10^{-3} \mathrm{~mm}$. This broadening is also predominantly Gaussian in shape, as it relies on counting statistics to take shape.

The crystal rocking curve was discussed in the last section and the experimental values for our two crystals were provided by G. Loisel and were found to be Lorentzian in shape. This leaves only calculating the total instrumental broadening. Since the broadening constitutes Gaussians and Lorentzians, the total broadening will have the shape of a Voigt profile. As a Voigt profile is very complicated, we chose to use the pseudo-Voigt approximation, which states that the total width of the Voigt is

$$
W_{v}=0.5346 W_{L}+\sqrt{0.2166 W_{L}^{2}+W_{G}^{2}}
$$

This approximation has been found to be accurate to within 0.02 percent so it is reasonable and much quicker than convolving the Lorentzian and the Gaussian to find the width. The widths of all these broadenings can be seen in figures 2.13 and 2.14 . 


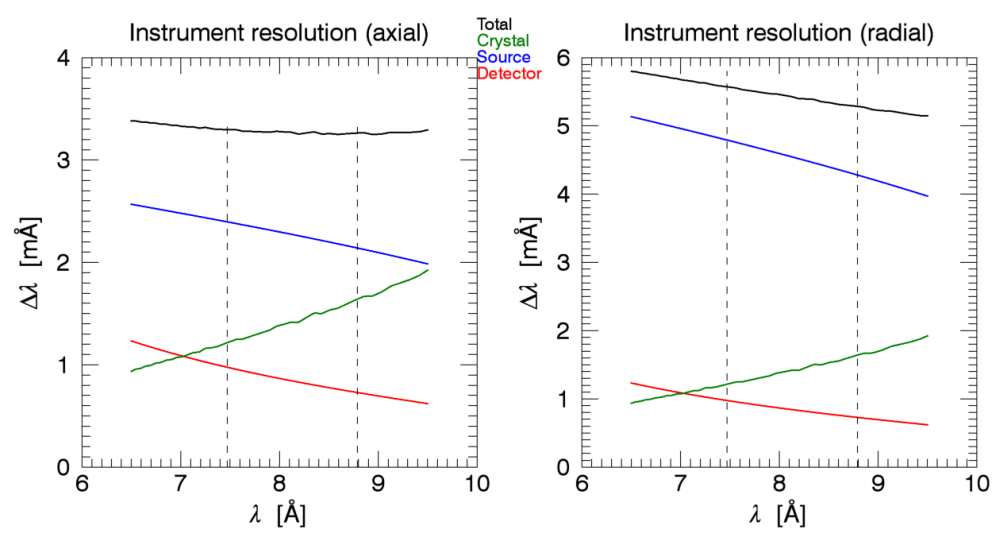

Figure 2.14: The broadening from the three sources plus a total instrument broadening as functions of wavelength for a 6 " TAP crystal in second order. The left side is axially resolving and the right side is radially resolving. The dashed lines represent the $\mathrm{Mg}-\mathrm{He \gamma}$ and the $\mathrm{Na}-\mathrm{He} \delta$ lines. Note how this broadening is much less than that from a KAP in first order.

\subsection{Data Processing}

After the shot is taken, the film is put into a light-tight secure box for transportation to a digitizer, where Linda Nielson-Weber or Antoinette Maestas digitized the film such that it could be processed. Processing the film is the last step before the data can be analyzed. The first step is to check the amount of film fog, as this will alert us to whether the film was saturated from something outside the experiment and allows us to subtract the fog value from the total film exposure $(50)$,

$$
E_{f i l m}=E_{\text {fog }}+E_{\text {absorption }}+E_{\text {emission }}
$$

here $E_{\text {film }}$ is the total film exposure, $E_{f o g}$ is the effect of the fog and $E_{\text {absorption }}$ is the absorption measurement. We take $E_{\text {emission }}$ to be very small, and thus does not affect the measurement. The fog value is determined by taking a wide lineout of a piece of film that was not exposed to the pinch, but was carried with the experimental film the rest of the time. Within the lineout, the film exposure is checked for each pixel, and a Gaussian is fit to this. The peak of the Gaussian is treated as the mean film fog level (figure 2.15). As long as this is below 0.4 


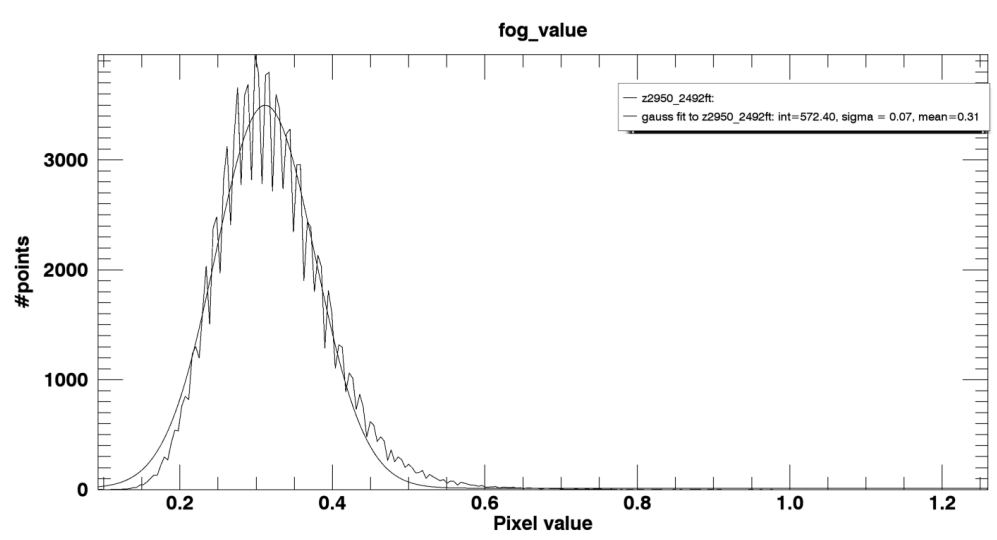

Figure 2.15: The film fog analysis. A lineout if taken on unexposed film, the number of points within the lineout is plotted versus the pixel value of those points and a Gaussian is fit to the distribution. The centroid of the Gaussian is taken as the amount of fog.

then the film was not over-exposed or saturated outside of the experiment and the value can be safely subtracted without involving the non-linear effects of over-exposure.

Then a vertical lineout of the film is taken, so that the centroids of the slits can be found. This is done by determining the points of steepest slope on each side of the slits and extrapolating lines to the slopes (figure 2.16). Where the lines cross is the center of the slit. This allows for the spacing between the slits to be compared on film and to the spacing between the physical slits, allowing for a calculation of the magnification (which is generally around 3).

A single slit is then processed at a time, to be averaged together at the end. A subsection of a slit's spectra is then taken, and for every pixel a horizontal lineout is taken. All of these single-pixel lineouts are then compared to the average lineout to determine if the spectral features shift horizontal location. If the features were to shift horizontally then this could constitute a form of artificial broadening were it not corrected for. The cause of these shifts would be a slight misalignment in the crystal or slits during the experiment, so it is best to check. In general, our spectra is seldom misaligned in this way, as shown in figure 2.17, where the shift-aligned spectra and the original spectra differ by 0.05 degrees.

Next a lineout is taken over the slit region on the film. The width of the 

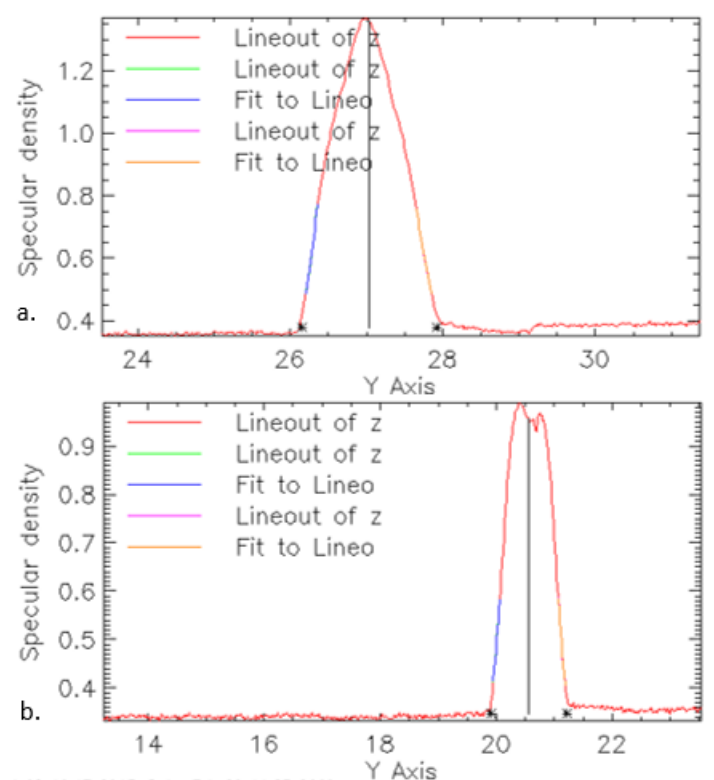

Figure 2.16: The top (a) and bottom (b) slits from shot z2950. The center of the slits is the black vertical line, while the two $*$ denote the edges of the slits.

lineout is determined from the physical spacing of the slits several steps before, this allows for the maximal amount of the slit to be within the lineout, which corresponds to a higher signal to noise. Fiducial lines are then identified from the lineout, so that a conversion from film position to wavelength can take place. The specific lines chosen and how many are chosen depends on the shot parameters, a TAP shot has fewer spectral lines to choose than a KAP. The values used for the fiducial lines are taken from the NIST database. Once the lines are chosen, the spectra can be converted into wavelength space using a fitted polynomial fit (figure 2.18).

Once the film is in wavelength space, lineouts are taken above and below the slit features. This is done to assess the level of exposure that occurs during the implosion, but is not coming from the pinch itself. The two lineouts can be averaged together to avoid any artifacts that may have been present on the film. This average background is then subtracted from the slit spectra, such that only the exposure from the pinch is taken into account.

Once the background has been removed, the filters used on the snout of the TIXTL must be taken into account. The effective filter transmission can be 

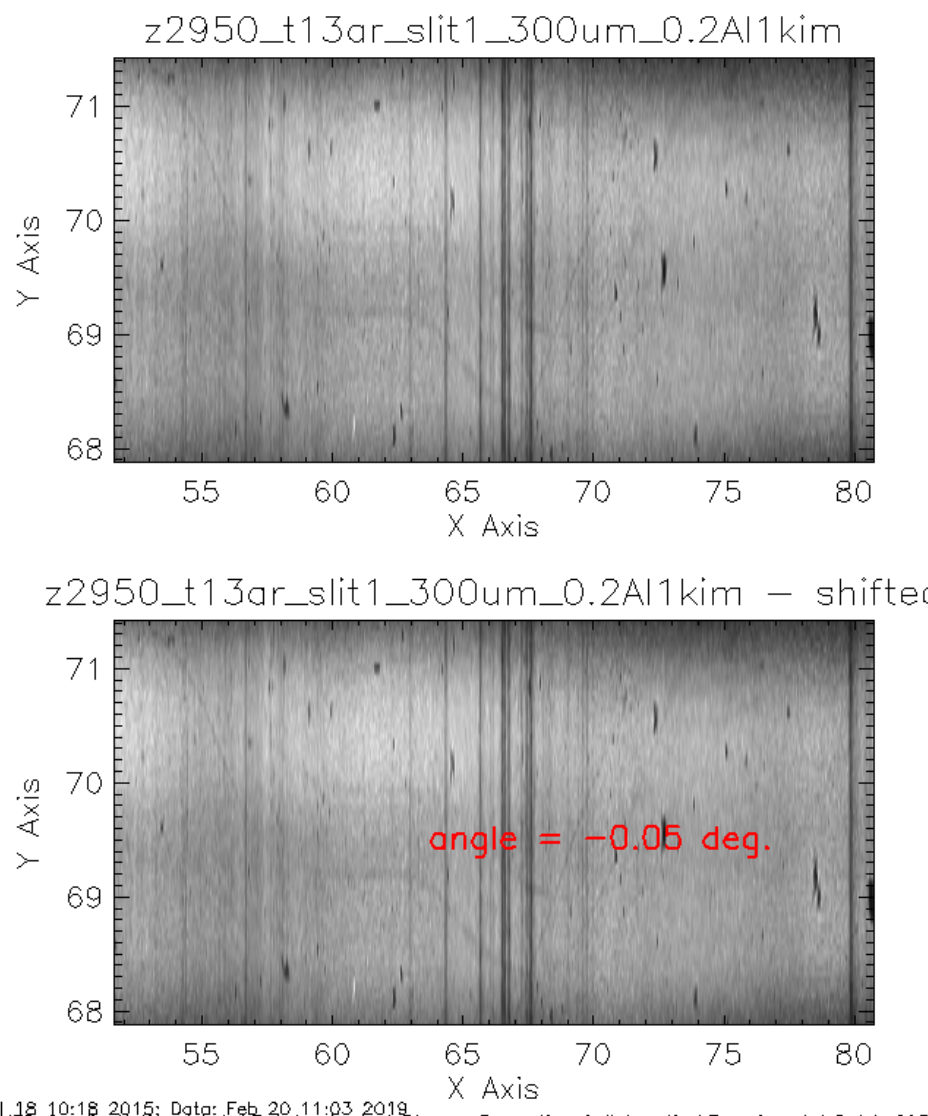

Figure 2.17: The top spectra is the original subset of spectra from z2950's bottom slit, and the bottom is that same subset of spectra but after going through the aligning process. The difference between these two spectra is 0.05 degrees, and is hardly visible to the human eye. 


\subsection{Data Processing}

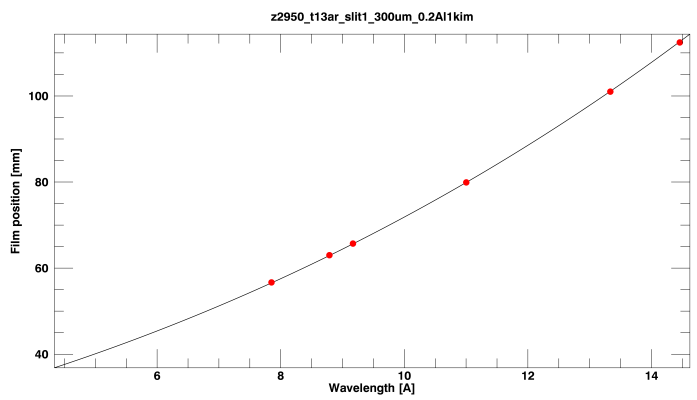

Figure 2.18: The optimized dispersion for z2950's top slit. As this was a KAP shot,the fiducial lines were the $M g-H e \beta, N a-H e \delta, M g-H e \alpha, N a-H e \alpha, F-H e \epsilon$ and $F-H e \beta$. these lines were chosen as the are isolated, easily identifiable and span the range of the spectrometer.

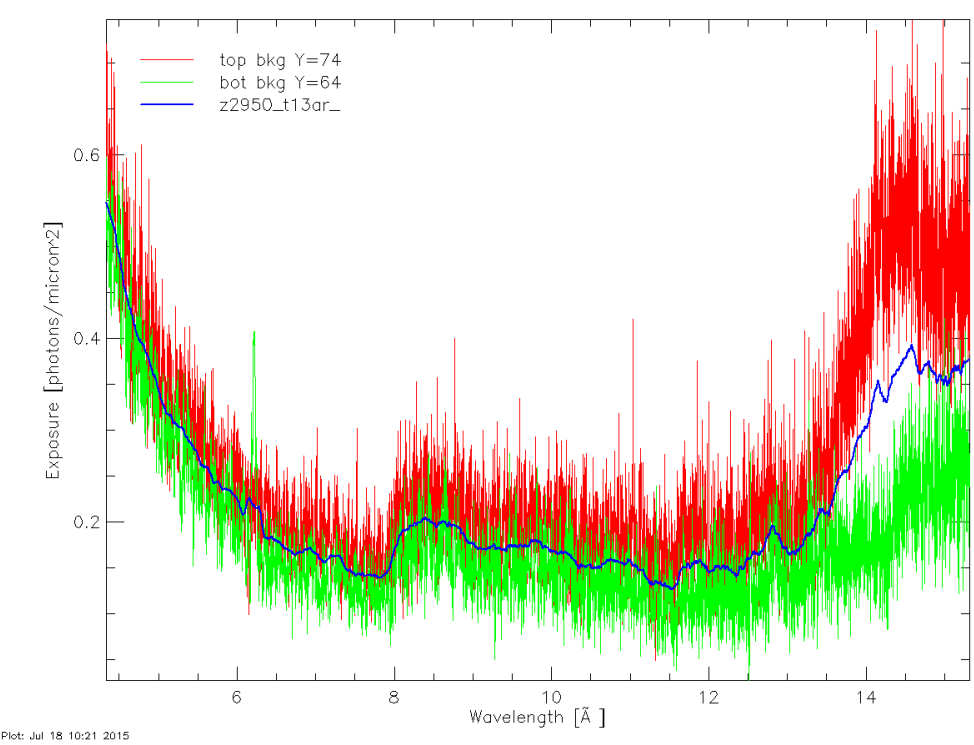

Figure 2.19: The background taken from above the slit features (red) and the background from below(green) are averaged together (blue) for z2950's bottom slit. This is done to avoid any spurious results and average out any artifacts. 


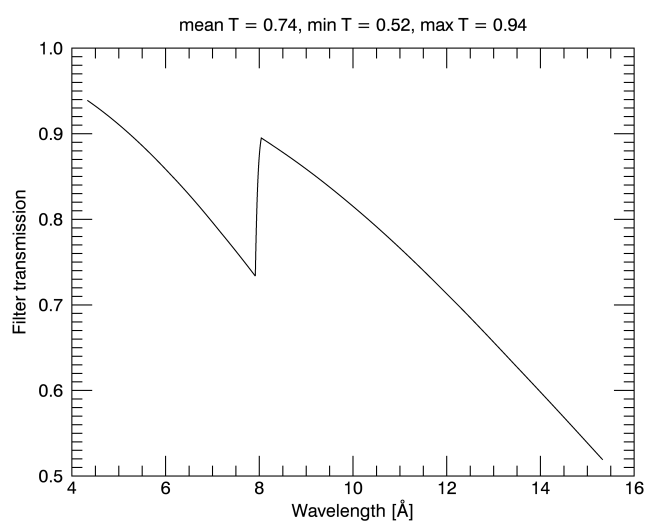

Figure 2.20: The filter transmission for z2950. The filter used for KAP shots is composed of 1 micron of Lexan sandwiched between $150 \mathrm{~nm}$ and $50 \mathrm{~nm}$ of Aluminum, resulting in transmission through $0.2 \mu \mathrm{m}$ of $\mathrm{Al}$ and $1 \mu \mathrm{m}$ of Lexan. The Henke tables were used to calculate this transmission (51)

calculated for the wavelength range seen in the film by looking up the respective transmissions from tables of measured values taken by Henke (51). As the filters used here are composed of two sections (aluminum and Lexan), the total filter transmission goes as

$$
T_{\text {filter }}=T_{A l} \times T_{\text {Lexan }} .
$$

Thus the total transmission (figure 2.20) is the product of the transmission through the Aluminum and the transmission through the Lexan. The spectra is then divided by this filter transmission to ensure that we're only looking at the spectra from the foil and pinch. Then we convert from exposure units into intensity units by applying a known film response to the spectra.

Once all of the above is applied to both slit spectra, they can be averaged together. An important point of this is that due to the nature of the slits in certain shots (when the slits are different sizes) the amount of photons will be more or less, leading to an overall shift in intensity without affecting the spectra itself. In order to account for this the spectra with the higher value of intensity is treated as the standard, and the lower-intensity spectra is scaled to match this. This is done for several sections of the spectra, to ensure more accurate scalings 


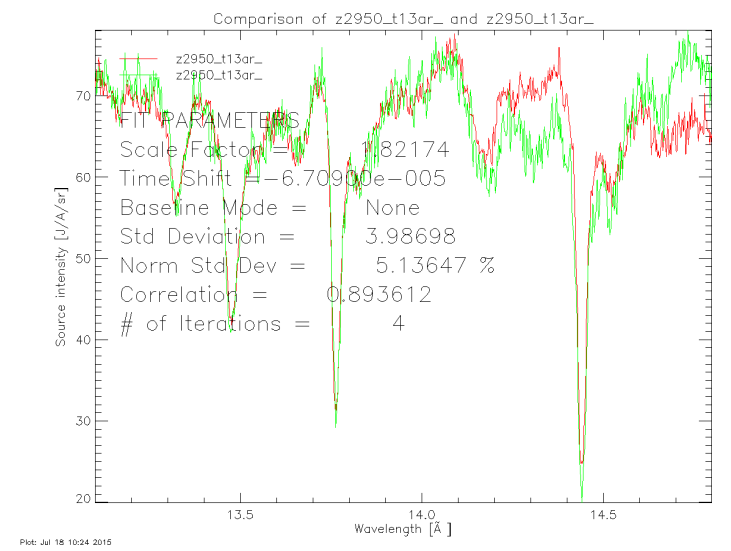

Figure 2.21: A subsection of spectra (one containing He-like F lines) that was shifted before averaging. The shift does not affect the spectral features, only the baseline. The bottom slit spectra (red) was scaled by a factor of 1.8 to align it with the top slit spectra (green)

(figure 2.21). These are then stitched back together to form a total scaled spectra, which is then averaged with the unscaled spectra. An important note is that this can lead to a rise in processing artifacts if the scaling factors on two sides of a split are not the same, so it is important that the locations for a spectral split are not near any spectral features. It is also important to point out that these experiments do not use absolute measurements, but relative measurements so this process does not disturb the results in any way.

The final step of the data processing is to go from transmission (in intensity units) to line transmission (in transmission units). In order to do this, the baseline of the spectra must be divided out of the total transmission. In order to do this, T. Nagayama has a program to load in spectra, and users can select spectral features to be removed from the calculations. Then a Butterworth filter is applied to the remaining spectra to smooth it and determine the baseline. This total transmission is then divided by the baseline, leaving only line transmission. Figure 2.22 shows the total transmission as well as the determined baseline, and Figure 2.23 shows how the distribution of points around the baseline is, which is generally Gaussian in nature. A typical $4 \mu \mathrm{m}$ tamped shot's KAP spectra can be seen in figure $2.24 \mathrm{a}$, while a typical $7 \mu \mathrm{m}$ tamped, axially resolved shot is present in figure 2.25, and $15 \mu \mathrm{m}$ tamped spectra is shown in figure 2.26. 


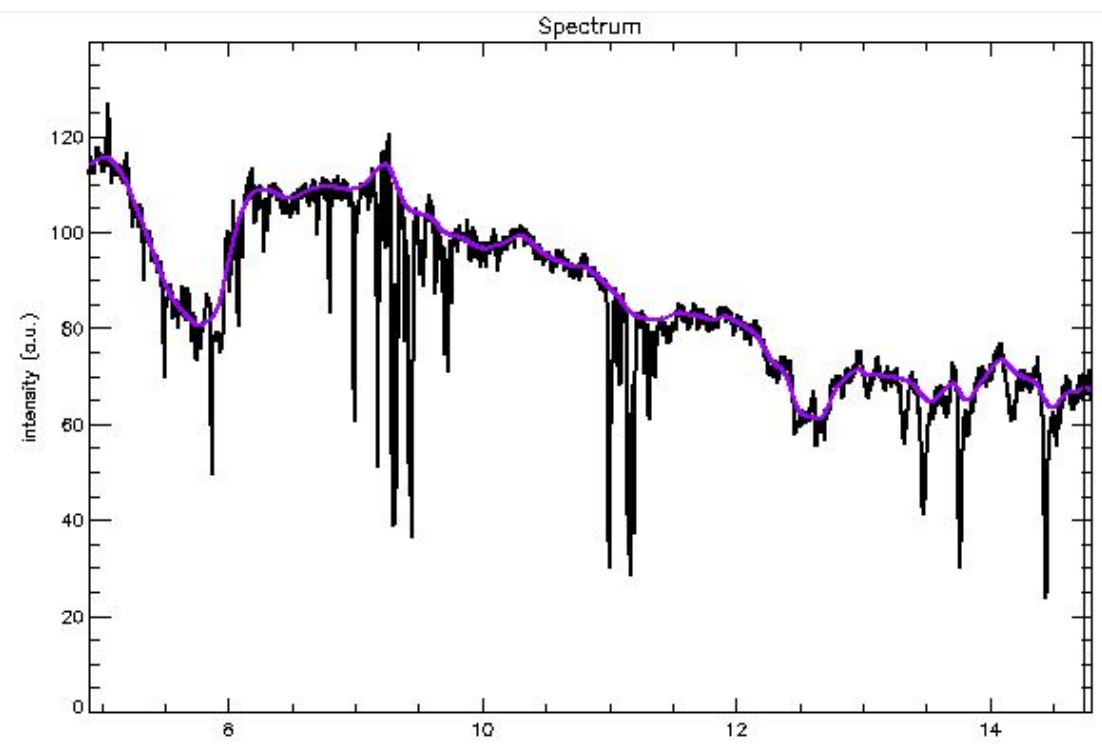

Figure 2.22: The background produced by removing spectral features and applying the Butterworth filter (purple) contrasted with the spectra (black).

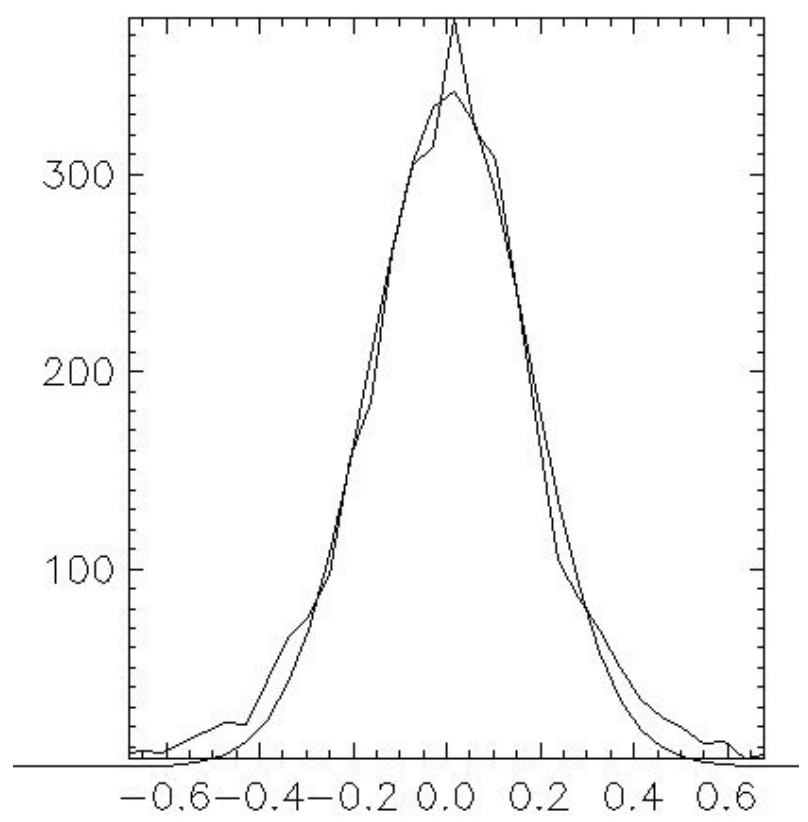

Figure 2.23: The deviations from the determined background are fitted with a Gaussian. In general, the deviations follow Poisson statistics, resulting in a good fit. 


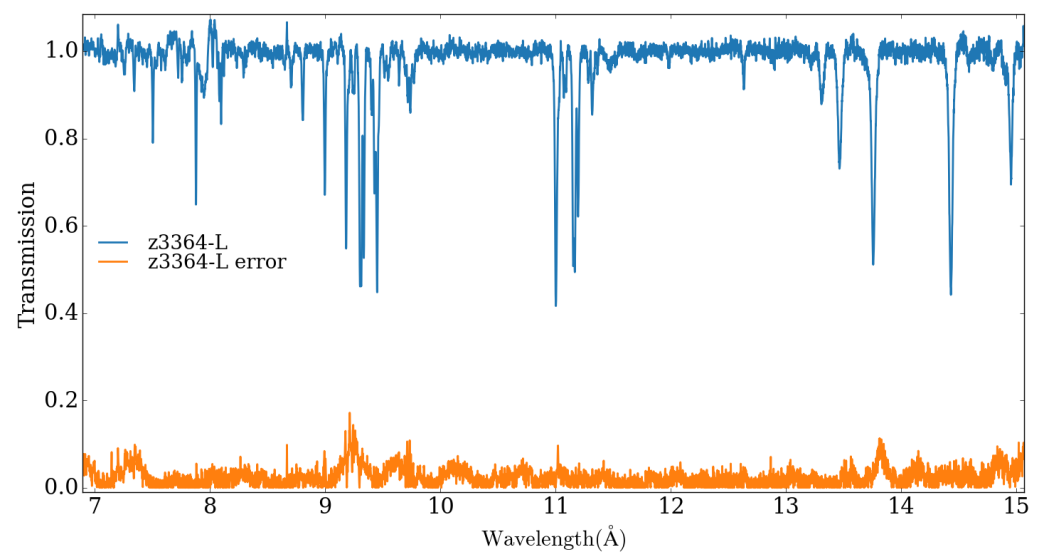

(a) Spectra gathered on z3364 using a KAP crystal in the TIXTL (blue) and the error (orange). The error is the standard deviation from averaging the two slits together. This is a very typical $4 \mu m$ tamped shot.

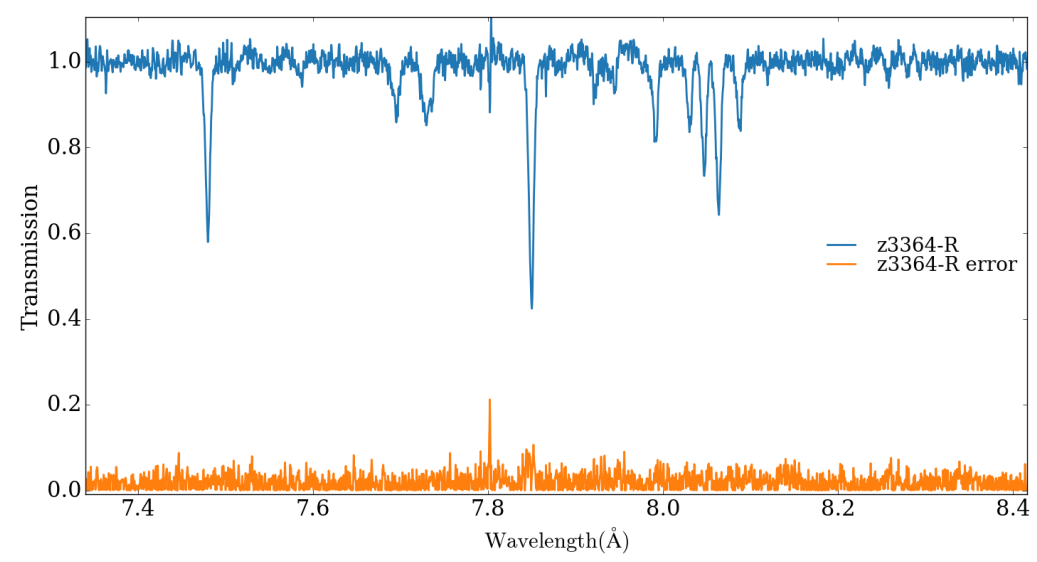

(b) Spectra gathered on z3364 using a TAP crystal in the TIXTL, along with the error.

Figure 2.24: Using both a KAP and a TAP on a $4 \mu m$ shot, a full spectrum can be seen and with enough resolution to detect line broadening. 


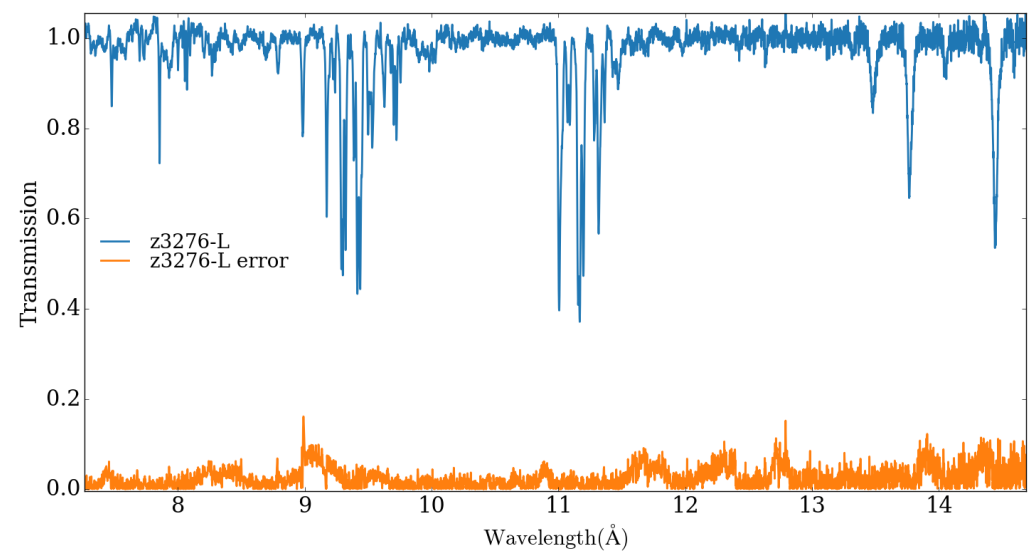

(a) Spectra gathered on z3276 using a KAP crystal in the TIXTL, along with the error.

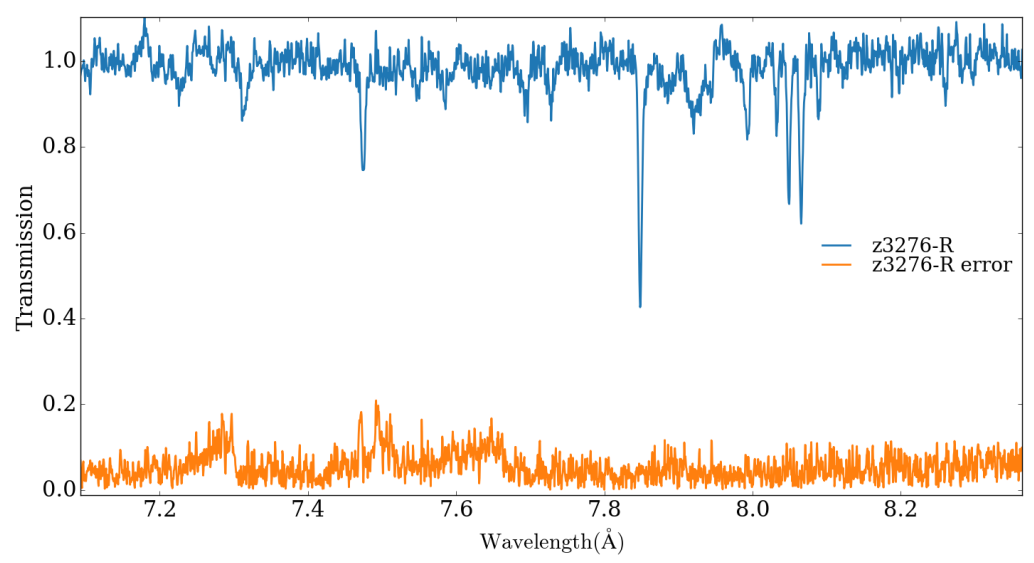

(b) Spectra gathered on z3276 using a TAP crystal in the TIXTL, along with the error.

Figure 2.25: Using both a KAP and a TAP on a $7 \mu m$ shot, a full spectrum can be seen and with enough resolution to detect line broadening. 


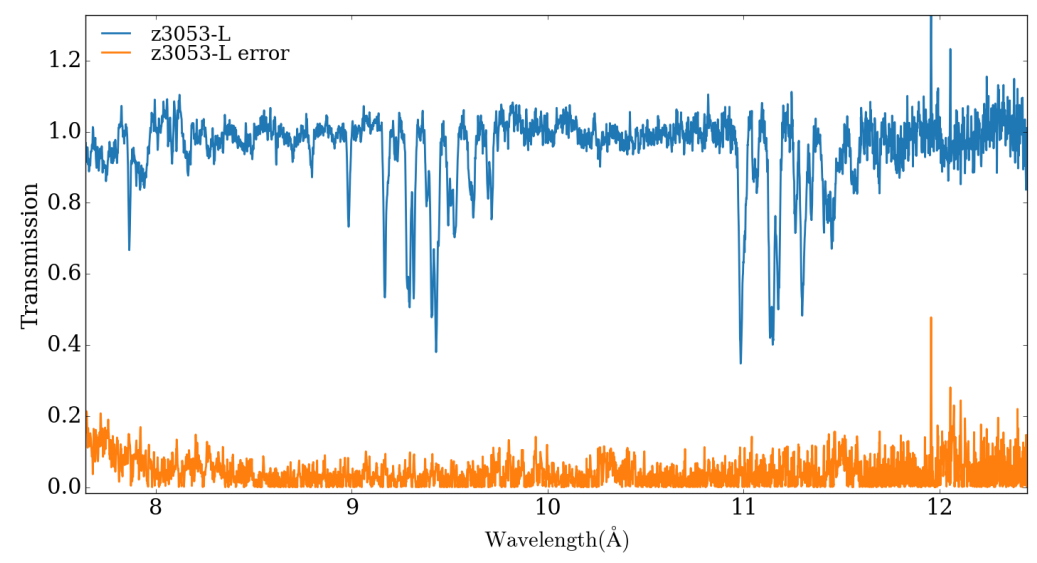

Figure 2.26: Spectra gathered on z3053 using a KAP crystal in the TIXTL (blue) and the error (orange). The $15 \mu \mathrm{m}$ tamped shots do not have any spectral features past $12 \AA$, most likely due to the attenuation from the large amount of $\mathrm{CH}$. There were no TAP shots with this tamper. 
2. EXPERIMENTS 


\section{3 \\ Determining the Usefulness of Isoelectronic Line Ratios as a Temperature Diagnostic}

Science is a beautiful gift to humanity; we should not distort it. -A.P.J. Abdul Kalam

\subsection{Previous Work}

Line ratios are used often to determine the temperature in a HED plasma (52), (50), (53), (54), (55). While line ratio techniques may not be as accurate as other methods (56), they are much easier to preform and can also work in absorption. Inter-stage line ratios are often used as a temperature diagnostic, such as in Bailey et al 's work on Fe Opacity in stellar interiors (3), where a tracer layer of $\mathrm{Mg}$ is used to infer temperature via line ratios comparing Lyman lines and He-like lines. In emission, inter-stage lines are often used to compare the results from isoelectronic line ratios (57), (58), to ensure that isoelectronic lines do indeed work. This is how we will use inter-stage line ratios, only in absorption.

Isoelectronic line ratios were originally proposed by Marjoribanks (59) in emission, who conducted experiments using the Omega Laser to ensure that isoelectronic line ratios could be used to determine temperature. Since, they have been 


\section{DETERMINING THE USEFULNESS OF ISOELECTRONIC LINE RATIOS AS A TEMPERATURE DIAGNOSTIC}

used in many instances to determine temperature from emission spectroscopy, including work being done at the National Ignition Facility (NIF) in experiments towards achieving fusion (1). This is largely due to isoelectronic line ratios consistency across time (60), (61), across different radiation drivers and across differing electron densities (62). However, isoelectronic line ratios have only been used in emission, not in absorption. It is our aim to show that isoelectronic line ratio techniques can be used to determine plasma temperatures in absorption spectroscopy.

\subsection{Theory}

The theory behind why line ratios predict temperature is truly quite simple. As a plasma heats up, the charge state distribution of the plasma will shift towards more highly ionized ions, such as going from a Be-like charge state with four electrons to a Li-like charge state with only three electrons; the ionization energy of an electron having been exceeded, allowing it to leave the ion and become part of the plasma. This can be seen in figure 3.1a; as the Na heats up, more of the ions tend toward the He-like charge state. The population of a charge state can be determined from the integral of a spectral feature in optical depth (12)

$$
n_{z}(q)=\frac{\kappa^{L}\left(\lambda_{q p}\right)}{\pi \lambda_{q p}^{2} r_{e} f_{q p}} .
$$

Here $\kappa^{L}\left(\lambda_{q p}\right)$ is the integral of the spectral feature in optical depth, $\lambda_{q p}$ is the wavelength of the transition, $r_{e}$ is the electron radius, $f_{q p}$ is the oscillator strength of the transition and $n_{z}(q)$ is the population of the initial state of the transition. With this, as long as the central wavelength and oscillator strength of a line is known, the only variable is the integral over the spectral feature. With an infinite resolving power and a full spectrum, the entire charge state distribution could be determined, and thus temperature. However, that type of measurement is exceedingly difficult. Two to three charge states can be calculated, and with those a ratio can be formed to determine temperature. For emission measurements, the initial state $q$ is actually the higher energy state before it decays and emits a photon, while for absorption the initial state is often the ground state. This 


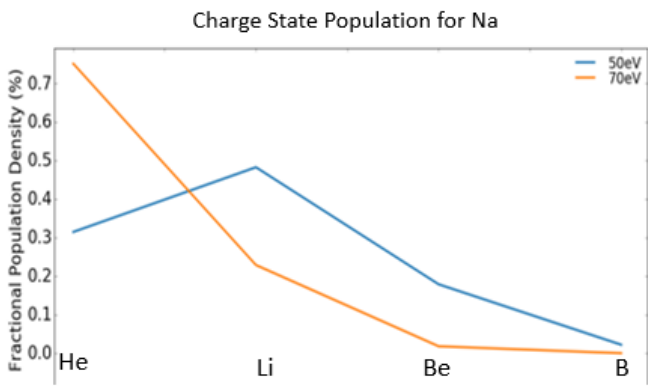

(a) The populations of charge states for $\mathrm{Na}$ at two different temperatures.

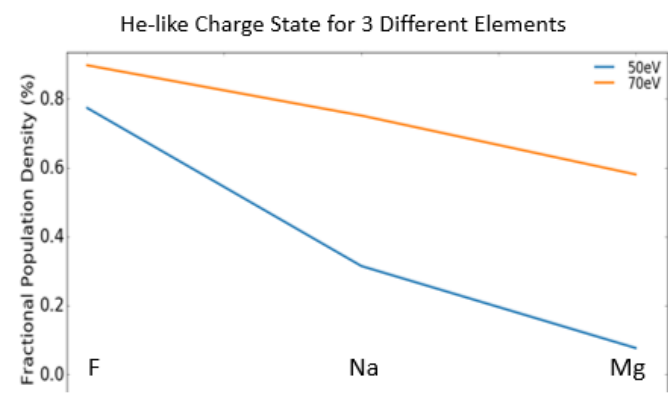

(b) He-like populations for $\mathrm{Na}, \mathrm{F}$ and $\mathrm{Mg}$ at two different temperatures.

Figure 3.1: Line ratio techniques for determining temperature are based off of the relative populations of charge states in an element. These graphs depict how these change between two different temperatures.

means that when taking a line ratio in emission, the two upper states of the transitions are compared, where as in absorption the ratio consists of the ground states of each transition.

The reason a ratio is needed is that if the charge state population of one state was taken, several temperatures could match up, but when two are taken, the number of possible temperature matches decreases. With a ratio, that number of temperature matches is generally one.

\subsubsection{Inter-Stage Line Ratios}

Inter-stage line ratios are often used in HED plasma as a temperature diagnostic in both absorption and emission (53), (3), (60), (54), (55). A main reason of this is due to the fact that a plasma will almost never be composed of only one charge state, allowing for spectral features of two adjacent charge states to be compared. Inter-stage line ratios also have the benefit that spectral lines from adjacent charge states can very easily be captured using just one spectrometer, as they appear very close in wavelength.

An inter-stage line ratio works by using the different ionization energies of adjacent charge states to measure the different fractional populations of those charge states. Ejecting an electron from a Li-like ion is much easier than ejecting 


\section{DETERMINING THE USEFULNESS OF ISOELECTRONIC LINE RATIOS AS A TEMPERATURE DIAGNOSTIC}

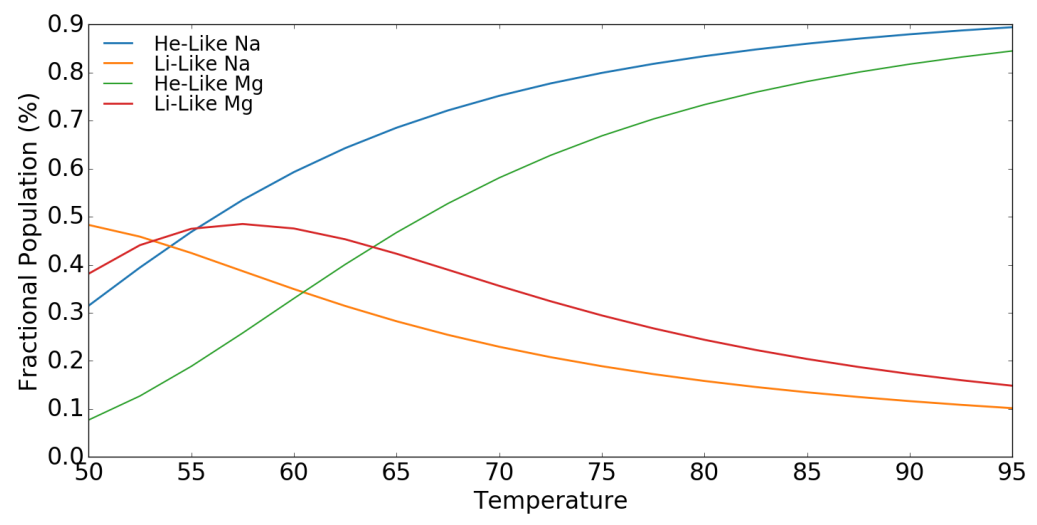

Figure 3.2: The He-like and Li-like charge state populations as functions of temperature. By taking ratios of these parameters temperature can be determined.

an electron from a He-like ion, and so before the energy is reached to achieve a He-like ion there will be a build-up of Li-like ions, leading to a higher fractional population (the reverse of this can be seen in figures 3.1a and 3.2). This can then be measured using spectral lines originating in the Li-like and He-like ions.

\subsubsection{Isoelectronic Line Ratios}

Isoelectronic line ratios are said to be more accurate than inter-stage line ratios because the lines compared in the ratio have identical origins, meaning any error in quantifying the population mechanisms would occur in both the numerator and the denominator, and thus cancel. Isoelectronic line ratios also have the benefit of being plentiful depending on the element mixture, and the relative line strengths can be manipulated based on stoichiometry.

How an isoelectronic ratio works is that it uses the different ionization energies for different elements to measure the different fractional populations of a charge state and compare it to the same charge state in a different element in the same plasma. A higher $\mathrm{Z}$ element will take more energy to ionize to the same charge state as a lower $\mathrm{Z}$ element, and thus the fractional population of a high charge state will be lower for a higher Z, and this difference can be taken advantage of. This can be seen in figures $3.1 \mathrm{~b}$ and 3.2 . 
The reason that isoelectronic line ratios have not been tested or used before in absorption likely lies in a simple fundamental difference between emission line ratios and absorption line ratios. That difference is that integrating line intensity gives the population of the initial state of the line, which in emission is the upper state before it decays back to the ground state. However, in absorption, this is the opposite; integrating optical depth gives the initial state which is the ground state. This can complicate interpretation if there is not a reliable spectral model. In absorption, there is also the problem of having an intense, broadband backlighter to provide for isoelectronic line ratios. Fortunately, that is not a problem with these experiments.

\subsection{Line fitting - Experiment}

The first step in computing a line ratio is converting the spectra from line transmission into optical depth. This is an easy transformation, as both quantities are unit-less and related by

$$
\begin{gathered}
T=e^{-\tau}, \\
\tau=-\ln (T),
\end{gathered}
$$

where $T$ is line transmission and $\tau$ is optical depth. It is important to note that this is not a linear conversion, and so optical depth of more than one (transmission of less than 0.35 ) can lead to some problems. Fortunately our spectra only has a few candidate lines that approach this.

It is imperative to convert our error in transmission into error in optical depth. Knowing the equation for propagation of errors, given by

$$
\sigma_{f}^{2}=\sum_{i}^{n}\left(\frac{\partial f}{\partial x_{i}}\right)^{2} \sigma_{x_{i}}^{2},
$$




\section{DETERMINING THE USEFULNESS OF ISOELECTRONIC LINE RATIOS AS A TEMPERATURE DIAGNOSTIC}

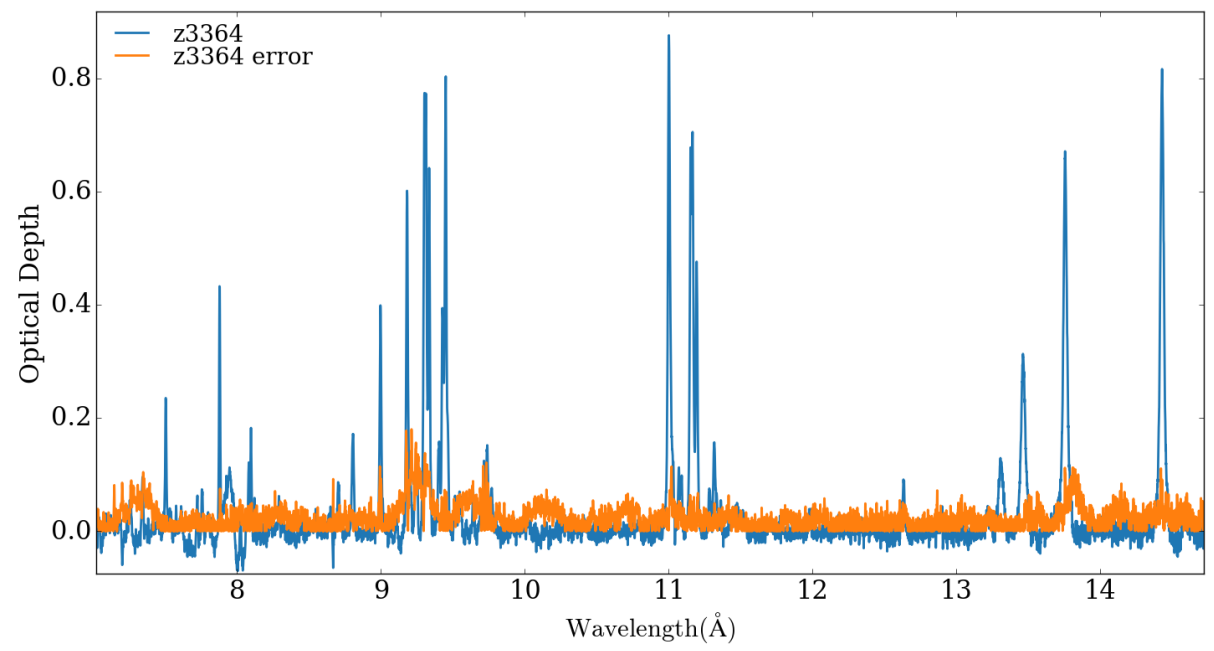

Figure 3.3: The KAP spectra from z3364 converted into optical depth (blue) and the associated error (orange).

we can substitute $f$ for equation $3.2 \mathrm{~b}$, and $\sigma_{x_{i}}$ for the error in transmission, $\sigma_{T}$. This gives us

$$
\sigma_{\tau}^{2}=\left(\frac{\partial(-\ln (T))}{\partial T}\right)^{2} \sigma_{T}^{2} .
$$

When evaluated this yields

$$
\begin{gathered}
\sigma_{\tau}^{2}=\left(-\frac{1}{T}\right)^{2} \sigma_{T}^{2}, \\
\sigma_{\tau}=\frac{\sigma_{T}}{T} .
\end{gathered}
$$

Using these, all spectra gathered from the shots on $\mathrm{Z}$ can be converted to optical depth along with their error (figure 3.3).

Once the spectra is in optical depth, we can apply our fitting routine to it. Our fitting routine fits three different profiles to a line in optical depth. A Lorentzian is given by

$$
L\left(x ; x_{o}, \gamma\right)=\frac{1}{\pi \gamma}\left[\frac{\gamma^{2}}{\left(x-x_{o}\right)^{2}-\gamma^{2}}\right]
$$


Where $\gamma$ is the half-width at half-maximum (HWHM) and $x_{o}$ is the centroid of the profile. A Gaussian is given as

$$
G\left(x ; x_{o}, \sigma\right)=\frac{1}{\sqrt{2 \pi \sigma^{2}}} e^{\frac{-\left(x-x_{o}\right)^{2}}{2 \sigma^{2}}},
$$

where $\sigma$ is the full-width at half-maximum (FWHM) for the profile. Finally we fit it with a Voigt profile

$$
V\left(x ; x_{o}, \gamma, \sigma\right)=\int_{-\infty}^{\infty} G\left(x^{\prime} ; x_{o}, \sigma\right) L\left(x^{\prime} ; x_{o}, \gamma\right) d x^{\prime} .
$$

As this is a convolution of the Gaussian and Lorentzian profiles it is much easier to fit a pseudo-Voigt, given by

$$
V(x ; \eta)=\eta L\left(x ; x_{o}, \gamma\right)+(1-\eta) G\left(x ; x_{o}, \sigma\right),
$$

where $\eta$ is a function of the Lorentzian FWHM $\left(f_{l}\right)$, Gaussian FWHM $\left(f_{g}\right)$ and total FWHM (f), which can be stated within one percent accuracy as (63)

$$
\begin{gathered}
\eta=1.366 \frac{f_{l}}{f}-0.477\left(\frac{f_{l}}{f}\right)^{2}+0.111\left(\frac{f_{l}}{f}\right)^{3} \\
f=\left(f_{g}^{5}+2.69 f_{g}^{4} f_{l}+2.428 f_{g}^{3} f_{l}^{2}+4.47 f_{g}^{2} f_{l}^{3}+0.078 f_{g} f_{l}^{4}+f_{l}^{5}\right)^{1 / 5} .
\end{gathered}
$$

In this sense, knowing $\eta$ means we know what percentage the profile is Lorentzian and what percentage it is Gaussian. This pseudo-Voigt, along with a Gaussian and Lorentzian and a static horizontal line for background, are used to calculate the "profile of best fit" for any particular spectral feature. This is done by using a $\chi^{2}$ method, with the lowest $\chi^{2}$ value taken as the best fit. Examples of this can be seen in figures 3.4, 3.5 and 3.6. The Voigt profile's integral (and width) were used, as the instrument function produces a Voigt profile, as does the combination of Doppler and Stark broadening.

An important note for the isoelectronic line ratio study is that all the line ratios discussed in this chapter come from KAP data. This is due to the spectrum from a KAP crystal having a much larger range, capturing spectral features from the three elements of interest, while the TAP often only captured lines from one 


\section{DETERMINING THE USEFULNESS OF ISOELECTRONIC LINE RATIOS AS A TEMPERATURE DIAGNOSTIC}

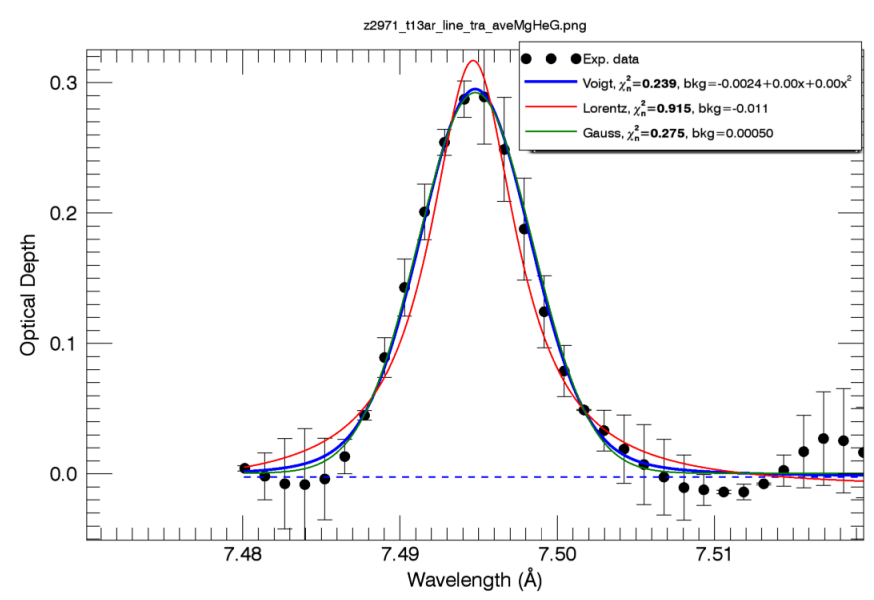

Figure 3.4: The fit of the $M g-H e \gamma$ line from shot z2971 in optical depth. The line is fit with a Gaussian (green), Lorentzian (red) and Voigt (blue). The Voigt is generally the best fit and is what is used to determine the integral and width of the line.

element $(\mathrm{Mg})$. The TAP fits could be used for this work, as it is just a different instrument function, and so should not affect the areas of lines, as a convolution with a Voigt profile does not affect the integral of whatever it was convolved with.

\subsection{PrismSPECT}

When computing temperatures from line ratios, a detailed atomic model is often employed, such as RATION (59) or FLY (60), to solve the complex rate equations for various temperatures and densities. In our work, the model used is PrismSPECT (64) (65).

PrismSPECT is a collisional-radiative model for producing spectra. It includes the various processes discussed in Chapter 1, such as electron-impact ionization, recombination, radiative recombination, photoionization and photoexcitation to name a few. PrismSPECT can be run in a non-LTE mode or with a population of hot electrons, but our experiments do not seem to indicate either of those being the case, so a Maxwellian distribution was assumed. The photoionization cross sections, oscillator strengths, autoionization rates and energy 


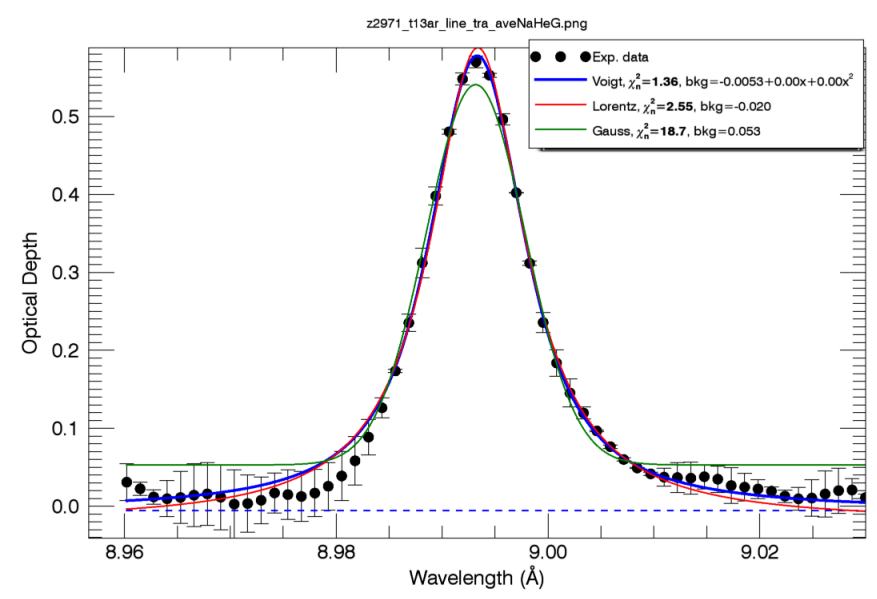

Figure 3.5: The fit of the $N a-H e \gamma$ line from shot z2971 in optical depth. It is more obvious in this fit that the Voigt is the best fit for the line. The area of this line will be compared to the area from the $M g-H e \gamma$ as an isoelectronic line ratio to determine temperature.

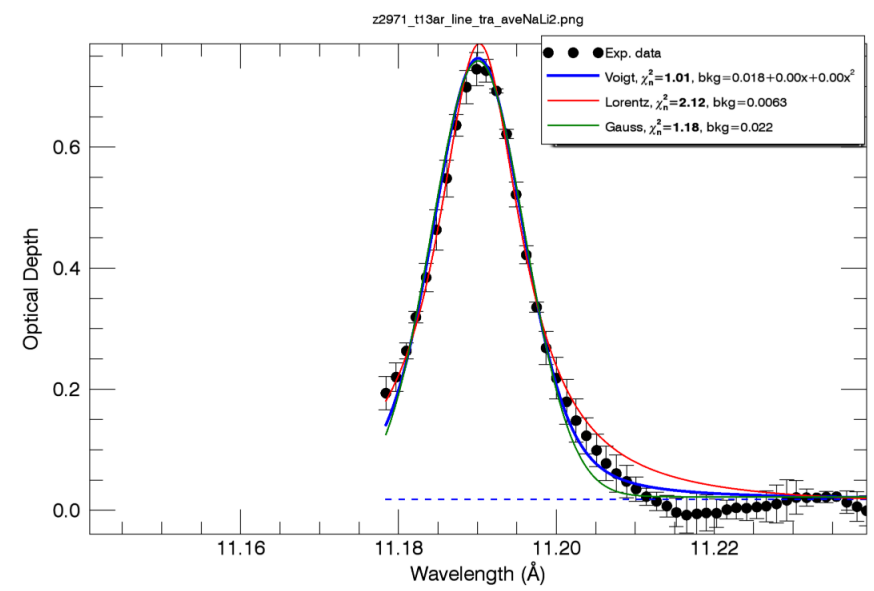

Figure 3.6: The fit of the $N a-L i$ line from shot z 2950 in optical depth. The fit is clipped on the low wavelength side due to the presence of another line. This is not too concerning, as the short wavelength side agrees with the fit very well. 


\section{DETERMINING THE USEFULNESS OF ISOELECTRONIC LINE RATIOS AS A TEMPERATURE DIAGNOSTIC}

levels are calculated using the ATBASE package (66), which uses a Hartree-Fock model, and fed into PrismSPECT for the four different elements. For the ATBASE files used for this work, the "all levels" model was used, meaning that all possible configurations for each charge state were used, there was no truncation to superconfigurations. This was due to not knowing what lower ionization levels may be present at which temperatures when the PrismSPECT database was created at the beginning of these experiments. This was also done to ensure the most accurate outcome and reduce possible user-error.

When setting up PrismSPECT, the relative abundances of the four elements, found in table 2.2 were used, as well as the thicknesses found there. As previously stated, an "all levels" model was used for all four elements. It is important to note that while spectra from only three elements is analyzed, all four elements must be present in the calculations. This is because the oxygen will provide more electrons, which will impact Stark broadening (to be examined next chapter), and also because of the possibility of ion collisions which while small, should be taken into account.

Within PrismSPECT, there are three variables to be entered; temperature, ion density and areal density, of which only two may be put in as a table. For these experiments, we assume a 1-D expansion, meaning that areal density is constant, thus temperature and ion density are input as tables, so one PrismSPECT run can yield multiple spectra for different combinations of temperature and density. As we assume LTE, the temperature listed here is the plasma temperature, not ion or electron temperature. Ion density is used as opposed to electron density, as the electron density $\left(n_{e}\right)$ will be calculated by determining the average charge state of the plasma $(\bar{Z})$ and multiplying the ion density $\left(n_{i}\right)$ shown by

$$
n_{e}=\bar{Z} n_{i}
$$

The temperatures used for the $4 \mu \mathrm{m}$ and $7 \mu \mathrm{m}$ tamped cases were $40-75 \mathrm{eV}$ with $1 \mathrm{eV}$ increments and ion densities of $2.25 \times 10^{20}-4 \times 10^{20}$ ions/cc with increments of $0.25 \times 10^{20}$ ions/cc. The temperature range was chosen based off a cursory overview of charge states as a function of temperature, and the density range from a back-of-the-envelope fit of FWHM for the F-Her line. The $15 \mu \mathrm{m}$ 
tamped case does not have any $\mathrm{F}$ lines, so the density range used is wider. The temperature range for the $15 \mu \mathrm{m}$ case is lower due to the suspected attenuation of x-rays from the increase in tamper. The ranges for this last case were 35$65 \mathrm{eV}$ with $1 \mathrm{eV}$ increments, and $2 \times 10^{20}-4.75 \times 10^{20}$ ions/cc with increments of $0.25 \times 10^{20}$ ions $/ \mathrm{cc}$.

PrismSPECT outputs absorption spectra assuming a perfect backlighter at all wavelengths. This then has our instrument function (figure 2.13) applied to it so that the resulting spectra is convolved using the same process as our data.

\subsubsection{Line fitting - PrismSPECT}

Much like the way we treat the experimental data, the first thing that is done with the PrismSPECT data is to convert it into optical depth. This is done using the same method that was applied to the data, so as to avoid possible problems with the conversion. Once the spectra is in optical depth, the lines are fit. For this data, only a Pseudo-Voigt is used to fit the lines, this is done largely because the Pseudo-Voigt is the most accurate fit for the spectral features due to both the nature of the instrument broadening and the broadening from within the plasma, but also to save time, as over 300 spectra must be fit for each line. Examples of these fits can be seen in figure 3.7 .

Once two lines are fit for all the combinations of temperature and density, the areas for each line can be combined in the form of a ratio. A point of note here is that we always have the lower wavelength line on the top of the ratio. There is no physical reason to do this, and should not impact the results as long as we are consistent.

The line ratio values are then plotted as functions of temperature, with the different densities as different lines within the plot. This allows for a visual representation of the line ratio's ability to predict temperatures at different densities. This can be seen in figure 3.8 . 


\section{DETERMINING THE USEFULNESS OF ISOELECTRONIC LINE RATIOS AS A TEMPERATURE DIAGNOSTIC}

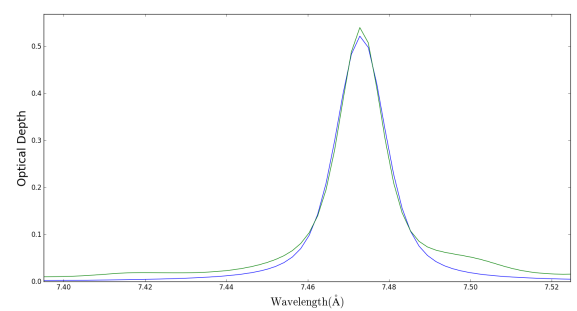

(a) The $M g-H e \gamma$ line from PrismSPECT (green) compared to the fit of the line (blue)

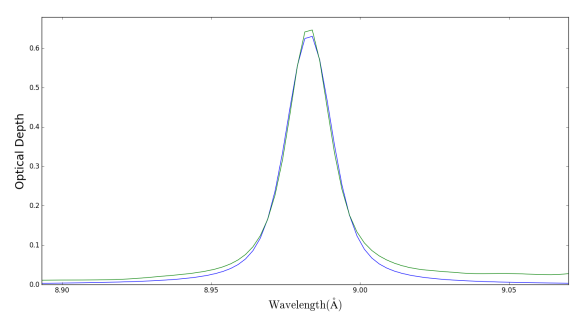

(b) The $\mathrm{Na}-\mathrm{He \gamma}$ line from PrismSPECT (green) compared to the fit of the line (blue)

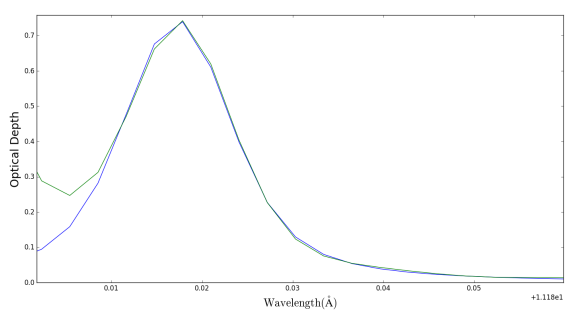

(c) A $N a-L i$ line from PrismSPECT

(green) compared to the fit of the line

(blue). It is important to note that the

lower wavelength section does not match

as well as the longer wavelength due to

the presence of another $N a-L i$ line.

Figure 3.7: The routine fits a designated line in PrismSPECT with a Voigt profile, then repeats this process for every combination of temperature and density provided. 


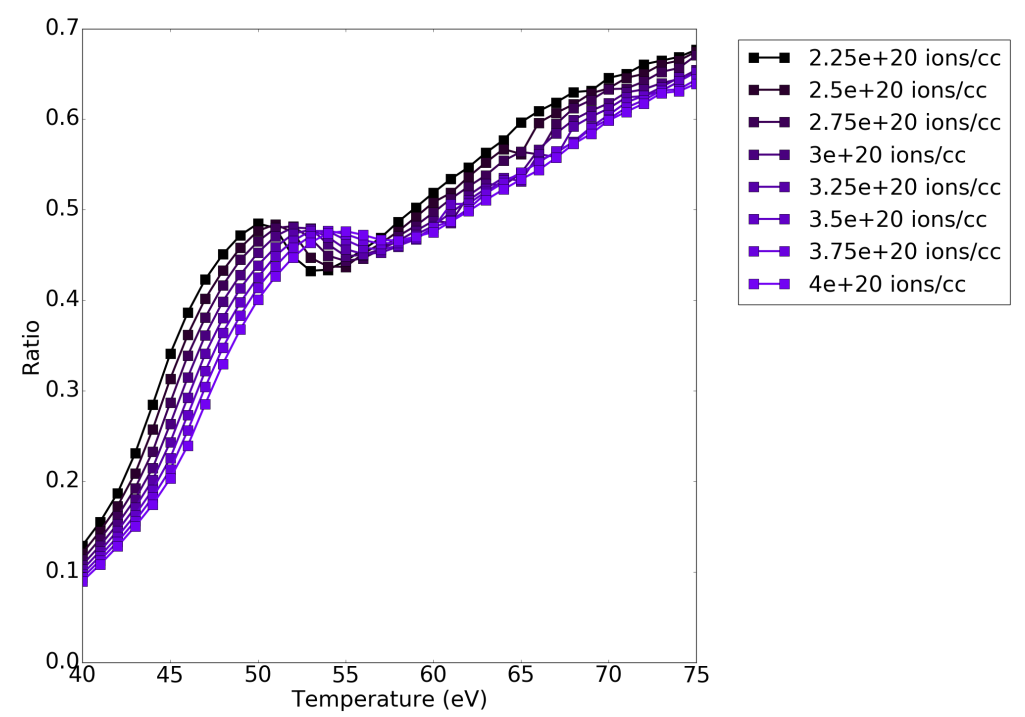

Figure 3.8: By plotting the $M g-H e \gamma$ integral over the $\mathrm{Na}-\mathrm{He \gamma}$ integral for all of the PrismSPECT runs we can see how the ratio value changes as a function of temperature for each different density.

\subsection{Analysis}

Once all the fitting has taken place, the ratios determined from experimental data need to be compared to the ratio values determined from PrismSPECT. This is easily done by overlaying the experimental values with the PrismSPECT generated values, as seen in figures 3.9 and 3.10. All the points that the experimental ratio and its error cross through are equally valid temperature values for that ratio, so they should all be taken into account. This is done by determining the lowest temperature point the experimental value intersects with and the highest temperature point the experimental value intersects with, then taking the average of those points and treating it as the temperature value, while the spread to the highest and lowest points is treated as the error. By treating it in such a way, all the points intersected can be considered part of the original value.

The temperatures determined are then compared across different shots of the same tamper thickness. As there are generally more than one ratio for each tamper thickness, the values must be averaged. For this, the inverse of the error 


\section{DETERMINING THE USEFULNESS OF ISOELECTRONIC LINE RATIOS AS A TEMPERATURE DIAGNOSTIC}

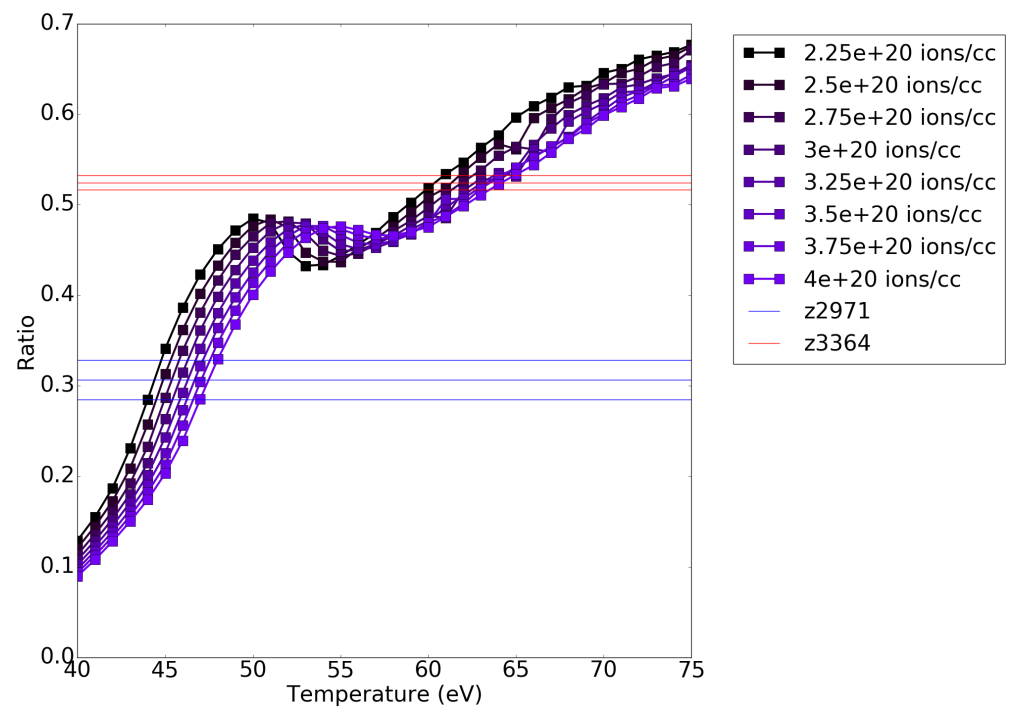

Figure 3.9: By overlaying the experimentally determined line ratios with the PrismSPECT line ratios, temperature can be determined. The data presented here is from z2971 (blue) and z3364 (red). A good fit for the $M g-H e \gamma$ line for z2950 could not be achieved so z2950 was left out.

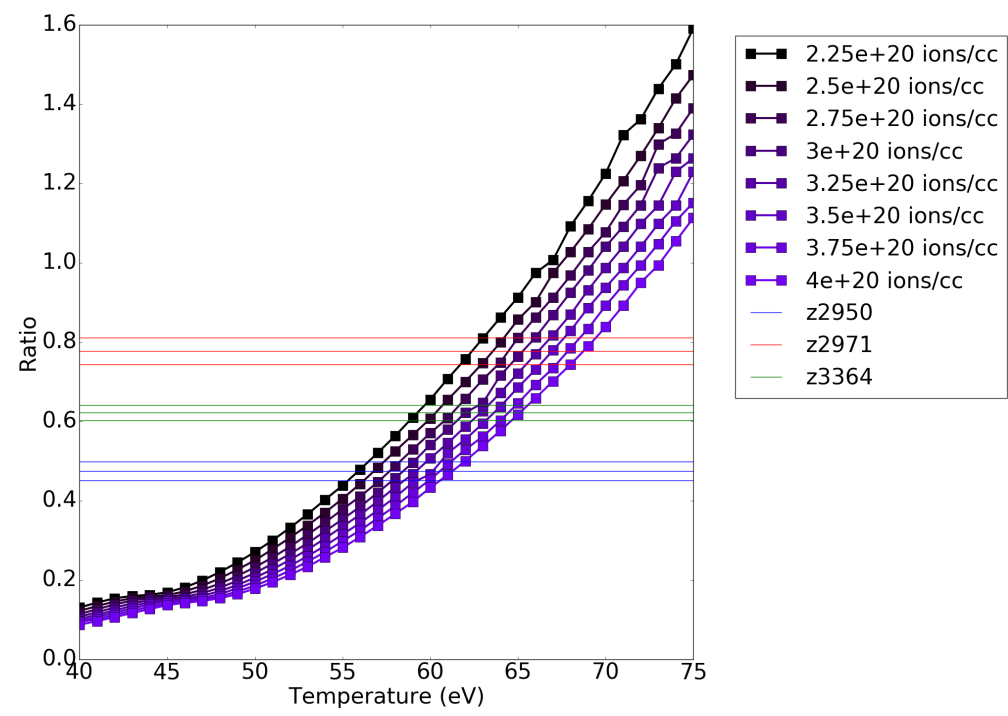

Figure 3.10: The inter-stage case for $N a-H e \gamma$ over one of the $N a-L i$ lines. The data presented here is from z2950 (blue), z2971 (red) and z3364 (green). 
is treated as a weighting factor, such that the average is given by

$$
<T_{\text {line }}>=\frac{\sum_{i=1}^{n} \frac{1}{\sigma_{i, \text { Line }}} T_{i, \text { Line }}}{\sum_{i=1}^{n} \frac{1}{\sigma_{i, \text { Line }}}},
$$

where $\mathrm{n}$ is the number of shots with the same configuration that yielded a result for this line ration. As these temperature values appear to be scatter across the experiments, the error for the average value is given by

$$
\left(\frac{\sigma_{\text {avg }}}{<T_{\text {Line }}>}\right)^{2}=\sum_{i=1}^{n}\left(\frac{\sigma_{i, \text { Line }}}{T_{i, \text { Line }}}\right)^{2} .
$$

\subsection{Results}

Once every line ratio yields one temperature, we have to compare the isoelectronic technique to the established inter-stage technique. this can be done by averaging all the isoelectronic line ratio temperatures and seeing if it falls within the standard deviation for the average inter-stage line ratio temperatures. The averaging process is the same as with individual shot data (using equation 3.12), but with a shift in how the overall standard deviation is calculated. As opposed to treating the points as scatter off of the average, we instead view them as a distribution from the average, and so the standard deviation is given by

$$
\sigma=\sqrt{\frac{\sum_{i=1}^{n} \sigma_{i}^{-1}\left(T_{i}-<T>\right)^{2}}{\frac{n-1}{n} \sum_{i=1}^{n} \sigma_{i}^{-1}}} .
$$

\subsubsection{4 micron tamped}

The individual shot results for the $4 \mu \mathrm{m}$ tamped case can be found in table 3.1. The $4 \mu \mathrm{m}$ tamped case has the most ratios, both isoelectronic and inter-stage, due in part to it reaching the highest temperature and having the least amount of $\mathrm{CH}$ to transmit through. This case is also nice in that all of the shots were axially resolving.

The average temperature found from isoelectronic line ratios is $56.9 \pm 3.2 \mathrm{eV}$, while the average temperature from inter-stage line ratios is $59.9 \pm 2.6 \mathrm{eV}$, this 


\section{DETERMINING THE USEFULNESS OF ISOELECTRONIC LINE RATIOS AS A TEMPERATURE DIAGNOSTIC}

\begin{tabular}{|c|c|c|c|c|}
\hline Line Ratio & $z 2950$ & $z 2971$ & $z 3364$ & Average \\
\hline $\begin{array}{l}\frac{M g-B e}{N a-B e} \\
\frac{M g-H e \alpha}{N a-H e \alpha} \\
\frac{M g-H e \beta}{F-H e \beta} \\
\frac{M g-H e \delta}{F-H e \delta} \\
\frac{M g-H e \delta}{N a-H e \delta} \\
\frac{M g-H e \gamma}{F-H e \gamma} \\
\frac{M g-H e \gamma}{N a-H e \gamma} \\
\frac{M g-L i}{N a-L i} \\
\frac{N a-H e \delta}{F-H e \delta} \\
\frac{N a-H e \epsilon}{F-H e \epsilon}\end{array}$ & $\begin{array}{l}48 \pm 2 \\
---- \\
---- \\
---- \\
---- \\
---- \\
---- \\
55.5 \pm 4.5 \\
61 \pm 3 \\
55.5 \pm 2\end{array}$ & $\begin{array}{l}58 \pm 5 \\
63 \pm 3 \\
56 \pm 3 \\
57.5 \pm 6.5 \\
57 \pm 9 \\
59 \pm 4 \\
46 \pm 2 \\
---- \\
59 \pm 3 \\
59 \pm 4\end{array}$ & $\begin{array}{l}49 \pm 5 \\
57 \pm 3 \\
61 \pm 3 \\
62 \pm 3 \\
63 \pm 4 \\
54.5 \pm 3.5 \\
62.5 \pm 2.5 \\
57 \pm 7 \\
59 \pm 4 \\
----\end{array}$ & $\begin{array}{l}50.44 \pm 5 \\
60 \pm 4.3 \\
58.5 \pm 4.5 \\
60.57 \pm 7.4 \\
61.2 \pm 10.4 \\
56.6 \pm 5.2 \\
53.3 \pm 3.1 \\
56.1 \pm 7.8 \\
59.7 \pm 4.1 \\
56.7 \pm 4.3\end{array}$ \\
\hline $\begin{array}{l}\frac{M g-H e \alpha}{M g-B e} \\
\frac{M g-H e \alpha}{M g-L i} \\
\frac{M g-H e \beta}{M g-B e} \\
\frac{M g-H e \beta}{M g-L i} \\
\frac{M g-H e \gamma}{M g-B e} \\
\frac{M g-H e \gamma}{M g-L i} \\
\frac{M g-L i}{M g-B e} \\
\frac{N a-H e \alpha}{N a-B e} \\
\frac{N a-H e \alpha}{N a-L i} \\
\frac{N a-H e \delta}{N a-B e} \\
\frac{N a-H e \delta}{N a-L i} \\
\frac{N a-H e \gamma}{N a-B e} \\
\frac{N a-H e \gamma}{N a-L i} \\
\frac{N a-L i}{N a-B e}\end{array}$ & $\begin{array}{l}60 \pm 2 \\
55.5 \pm 2.5 \\
60.5 \pm 2.5 \\
60.5 \pm 2.5 \\
---- \\
---- \\
61 \pm 3 \\
53.5 \pm 2.5 \\
51 \pm 2 \\
59 \pm 3 \\
62 \pm 4 \\
58 \pm 3 \\
60.5 \pm 2.5 \\
55.5 \pm 2.5\end{array}$ & $\begin{array}{l}60.5 \pm 2.5 \\
58 \pm 2 \\
60.5 \pm 2.5 \\
58 \pm 2 \\
61.5 \pm 3.5 \\
58.5 \pm 3.5 \\
66 \pm 4 \\
59.5 \pm 2.5 \\
55.5 \pm 2.5 \\
65 \pm 3 \\
66.5 \pm 3.5 \\
64 \pm 3 \\
65.5 \pm 3.5 \\
62.5 \pm 2.5\end{array}$ & $\begin{array}{l}61.5 \pm 4.5 \\
59.5 \pm 3.5 \\
63 \pm 4 \\
64 \pm 3 \\
63.5 \pm 4.5 \\
64.5 \pm 4.5 \\
61.5 \pm 7 \\
58.5 \pm 2.5 \\
59.5 \pm 2.5 \\
60 \pm 3 \\
62 \pm 4 \\
60 \pm 3 \\
62 \pm 3 \\
57.5 \pm 2.5\end{array}$ & $\begin{array}{l}60.5 \pm 4.1 \\
57.15 \pm 2.9 \\
61.1 \pm 3.7 \\
60.4 \pm 3 \\
62.4 \pm 5.7 \\
61.1 \pm 5.6 \\
62.8 \pm 5 \\
57.2 \pm 3 \\
55 \pm 2.8 \\
61.3 \pm 3.6 \\
63.6 \pm 4.7 \\
60.67 \pm 5 \\
62.4 \pm 3.7 \\
58.5 \pm 3.1\end{array}$ \\
\hline
\end{tabular}

Table 3.1: The temperatures determined from each line ration for each shot with $4 \mu m$ tamping, along with the averages. The ratios above the horizontal line are all isoelectronic, below are inter-stage. It should be noted that not all line ratios yielded a temperature for all shots. 


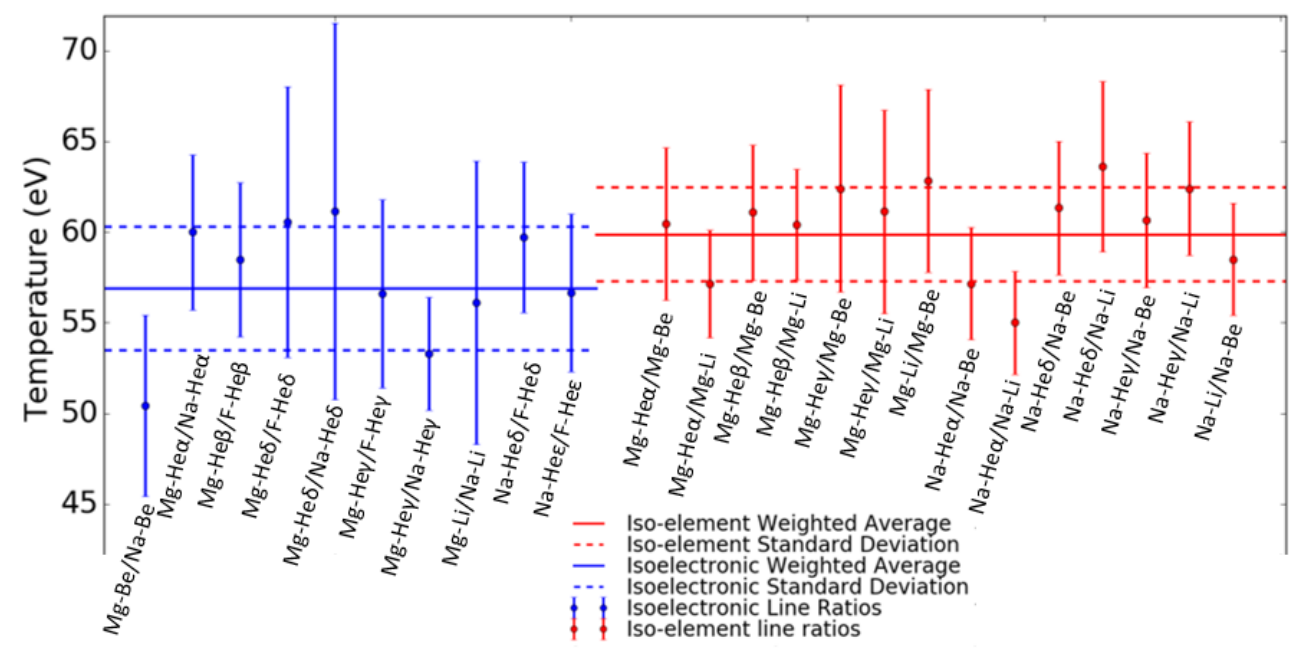

Figure 3.11: The average of the individual shot results for each ratio is plotted versus the temperature it predicts for the $4 \mu \mathrm{m}$ case. The average temperature for both the isoelectronic ratios and the inter-stage (iso-element) ratios is also shown. Overall there is agreement between the two.

can be seen in figure 3.11. We do see overlap between the iselectronic value and the inter-stage value, which lends itself to the belief that this technique does work and does agree with previous methods.

\subsubsection{7 micron tamped}

The $7 \mu m$ tamped case is special in that it is the only tamper thickness that spectra was gathered using both an axially resolving crystal and a radially resolving crystal. Because of this, the two different cases of spectra were analyzed separate to each other. The individual shot information for the axially-resolved case can be found in table 3.2, while the radially -resolved information is in table 3.3. It is important to note that the $7 \mu m$ tamped case has fewer isoelectronic line ratios than the $4 \mu m$ tamped case, but the same number of inter-stage line ratios. This is due to a loss of some of the high-n transitions in $\mathrm{Mg}$, most likely due to the slight decrease in temperature making the high-n lines blend with the noise. This problem is more pronounced in the radially-resolved $7 \mu \mathrm{m}$ tamped case, because in that case the high-n lines for $\mathrm{Na}$ are also under-resolved, thus the high-N lines 


\section{DETERMINING THE USEFULNESS OF ISOELECTRONIC LINE RATIOS AS A TEMPERATURE DIAGNOSTIC}

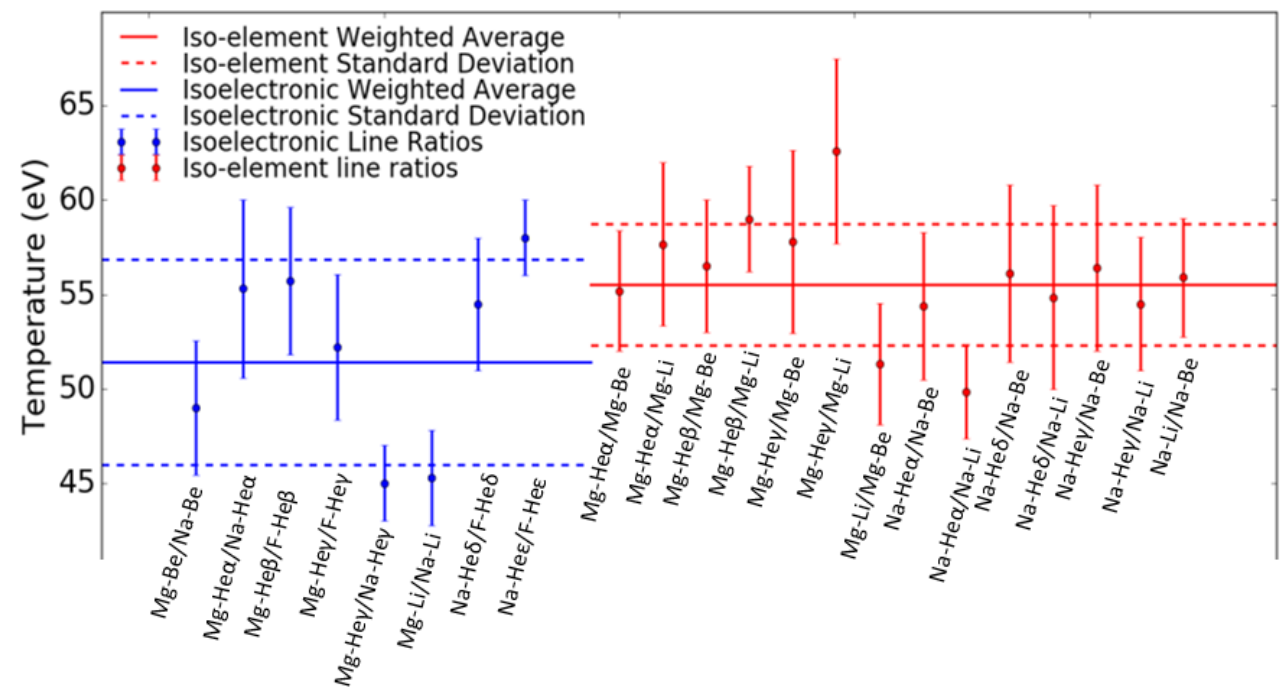

Figure 3.12: The average of the individual shot results for each ratio is plotted versus the temperature it predicts for the $7 \mu m$ axially resolving case. The average temperature for both the isoelectronic ratios and the inter-stage (iso-element) ratios is also shown, and there is agreement between the two. Both ratio techniques predict a lower temperature for the $7 \mu \mathrm{m}$ case than for the $4 \mu \mathrm{m}$ case by about $5 \mathrm{eV}$.

blending with the noise.

The average temperature found from isoelectronic line ratios for the axially resolved case is $51.4 \pm 5.4 \mathrm{eV}$, while the inter-stage line ratios have an average of $55.5 \pm 3.2 \mathrm{eV}$ (figure 3.12). The radially resolved case has the isoelectronic line ratios determine an average temperature of $50.4 \pm 6.1 \mathrm{eV}$ and the inter-stage line ratio average is $56.2 \pm 3 \mathrm{eV}$ (figure 3.13). The first thing to notice is that the axially resolved and radially resolved cases agree with each other very well. This can also be seen by looking at their individual shot values for temperature (tables 3.2 and 3.3). This should not come as a shock, as the only difference between the two cases is the instrument function, and convolving a function with any Voigt does not affect its area. This is also not a surprise as we have accounted for the different instrument functions. The second point of import is that we once again see agreement between the isoelectronic line ratios and the inter-stage line ratios, with the temperature values falling within the standard deviation of each other. 


\begin{tabular}{|c|c|c|c|}
\hline Line Ratio & $z 3286$ & $z 3276$ & Average \\
\hline $\begin{array}{l}\frac{M g-B e}{N a-B e} \\
M g-H e \alpha \\
N a-H e \alpha \\
M g-H e \beta \\
F-H e \beta \\
\frac{M g-H e \gamma}{F-H e \gamma} \\
\frac{M g-H e \gamma}{N a-H e \gamma} \\
M g-L i \\
N a-L i \\
\frac{N a-H e \delta}{F-H e \delta} \\
\frac{N a-H e \epsilon}{F-H e \epsilon}\end{array}$ & $\begin{array}{l}52.5 \pm 2.5 \\
55 \pm 4 \\
56 \pm 3 \\
46.5 \pm 2.5 \\
45 \pm 2 \\
45 \pm 2 \\
---- \\
58 \pm 2\end{array}$ & $\begin{array}{l}45.5 \pm 2.5 \\
55.5 \pm 2.5 \\
55.5 \pm 2.5 \\
59 \pm 3 . \\
---- \\
44.5 \pm 1.5 \\
54.5 \pm 3.5 \\
----\end{array}$ & $\begin{array}{l}49 \pm 3.6 \\
55.3 \pm 4.7 \\
55.7 \pm 3.9 \\
52.2 \pm 3.9 \\
45 \pm 2 \\
44.7 \pm 2.52 \\
54.5 \pm 3.5 \\
58 \pm 2\end{array}$ \\
\hline $\begin{array}{l}\frac{M g-H e \alpha}{M g-B e} \\
\frac{M g-H e \alpha}{M g-L i} \\
\frac{M g-H e \beta}{M g-B e} \\
\frac{M g-H e \beta}{M g-L i} \\
\frac{M g-H e \gamma}{M g-B e} \\
\frac{M g-H e \gamma}{M g-L i} \\
\frac{M g-L i}{M g-B e} \\
\frac{N a-H e \alpha}{N a-B e} \\
\frac{N a-H e \alpha}{N a-L i} \\
\frac{N a-H e \delta}{N a-B e} \\
\frac{N a-H e \delta}{N a-L i} \\
\frac{N a-H e \gamma}{N a-B e} \\
\frac{N a-H e \gamma}{N a-L i} \\
\frac{N a-L i}{N a-B e}\end{array}$ & $\begin{array}{l}55 \pm 2 \\
56.5 \pm 3.5 \\
56.5 \pm 2.5 \\
59 \pm 2 \\
55 \pm 4 \\
58 \pm 4 \\
52 \pm 2 \\
55.5 \pm 2.5 \\
49 \pm 2 \\
51.5 \pm 3.5 \\
51 \pm 3 \\
58.5 \pm 2.5 \\
56.5 \pm 2.5 \\
59.5 \pm 2.5\end{array}$ & $\begin{array}{l}55.5 \pm 2.5 \\
58.5 \pm 2.5 \\
56.5 \pm 2.5 \\
59 \pm 2 \\
59.5 \pm 2.5 \\
65.5 \pm 2.5 \\
50.5 \pm 2.5 \\
53 \pm 3 \\
50.5 \pm 1.5 \\
60 \pm 3 \\
60 \pm 4 \\
53.5 \pm 3.5 \\
52.5 \pm 2.5 \\
53 \pm 2\end{array}$ & $\begin{array}{l}55.2 \pm 3.2 \\
57.7 \pm 4.3 \\
56.5 \pm 3.5 \\
59 \pm 2.8 \\
57.8 \pm 4.9 \\
62.6 \pm 4.9 \\
51.3 \pm 3.2 \\
54.4 \pm 3.9 \\
49.86 \pm 2.5 \\
56.1 \pm 4.7 \\
54.9 \pm 4.9 \\
56.4 \pm 4.4 \\
54.5 \pm 3.5 \\
55.9 \pm 3.2\end{array}$ \\
\hline
\end{tabular}

Table 3.2: The temperatures determined from each line ratio for each shot with $7 \mu m$ tamping axially resolved, along with the averages. 


\section{DETERMINING THE USEFULNESS OF ISOELECTRONIC LINE RATIOS AS A TEMPERATURE DIAGNOSTIC}

\begin{tabular}{|c|c|c|c|}
\hline Line Ratio & $z 3286$ & $z 3276$ & Average \\
\hline $\begin{array}{l}\frac{M g-B e}{N a-B e} \\
\frac{M g-H e \alpha}{N a-H e \alpha} \\
\frac{M g-H e \beta}{F-H e \beta} \\
\frac{M g-H e \gamma}{F-H e \gamma} \\
\frac{M g-H e \gamma}{N a-H e \gamma} \\
\frac{M g-L i}{N a-L i}\end{array}$ & $\begin{array}{l}42.5 \pm 1.5 \\
55.5 \pm 1.5 \\
57 \pm 3 \\
---- \\
---- \\
48 \pm 5\end{array}$ & $\begin{array}{l}48 \pm 2 \\
58 \pm 2 \\
56 \pm 3 \\
56.5 \pm 2.5 \\
45.5 \pm 1.5 \\
45.5 \pm 1.5\end{array}$ & $\begin{array}{l}44.9 \pm 2.5 \\
56.6 \pm 2.5 \\
56.5 \pm 4.2 \\
56.5 \pm 2.5 \\
45.5 \pm 1.5 \\
46.1 \pm 5\end{array}$ \\
\hline $\begin{array}{l}\frac{M g-H e \alpha}{M g-B e} \\
\frac{M g-H e \alpha}{M g-L i} \\
\frac{M g-H e \beta}{M g-B e} \\
\frac{M g-H e \beta}{M g-L i} \\
\frac{M g-H e \gamma}{M g-B e} \\
\frac{M g-H e \gamma}{M g-L i} \\
\frac{M g-L i}{M g-B e} \\
\frac{N a-H e \alpha}{N a-B e} \\
\frac{N a-H e \alpha}{N a-L i} \\
\frac{N a-H e \delta}{N a-B e} \\
\frac{N a-H e \delta}{N a-L i} \\
\frac{N a-H e \gamma}{N a-B e} \\
\frac{N a-H e \gamma}{N a-L i} \\
\frac{N a-L i}{N a-B e}\end{array}$ & $\begin{array}{l}57 \pm 2 \\
55.5 \pm 2.5 \\
58 \pm 2 \\
59.5 \pm 2.5 \\
59.5 \pm 4.5 \\
57 \pm 7 \\
59 \pm 3 \\
51 \pm 3 \\
49.5 \pm 4.5 \\
57 \pm 4 \\
57 \pm 4 \\
52.5 \pm 3.5 \\
53 \pm 5 \\
51.5 \pm 5.5\end{array}$ & $\begin{array}{l}60 \pm 2 \\
58 \pm 2 \\
59 \pm 2 \\
61 \pm 2 \\
57.5 \pm 2.5 \\
60.5 \pm 2.5 \\
53.5 \pm 1.5 \\
53.5 \pm 2.5 \\
48.5 \pm 1.5 \\
53.5 \pm 2.5 \\
53.5 \pm 2.5 \\
56 \pm 3 \\
57.5 \pm 2.5 \\
57.5 \pm 2.5\end{array}$ & $\begin{array}{l}58.5 \pm 2.8 \\
56.9 \pm 3.2 \\
58.5 \pm 2.8 \\
60.3 \pm 3.2 \\
58.2 \pm 5.1 \\
59.6 \pm 7.7 \\
55.3 \pm 3.2 \\
52.4 \pm 3.9 \\
48.8 \pm 4.7 \\
54.5 \pm 5 \\
54.9 \pm 4.6 \\
54.4 \pm 4.7 \\
56 \pm 5.8 \\
55.6 \pm 6.4\end{array}$ \\
\hline
\end{tabular}

Table 3.3: The temperatures determined from each line ratio for each shot with $7 \mu m$ tamping radially resolved, along with the averages. The temperatures found here are within errors of the temperatures determined from the axially resolved $7 \mu m$ shots, with the difference being that higher-order transitions ( $\delta$ and $\epsilon$ lines) are not present. 


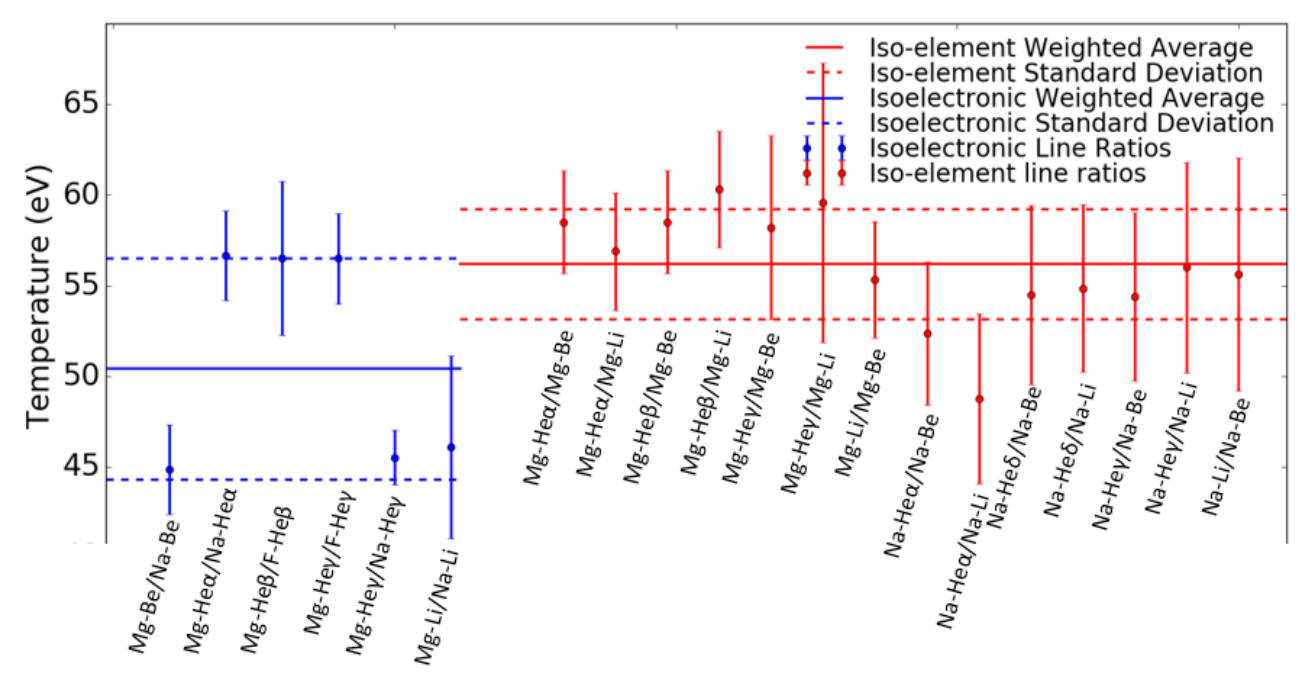

Figure 3.13: The average of the individual shot results for each ratio is plotted versus the temperature it predicts for the $7 \mu \mathrm{m}$ radially resolving case. These result and the axially resolving results agree, further showing that convolution does not affect the area of a line. This means that the two cases can be treated as a single setup when examining line ratios.

\subsubsection{5 micron tamped}

The $15 \mu \mathrm{m}$ tamped data was the most difficult to analyze, and thus has the fewest number of ratios, both isoelectronic and inter-stage. This is due to the same effects that harmed the $7 \mu \mathrm{m}$ shots (lower temperature, poor resolution, increase in tamper) but taken to an extreme. The $15 \mu \mathrm{m}$ shots do not have any $\mathrm{F}$ lines, meaning the amount in isoelectronic lines is greatly reduced. The effects of this reduce in the number of line ratios can be more easily seen in table 3.4.

The average temperature for the isoelectronic line ratios is $42.5 \pm 3.6 \mathrm{eV}$, while the inter-stage line ratios predict a temperature of $47.6 \pm 8$. The $15 \mu \mathrm{m}$ tamped case is the only case where the standard deviation for the isoelectronic line ratios is smaller than for the inter-stage line ratios. This is most likely due to the small number of isoelectronic ratios, all of which agree very well, which is seen in figure 3.14. Because of this shift in which line ratio method has the larger standard deviation, we can say with surety that the isoelectronic line ratio technique agrees with the inter-stage line ratio technique. 


\section{DETERMINING THE USEFULNESS OF ISOELECTRONIC LINE RATIOS AS A TEMPERATURE DIAGNOSTIC}

\begin{tabular}{l|l|l|l|l} 
Line Ratio & $\boldsymbol{z 2 9 5 0}$ & $\boldsymbol{z 2 9 7 1}$ & $\boldsymbol{z 3 3 6 4}$ & Average \\
\hline \begin{tabular}{l|l|l}
$M g-B e$ \\
$\frac{M g-B e}{N g-H e \alpha}$
\end{tabular} & $39.5 \pm 1.5$ & $41 \pm 2$ & $37.4 \pm 2.5$ & $39.5 \pm 3.6$ \\
$\frac{M a-H e \alpha}{N a-L i}$ & $50 \pm 4$ & $50 \pm 7$ & $41.5 \pm 1.5$ & $44.65 \pm 7.4$ \\
\hline & $44.5 \pm 2.5$ & $49.5 \pm 5.5$ & $45.5 \pm 2.5$ & $45.8 \pm 6.3$ \\
$\frac{M g-H e \alpha}{M g-B e}$ & $60.5 \pm 2.5$ & $57.5 \pm 2.5$ & $62.5 \pm 2.5$ & $60.12 \pm 4.4$ \\
$\frac{M g-H e \alpha}{M g-L i}$ & ---- & $40 \pm 3$ & ---- & ---- \\
$\frac{M g-H e \beta}{M g-B e}$ & $36 \pm 2$ & $38.5 \pm 1.5$ & $36 \pm 3$ & $37.1 \pm 4$ \\
$\frac{M g-H e \beta}{M g-L i}$ & $45.5 \pm 2.5$ & $50.5 \pm 5.5$ & $45 \pm 2$ & $46.1 \pm 6$ \\
$\frac{M g-L i}{M g-B e}$ & $57 \pm 2$ & $58 \pm 6$ & $60 \pm 2$ & $58.2 \pm 6.8$ \\
$\frac{N a-H e \alpha}{N a-B e}$ & $50.5 \pm 3.5$ & $50.5 \pm 2.5$ & $51.5 \pm 2.5$ & $50.9 \pm 5$ \\
$\frac{N a-H e \alpha}{N a-L i}$ & $56.5 \pm 3.5$ & $56 \pm 3$ & $55.5 \pm 2.5$ & $55.9 \pm 5.2$ \\
$\frac{N a-H e \gamma}{N a-B e}$ & $47 \pm 5$ & $51.5 \pm 2.5$ & $45.5 \pm 3.5$ & $48.5 \pm 6.8$ \\
$\frac{N a-H e \gamma}{N a-L i}$ & $39 \pm 2$ & $39.5 \pm 2.5$ & $51.5 \pm 4.5$ & $41.6 \pm 5$ \\
$\frac{N a-L i}{N a-B e}$ & $44 \pm 3$ & $47 \pm 2$ & $49 \pm 2$ & $47 \pm 4.2$
\end{tabular}

Table 3.4: The temperatures determined from each line ration for each shot with $15 \mu \mathrm{m}$ tamping, along with the averages. The ratios above the horizontal line are all isoelectronic, below are inter-stage. The temperatures from this tamper are generally lower than from the other tamper cases. 


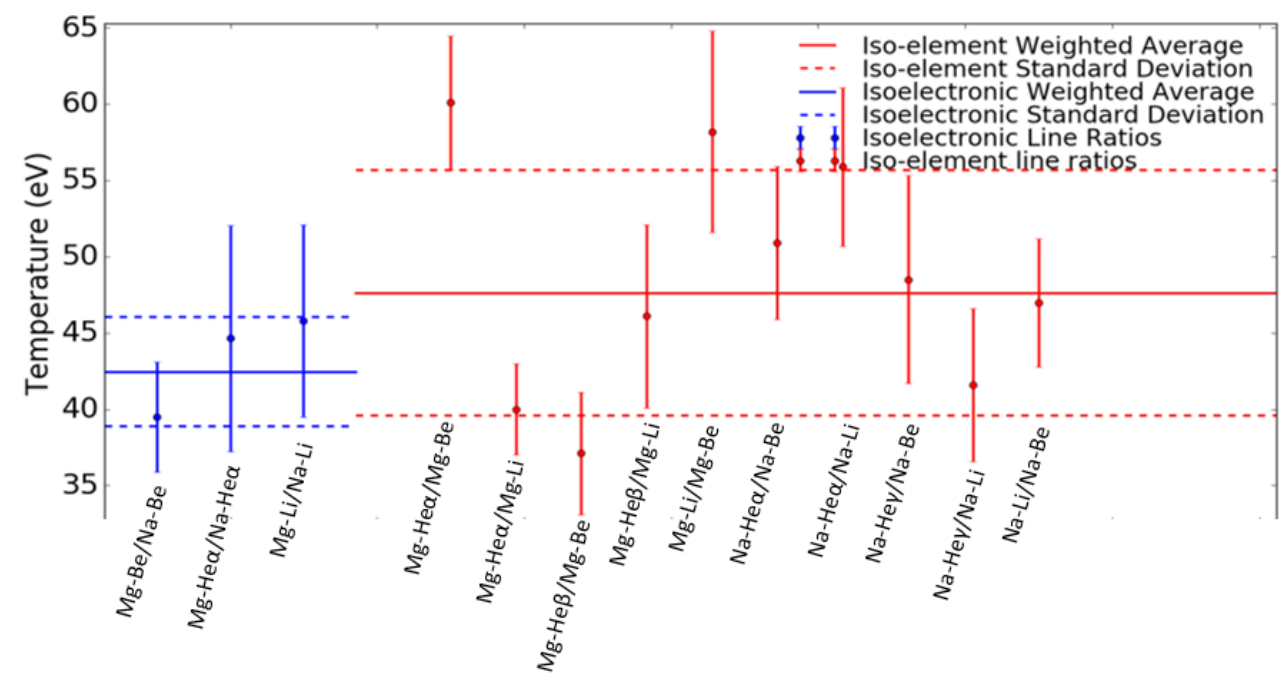

Figure 3.14: The average of the individual shot results for each ratio is plotted versus the temperature it predicts for the $15 \mu \mathrm{m}$ case. The average temperature for both the isoelectronic ratios and the inter-stage (iso-element) ratios is also shown, and there is agreement between the two. Both techniques have the $15 \mu \mathrm{m}$ case as the coldest, with a temperature $10-15 \mathrm{eV}$ lower than the $4 \mu \mathrm{m}$ case.

\subsubsection{Overall results}

Across all tamper thicknesses, the isoelectronic line ratios predicted a lower temperature than the inter-stage line ratios. A potential cause of this may be the isoelectronic line ratios indifference to the background radiation, or their sensitivity to time evolution. This may also be due to differences in the ionization energies for the elements as opposed to inter-element ionization energies. This would be an excellent point of study for future experiments, with possibly different elements used, or these same elements, but involving the oxygen lines as well. This could be done by using a Rubidium Acid Phthalate (RAP) crystal to capture longer wavelengths. The potential effects of spatial and temporal gradients, as well as next steps will be discussed more in the final chapter.

This work could be applied to any experiment that uses absorption spectroscopy, but it is more directed at absorption experiments that cannot use interstage line ratios. This may be due to a limited wavelength range in backlighter or spectrometer range. The isoelectronic line ratio technique can be tailored 


\section{DETERMINING THE USEFULNESS OF ISOELECTRONIC LINE RATIOS AS A TEMPERATURE DIAGNOSTIC}

to an experiments needs, all that is required is choosing the correct elements. Isoelectronic line ratios can also be employed in astronomy, such as determining temperature when investigating transmission through a supernova remnant. This application is tricky however, as it still requires detailed knowledge of the stoichiometry of the elements present within the nebula, which is not always known. 


\section{4 \\ Stark Effect on Multiple, Multiply-Ionized Elements}

Science may be described as the art of systematic over-simplification.

- Karl Popper

\subsection{Theory}

Line broadening occurs because of several factors, including the Heisenberg uncertainty principle, the thermal motion of emitters, the effects of electric fields near an emitter and due to the resolution of the instrument that is measuring the spectra-all of which we take into account. As with the preceding chapter, any spectrum produced by models is convolved with our instrument function (figures 2.13 and 2.14) so that the simulated spectra and our experimental spectra are as close to each other as possible.

Recall that broadening due to the Heisenberg uncertainty principle (1.5) is the natural line broadening. A typical value for this broadening is $\frac{\lambda}{\Delta \lambda}=1.5 \times 10^{6}$, or $\Delta \lambda \approx 9.2 \times 10^{-6} \AA$, which is insignificant compared to all other broadening mechanisms operating on the spectrum.

Doppler broadening is caused by the thermal motion of the emitters due to the changing wavelengths based on how fast and in what direction something is moving along the line of sight. Equation 1.9 estimates the full width at half 


\section{STARK EFFECT ON MULTIPLE, MULTIPLY-IONIZED ELEMENTS}

maximum (FWHM) due to Doppler broadening based on the emitter mass and temperature, assuming a Maxwellian distribution. If we assume a slightly higher temperature than what is seen in our experiments $\left(T_{i}=70 \mathrm{eV}\right)$, we find $\frac{\lambda}{\Delta \lambda}=6700$ for a Fluorine ion. For the $F-H e \gamma, \Delta \lambda=0.002 \AA$. When evaluating various models, the widths will differ by small amounts due to changes in the Doppler width.

Stark broadening, the accumulation of Stark shifts, was introduced in Chapter 1. The next two subsections explain in more detail the specific Stark broadening models used by the codes PrismSPECT and ATOMIC.

\subsubsection{PrismSPECT}

PrismSPECT uses semi-empirical formulas (67) to calculate Stark broadening in Lyman lines, with an applied Z-scaling to match the $\mathrm{H}$ lines to Lyman lines from other elements (68).For Stark broadening affecting He-like ions, PrismSPECT uses lineshapes calculated by D. Haynes or R. Mancini using the MERL code (69). MERL uses the static ion approach pioneered by Griem to determine Stark Broadening (70)

$$
I(\omega)=\int_{0}^{\infty} P(\epsilon)<J(\omega, \epsilon)>d \epsilon .
$$

Here, $P(\epsilon)$ is the probability of finding an electric microfield $\epsilon$ at the location of the emitter $(71)$, and $J(\omega, \epsilon)$ is the electron broadened profile for a given microfield strength at the same location, and the brackets denote a thermal averaging of the emitters. In MERL calculations, the microfield strength is based on Hooper's model (72)

$$
P(\epsilon)=\frac{1}{2 \pi} \epsilon \int_{0}^{\infty} T(l) \sin (\epsilon l) l d l,
$$

where,

$$
T(l)=\frac{1}{Q} \int \ldots \int e^{-\beta V+i \sum_{j} l\left(\frac{e r_{j}}{r_{j}^{3}}\left(1+\frac{r_{j}}{\lambda D}\right) e^{\frac{r_{j}}{\lambda D}}\right)} \Pi_{j}^{N} d r_{j}
$$


and

$$
V=\sum_{0=i<j}^{N} \frac{e^{2}}{r_{i j}} \exp \left[-\frac{r_{i j}}{\lambda_{D}}\right]
$$

and $\lambda_{D}$ is the Debye length given by

$$
\lambda_{D}=\sqrt{\frac{T_{e}}{4 \pi n_{e} e^{2}}} .
$$

Here, $r_{j}$ is the distance of the jth electron to the position at which the microfield strength is being calculated, $N$ is the number of electrons in the system, $\beta$ is defined as $1 / T_{e}, T_{e}$ is the electron temperature, and $Q$ is the partition function of the system (72). One property of these functions is the microfields become stronger as $n_{e}$ increases. This makes sense, as the more electrons present to contribute to the microfield, the stronger the microfield will be.

The other half of the Stark broadened lineshape depends on $J(\omega, \epsilon)$, which was formulated by Woltz (73) as

$$
J(\omega, \epsilon)=-\frac{1}{\pi} \operatorname{Im}\left[\sum_{i, f, i^{\prime}, f^{\prime}} \rho_{i} d_{f^{\prime}, i^{\prime}}\left[\omega-L_{R}(\epsilon)-M(\omega)\right]_{i^{\prime} f^{\prime}, i f}^{-1} d_{i, f}\right],
$$

where $i$ and $f$ represent the initial and final radiator states, $d$ is the radiator dipole operator and $\rho$ is the radiator density operator. $L_{R}(\epsilon)$ is the radiator Liouville operator which commutes with the Hamiltonian, shown by

$$
L_{R}(\epsilon) \equiv\left[H_{R}(\epsilon),\right]
$$

which is also written as

$$
L_{R}(\epsilon) \equiv\left(\frac{\partial H_{R}}{\partial r} \frac{\partial}{\partial p}-\frac{\partial H_{R}}{\partial p} \frac{\partial}{\partial r}\right)
$$

In equation 4.6, $M(\omega)_{i f, i^{\prime} f^{\prime}}$ is the electron broadening operator used in MERL. 


\section{STARK EFFECT ON MULTIPLE, MULTIPLY-IONIZED} ELEMENTS

This operator takes the form of

$$
\begin{array}{r}
M(\omega)_{i f, i^{\prime} f^{\prime}}=-\frac{2 i e^{4} n_{e}}{3 \hbar^{2}}\left(\frac{8 \pi m}{T_{e}}\right)\left[\delta_{f f^{\prime}} \sum_{i^{\prime \prime}} R_{i i^{\prime \prime}} \cdot R_{i^{\prime \prime} i^{\prime}} G\left(\Delta \omega_{i^{\prime \prime} f}\right)+\right. \\
\left.\delta_{i i^{\prime}} \sum_{f^{\prime \prime}} R_{f^{\prime} f^{\prime \prime}} \cdot R_{f^{\prime \prime} f} G\left(-\Delta \omega_{i f^{\prime \prime}}\right)-R_{i i^{\prime}} \cdot R_{f^{\prime} f}\left(G\left(\Delta \omega_{i f^{\prime}}\right)+G\left(-\Delta \omega_{i^{\prime} f}\right)\right)\right] .
\end{array}
$$

Here, $R$ is the radiator electron position vector and $G(\omega)$ is a function representing perturbing effects of surrounding electrons, calculated by taking the trace of the electron wave functions (using a Coulomb wave approximation) (74). It should be noted that the sums in equation 4.6 represent the trace of the product of two matrices, $d \times d$ and $\left[\omega-L_{r}(\epsilon)-M(\omega)\right]^{-1}$ where the rows and columns are indexed by $i$ and $f$, respectively. Another important point is that $M(\omega)$ increases linearly as $n_{e}$ increases.

\subsubsection{ATOMIC}

Another Theoretical Opacity Modeling Integrated Code, or ATOMIC, is part of the Los Alamos Suite of Relativistic (LASER) atomic physics codes (75). In the framework of LASER, separate codes, such as CATS (76), ACE (77) and GIPPER (78) solve the CR equations from Chapter 1 (excitation, de-excitation, electron impact excitation, photoionization, etc) using a distorted wave approximation to calculate the rate coefficients. These rate coefficients are then fed into ATOMIC (79), which can be run in LTE or nLTE approximations to solve for the populations of the atomic levels and create synthetic spectra.

Within ATOMIC, atomic structure calculations are performed by CATS (76), and Stark broadening is calculated using formalism of Lee (80). As with Stark broadening calculations within MERL, the Lee model uses equation 4.1 to formulate its broadening, however the Lee model uses different formalisms for both $P(\epsilon)$ and $J(\omega, \epsilon)$. For the electric microfields, the Lee model, and thus ATOMIC, uses the Adjustable-Parameter Exponent (APEX) model (81) (82). APEX gives the electric microfield probability as

$$
P(\epsilon)=\int \frac{d \lambda}{(2 \pi)^{3}} e^{-i \lambda \epsilon} F(\lambda)
$$


where, $F(\lambda)$ is the generating function,

$$
\begin{gathered}
F(\lambda) \equiv e^{G(\phi)} \\
G(\phi)=\int d r_{1} h_{1}\left(r_{1} \mid 0\right) R\left(r_{1}\right)\left(e^{i \lambda \cdot \frac{E(1)}{R\left(r_{1}\right)}}-1\right) .
\end{gathered}
$$

Here $h_{1}\left(r_{1} \mid 0\right)$ is the first Ursell cluster function, $E(i)$ is the electric field generated by the $i^{\text {th }}$ particle and $R(r)$ is the shielding parameter at location $\mathrm{r}(82)$.

The electron broadened profile takes the form

$$
J(\omega, \epsilon)=\left[\Delta \omega+(e \hbar) \epsilon \cdot d+\frac{i}{3 \hbar^{2}}(d \cdot d) G(\Delta \omega)\right]^{-1},
$$

where, the $\mathrm{G}$ function is the width operator,

$$
G(\Delta \omega)=n_{e} e^{2} \sqrt{\frac{32 m_{e} \pi}{T_{e}}} \int \frac{d k}{k} \frac{e^{-\frac{\omega^{2}}{k v_{T}}}}{\epsilon_{R}^{2}+\epsilon_{I}^{2}},
$$

which is integrated in Fourier space over the wavenumber $k$ and $v_{T}$ is the thermal velocity of the electrons. The quantities $\epsilon_{R}$ and $\epsilon_{I}$ are the real and imaginary parts of the dielectric function (80), given by

$$
\begin{gathered}
\epsilon_{R}=1+\frac{k_{D}^{2}}{k^{2}}\left(1-2 \frac{\omega}{k} \sqrt{\frac{m_{e}}{2 T_{e}}} \phi\left(\frac{\omega}{k} \sqrt{\frac{m_{e}}{2 T_{e}}}\right)\right), \\
\epsilon_{I}=\sqrt{\frac{\pi}{2}} \frac{\omega k_{D}^{3}}{\omega_{p} k^{3}} e^{-\frac{m_{e} \omega^{2}}{k^{2} 2 T_{e}}},
\end{gathered}
$$

where $k_{D}$ is the Debye wavenumber

$$
k_{D}=\int_{-\infty}^{i n f t y} \sqrt{\frac{T_{e}}{4 \pi n_{e} e^{2}}} e^{-2 \pi i k x} d x .
$$

Here, $\omega_{p}$ is the plasma frequency $\sqrt{\frac{n_{e} e^{2}}{\epsilon_{0} m_{e}}}$ and $\phi(x)$ is Dawson's integral (83). An important point is that the width operator $G(\Delta \omega)$, to first order, increases linearly with the electron density $n_{e}$, as it was for PrismSPECT. Thus an increase in $n_{e}$ leads to $G(\Delta \omega)$ increasing as well. 


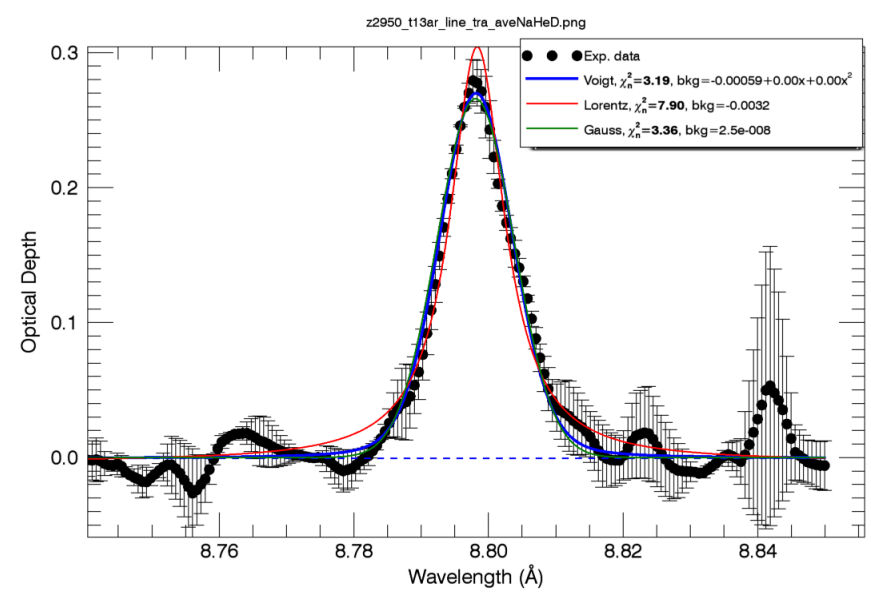

Figure 4.1: The fit for the $N a-H e \delta$ line from z2950. This process is the same as the one described in section 3.3. The width for this line was found to be $0.0129 \pm 0.0004 \AA$

\subsection{Fitting the Data}

Fitting the experimental data is handled in the same way as for chapter 3. The line transmission data is converted into optical depth, then fit with Voigt, Gaussian and Lorentzian profiles, with the Voigt taken as the best fit. The only difference is that the width is the important factor for this investigation. In fact, only He-like lines were required for this experiment, as they will be more affected by Stark broadening than lower charge state lines. Fortunately, a line need only be fit once to record the area and width. Some examples include figures 3.4, 3.5, 4.1 and 4.2 .

\subsection{PrismSPECT fitting}

The method for fitting PrismSPECT, much like fitting the data, was the same as that for chapter 3 . The PrismSPECT spectra was convolved with the appropriate instrument function, either axially resolved KAP or axially resolved TAP (in second order), and then converted into optical depth. One processing modification for this fitting was the change in PrismSPECT temperature and density 


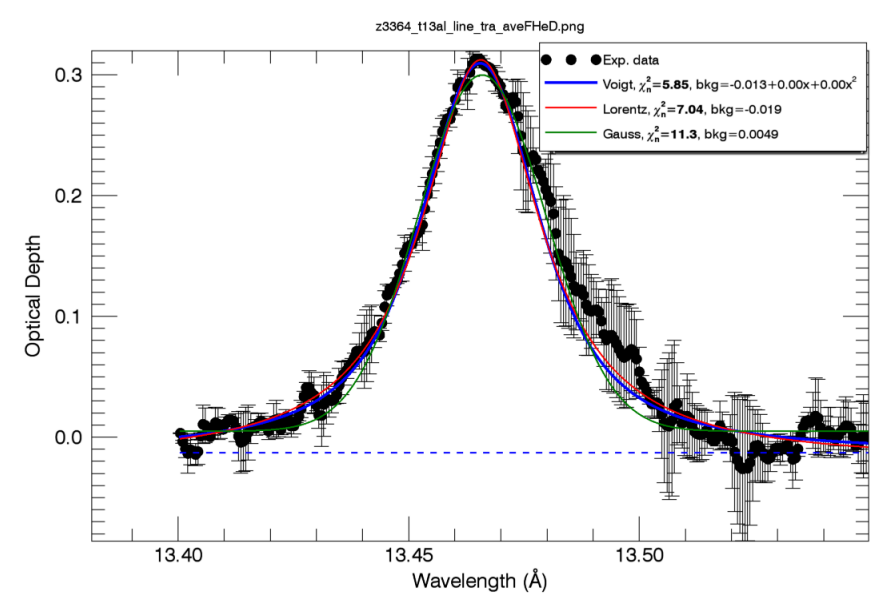

Figure 4.2: The fit for the $F-H e \delta$ line from z3364. The width of this line was determined to be $0.0312 \pm 0.0005 \AA$

inputs. The ATBASE files remained the same. Instead of the finer temperature and density grid, used for the isoelectronic line ratio study, a courser array was used. The ion densities ranged from $9 \times 10^{19} \mathrm{~cm}^{-3}$ to $1 \times 10^{21} \mathrm{~cm}^{-3}$ with a step of $1 \times 10^{19}$ followed by steps of $1 \times 10^{20} \mathrm{~cm}^{-3}$, and the temperatures ranged from $50 \mathrm{eV}$ to $75 \mathrm{eV}$ in steps of $2.5 \mathrm{eV}$. The increase in density range establishes the density sensitivity of the linewidths unambiguously without scaling up our computational time. The smaller range in temperature acknowledges we have an estimate of temperature from the inter-stage and isoelectronic line ratios.

Once a line was $\chi^{2}$ fit over all temperature and ion density combinations, the line widths could be plotted to inspect how the linewidths behave as a function of ion density according to PrismSPECT (figures 4.3 and 4.4). The width of a line varies linearly with the ion density. By fitting a line $(y=m x+b)$ to these points, a single equation can be found that would allow for conversion between a line's measured width and inferred ion density (figures 4.5 and 4.6). The assignment of y-intercept would benefit from fewer temperatures involved in the fit. Higher temperatures correlate with slightly larger widths relatively, because the Doppler width is temperature dependent.

Note that we are fitting with respect to ion density, as that is the adjustable parameter in PrismSPECT. However, what we will be quoting later is electron 


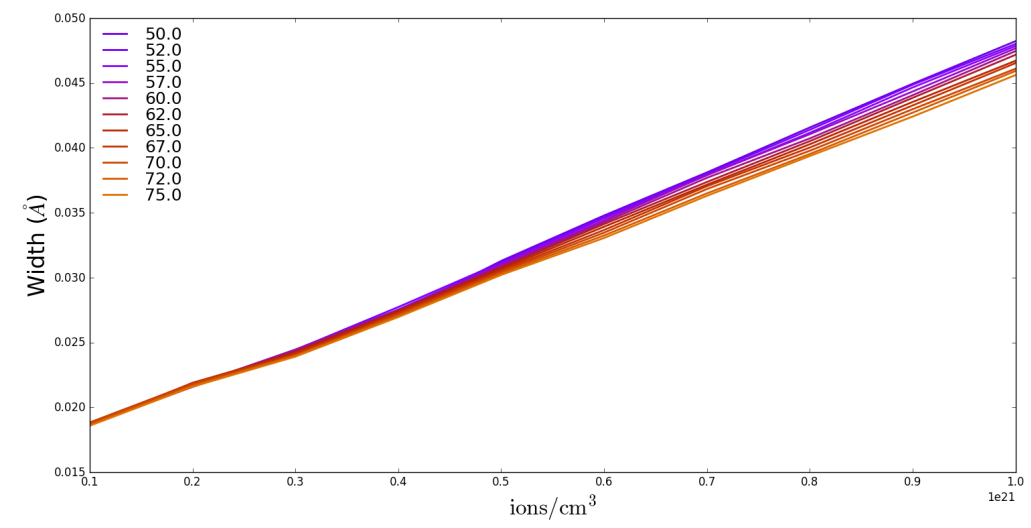

Figure 4.3: Fitting the $F-H e \gamma$ for all the temperatures and densities used in PrismSPECT, we can see a linear dependence on ion density.

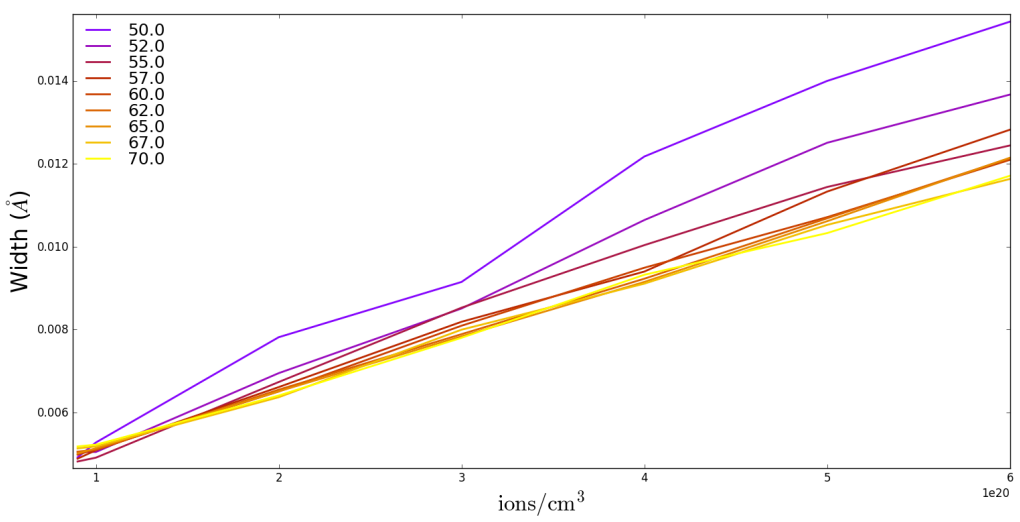

Figure 4.4: Because of a problem from our fitting routine some lines, such as the $M g-H e \gamma$, seem to have less linearity to their dependence on ion density than other lines. As temperatures approach $50 \mathrm{eV}$ this line starts to get very small, especially when broadening is factored in limiting an accurate fit. 


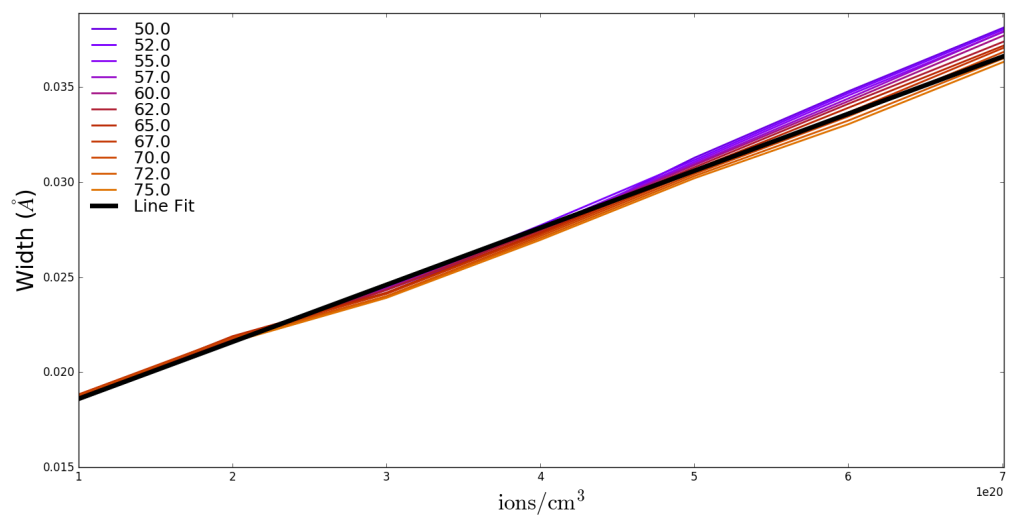

Figure 4.5: A linear function is fit to the width vs density graph. This allows for easy conversion from our measured width to an inferred density. Fitting with a $y=m x+b$, we find that $m=3.66 \times 10^{-23}\left(\frac{\AA}{\text { ions } / c c}\right)$ and $b=0.0127(\AA)$

density. In order to convert from ion density to electron density, we assume charge conservation, therefore we just need to multiply the ion density by the average charge state of the plasma (12)

$$
\begin{gathered}
n_{e}=n_{i} \bar{Z} \\
\bar{Z}=\frac{n_{M g} \overline{Z_{M g}}+n_{N a} \overline{Z_{N a}}+n_{F} \overline{Z_{F}}+n_{O} \overline{Z_{O}}}{n_{M g}+n_{N a}+n_{F}+n_{O}}
\end{gathered}
$$

where the average charge state $\bar{Z}$ is the weighted average of the average charge states of each of the constituent elements. It is important to include all of the elements found in RBS measurements of the experimental foils because Oxygen in the foil supplies a considerable number of electrons. PrismSPECT calculates the average charge state, which is found to be $7.00 \pm 0.15$.

An alternate way to convert width into electron density would be to plot the fitted widths versus the electron density for each temperature and density (figure 4.7). This method is more time consuming, as the electron density must be known for every temperature and density individually, but it allows for a more direct conversion to electron density from a measured width as opposed to calculating ion density and using $\bar{Z}$ as a conversion factor. When comparing the 


\section{STARK EFFECT ON MULTIPLE, MULTIPLY-IONIZED}

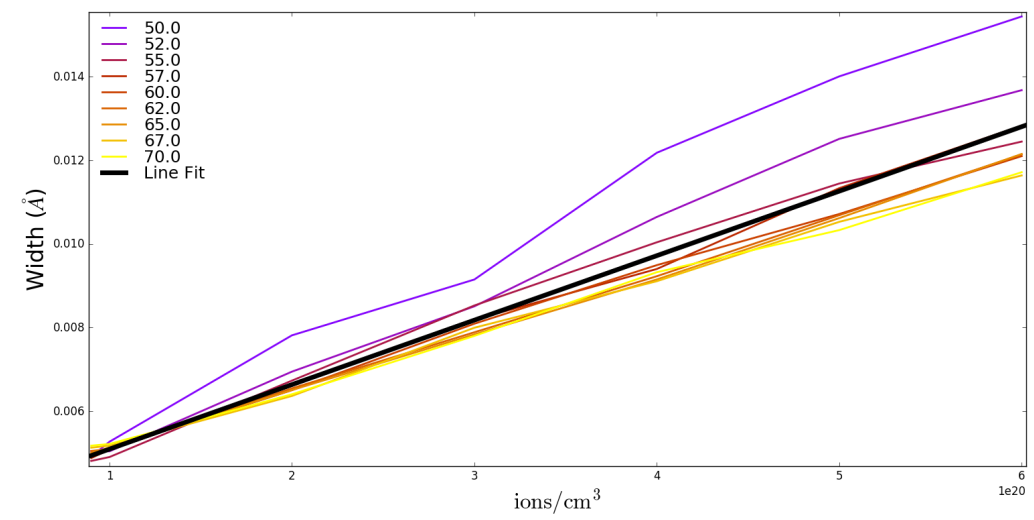

Figure 4.6: Fitting PrismSPECT lines can be applied to all the spectra of interest, including this $M g-H e \gamma$. While width vs density dependence is less linear at lower temperatures, we believe the fit is still reasonable. We found that $m=$ $1.54 \times 10^{-23}\left(\frac{\AA}{i \text { ions } / c c}\right)$ and $b=0.0036(\AA)$

two methods, both predict the same electron density within uncertainty. For the $M g-H e \gamma$ line measured on z3286, the $\bar{Z}$ method shown in figure 4.6 predicts $n_{e}=1.15 \times 10^{21} \pm 2.1 \times 10^{20}$, while the electron density method (figure 4.7) for the same line predicts $n_{e}=1.14 \times 10^{21} \pm 2.05 \times 10^{20}$.

\subsection{ATOMIC fitting}

The ATOMIC and PrismSPECT spectra are handled similarly. The data is convolved with the pertinent instrument functions, then converted to optical depth and fitted with Voigt profiles. Discrepancies arise from the different matrix of input plasma temperatures and densities used in ATOMIC. We used the temperatures $50,60,70 \mathrm{eV}$ and densities of $1 \times 10^{20}$ to $7 \times 10^{20}$ ions per cc, with steps of $1 \times 10^{20}$. This course grid still achieved the same range in temperatures, but did not ask too much of Dr. Fontes, who ran the code for us as it is proprietary to Los Alamos National Labs. This is the same reason we truncated the ion densities at $7 \times 10^{20}$, as preliminary results from our PrismSPECT analysis showed that our densities were nowhere near that high.

As with the PrismSPECT data, each spectral line is fit for every combination 


\subsection{ATOMIC fitting}

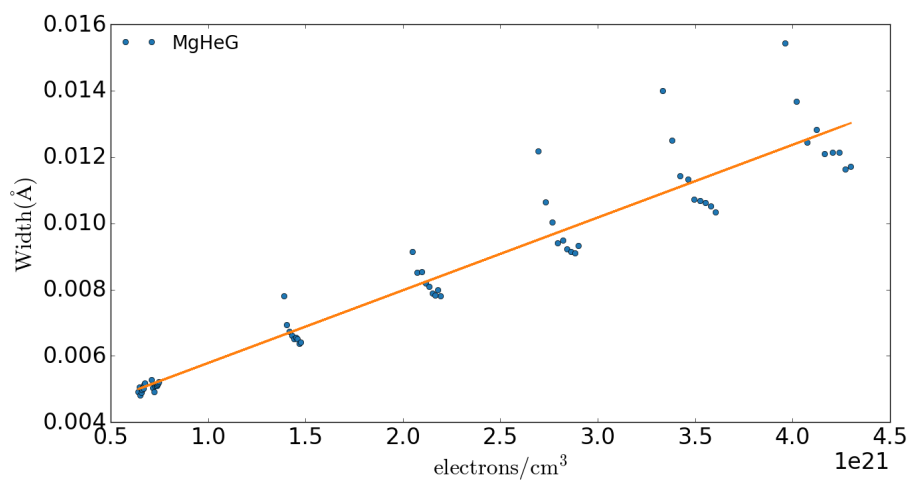

Figure 4.7: PrismSPECT can also output the electron densities, taking into account both temperature and ion density. This can be fit to provide a direct comparison of width and electron density. This method and fitting for ion density and converting using $\bar{Z}$ agree within error when determining $n_{e}$.

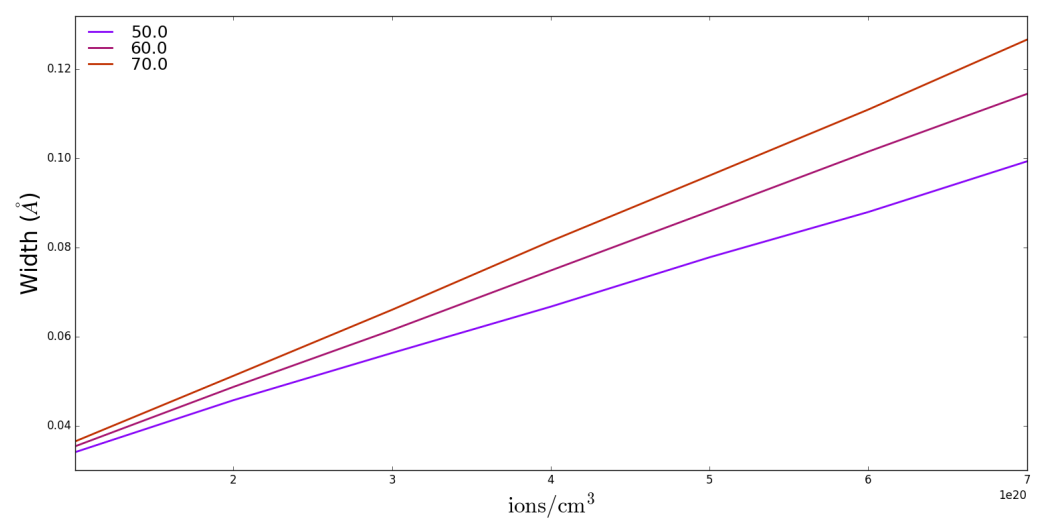

Figure 4.8: Fitting the $F-H e \delta$ for all temperatures and ion densities from ATOMIC, we then plot the width versus the ion density. The width appears to have a linear dependence on ion density, which holds true for most of the He-like lines we're interested in. 


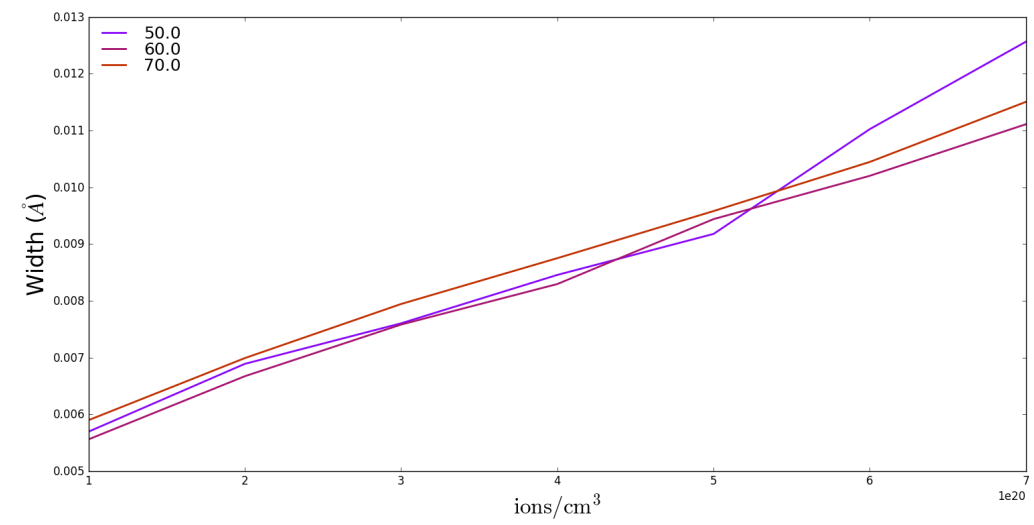

Figure 4.9: Fitting the $M g-H e \gamma$ for all temperatures and ion densities from ATOMIC. Lines below $8 \AA$ were only looked at using the TAP instrument resolution, as the KAP was not accurate enough, as shown by figure 2.13.

of temperature and density, and the resultant widths are plotted versus the input ion density, as seen in figures 4.8 and 4.9. These plots indicate that our ATOMIC widths also have a linear dependence on ion density. Some of the line widths deviate from the best line fit, such as the $M g-H e \gamma$ (figure 4.9), but the deviations in width are small enough (smaller than a $m \AA$ ) retain the credulity of the linear fit.

The same linear fitting technique is applied to the ATOMIC results as was applied to the PrismSPECT results. This linear conversion between measured width and ion density converts to electron density using the method described above (figures 4.10 and 4.11). Note that ATOMIC does not output the average charge state or electron density like PrismSPECT does. Instead, we use the average charge state found in PrismSPECT, as the spectra from both agree and because the He-like charge state (the predominant charge state for Fluorine and Oxygen and Sodium and a large part of Magnesium) is a slowly varying one with respect to temperature, so the assumption of $\bar{Z}=7$ is a good one. 


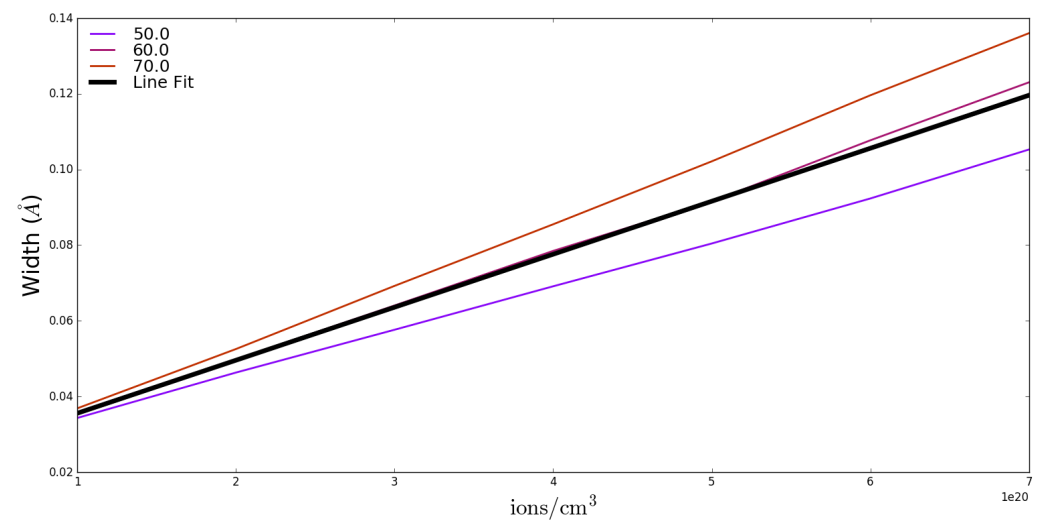

Figure 4.10: By fitting a linear function $(y=m x+b)$ to the width vs ion density graph, it becomes possible to easily convert our measured width (y) into ion density (x). This is the $F-H e \delta$ fit from ATOMIC, with $m=1.42 \times 10^{-22}$ and $b=0.022$.

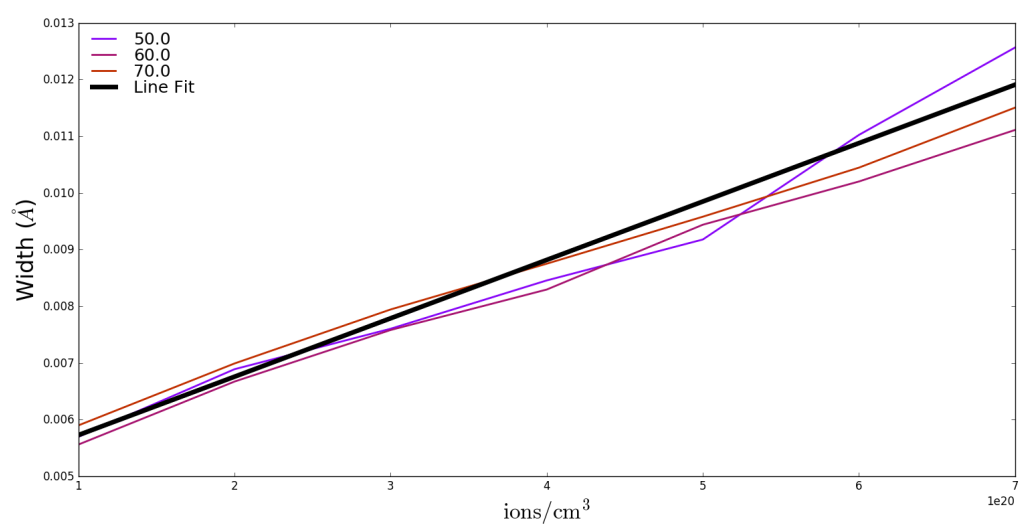

Figure 4.11: Fitting the ATOMIC widths with a linear function is repeated for all of the lines of interest, such as the $\mathrm{Mg}-\mathrm{He \gamma}$, where it was found $m=1.03 \times 10^{-23}$ and $b=0.0048$. 


\subsection{Analysis}

Once a linear function is found, such that width $=m *$ density $+b$ is found, we can invert this to find density and its uncertainty when given a width and its uncertainty. We assume that our width is prescribed by a Gaussian distribution, with the centroid as the measured value of the line's width, and the standard deviation of the fit's width as the uncertainty in the width found from our fit. Doing so will allow us to visualize the probability of electron density as a Gaussian distribution. This in turn lets us see how much overlap these probabilities associated with different lines have in density space, which makes for a better interpretation of agreement.

Inverting our equation width $=m *$ density $+b$ gives us the linear transform of density $=($ width $-b) / m$, which allows us to convert our width uncertainty Gaussian into an ion density Gaussian. It should be noted that in doing so we need to propagate the errors from both the width measurement and the linear fit, as both $m$ and $b$ contribute to overall variance. This can be implemented by using the equation for error propagation

$$
\sigma_{F}=\sqrt{\sum_{i}\left(\frac{\partial F}{\partial x_{i}}\right)^{2} \sigma_{x_{i}}^{2}}
$$

By plugging in our equation for width into this formula, we find that the error in density is given by

$$
\begin{gathered}
\sigma_{\text {density }}=\sqrt{\left(\frac{\partial \text { density }}{\partial w i d t h}\right)^{2} \sigma_{\text {width }}^{2}+\left(\frac{\partial d e n s i t y}{\partial b}\right)^{2} \sigma_{b}^{2}+\left(\frac{\partial d e n s i t y}{\partial m}\right)^{2} \sigma_{m}^{2}} \\
\sigma_{\text {density }}=\sqrt{\left(\frac{1}{m}\right)^{2} \sigma_{\text {width }}^{2}+\left(\frac{-1}{m}\right)^{2} \sigma_{b}^{2}+\left(\frac{w i d t h-b}{m^{2}}\right)^{2} \sigma_{m}^{2}}
\end{gathered}
$$

When converting from width space into ion density space it is important to broaden the Gaussian by using this total uncertainty, as opposed to just the width uncertainty. The Gaussian ion density distribution is then multiplied by the mean charge state of the plasma, $\bar{Z}=7$, converting the ion density into electron density. This process is then applied to all the relevant lines from relevant 


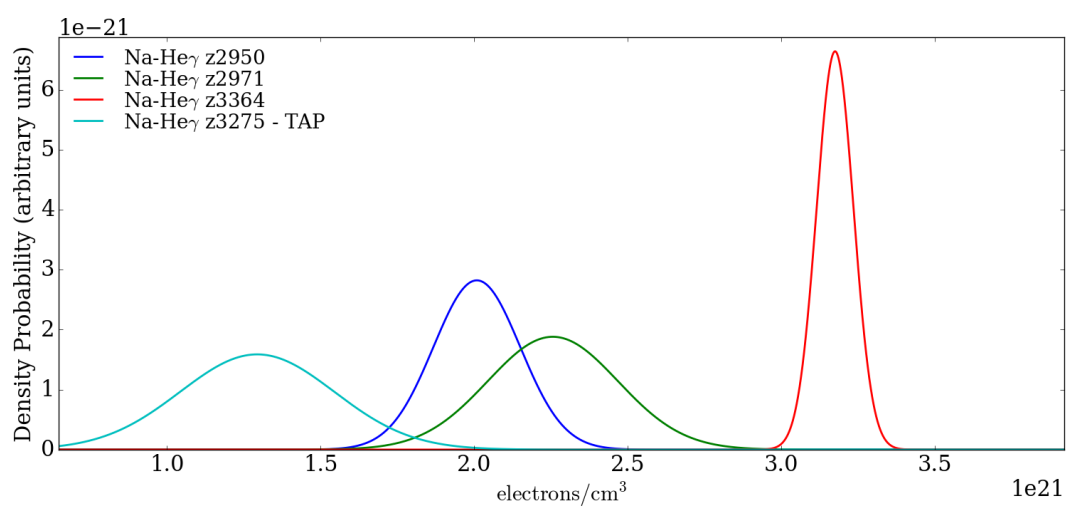

Figure 4.12: The results of the linear conversion of the Gaussians for the $\mathrm{Na}-\mathrm{He \gamma}$ line for all of the $4 \mu \mathrm{m}$ shots. The linear conversion used was from PrismSPECT. Deviations between curves implies shot-to-shot variations.

shots. It should be noted that Stark broadening more heavily affects higher $\mathrm{n}$ transitions (transitions going into or leaving states with higher principle quantum numbers), so the $N a-H e \alpha, M g-H e \alpha$ and the $F-H e \beta$ were not included in this analysis. The $\mathrm{Na}-\mathrm{He} \beta$ was not included as it lies directly on top of the $\mathrm{Mg}-\mathrm{Be}$ features, and thus a good fit is improbable. Results for $\mathrm{Na}-\mathrm{He \gamma}$ line for the $4 \mu m$ case and the $M g-H e \gamma$ line for the $7 \mu m$ case can be seen in figures $4.12,4.13,4.14$ and 4.15 .

\subsection{Results}

Once every appropriate line has been fit, we can compare the results across different elements to assess consistency. To help make this easier, as well as to maintain consistency with the isoelectronic line ratio technique, we average the results from the same tamper, same line together. This allows us display a single Gaussian from each line to compare with during the line-to-line comparison. In order account properly for the relative contributions of each spectral line, we use 


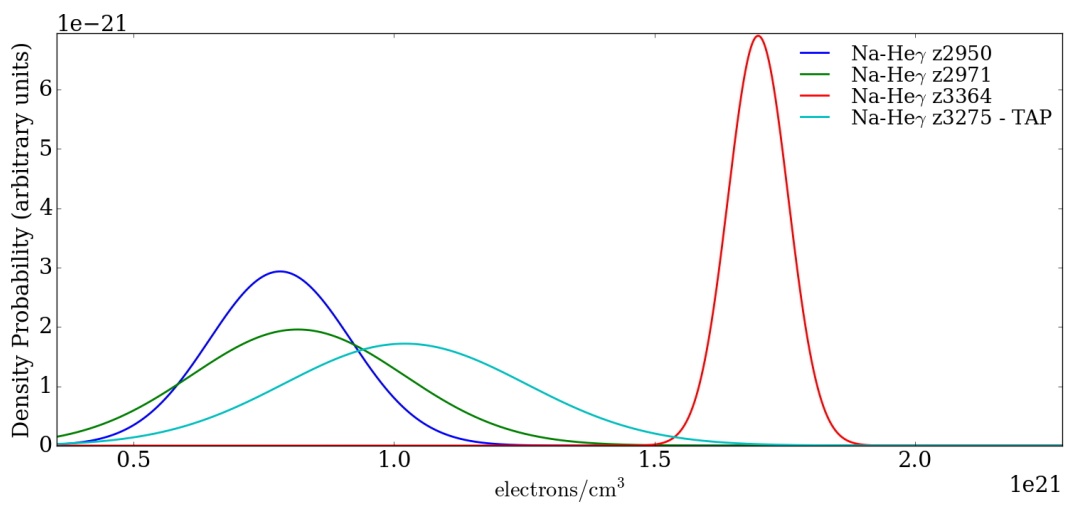

Figure 4.13: The results of the linear conversion of the Gaussians for the $\mathrm{Na}-\mathrm{Her}$ line for all of the $4 \mu \mathrm{m}$ shots. The linear conversion used was from ATOMIC. In general, ATOMIC results skew to lower densities.

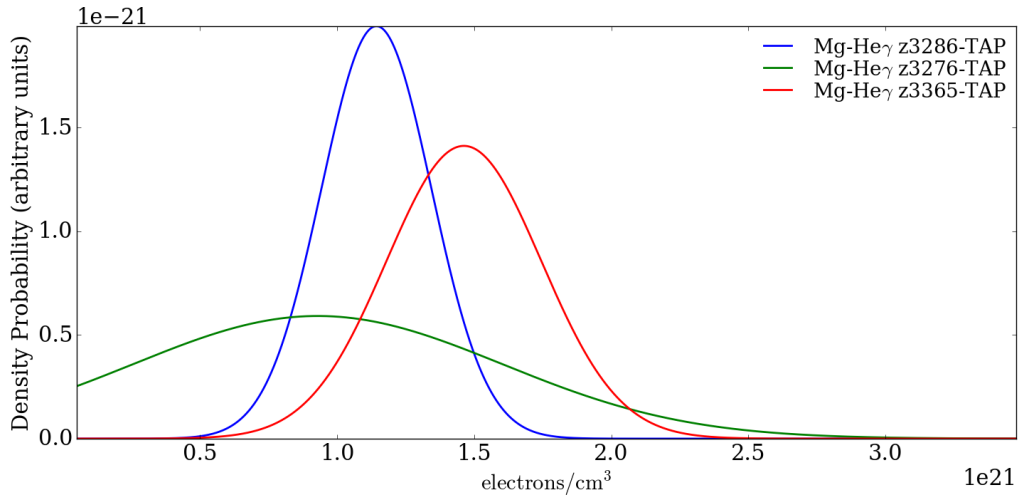

Figure 4.14: A similar plot, but for the $M g-H e \gamma$ lines from $7 \mu m$ experiments using PrismSPECT. 


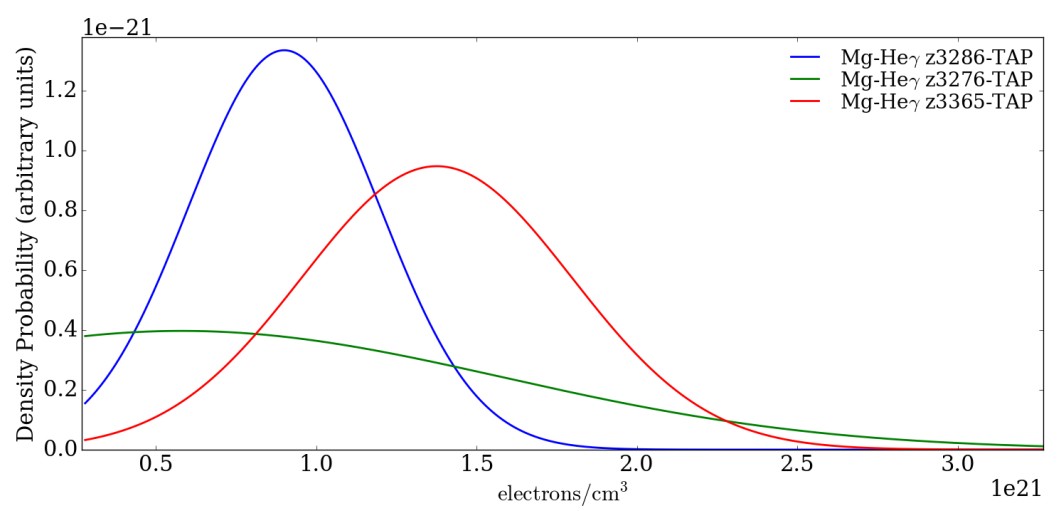

Figure 4.15: The $7 \mu m, M g-H e \gamma$ data interpreted using ATOMIC results. Once again we see that ATOMIC predicts lower densities. Deviations between curves implies shot-to-shot variations.

a weighted average given by

$$
<n_{e, \text { line }}>=\frac{\sum_{i=1}^{n} \frac{1}{\sigma_{i, \text { Line }}} n_{e, i, \text { Line }}}{\sum_{i=1}^{n} \frac{1}{\sigma_{i, \text { Line }}}},
$$

and the standard deviation for this average, given by

$$
\left(\frac{\sigma_{\text {avg }}}{<n_{e, \text { Line }}>}\right)^{2}=\sum_{i=1}^{n}\left(\frac{\sigma_{i, \text { Line }}}{n_{e, i, \text { Line }}}\right)^{2} .
$$

It should be noted that this is the same way the individual shot results were averaged into configuration results with standard deviation that was used for the isoelectronic and inter-stage line ratios.

\subsubsection{4 micron Tamped}

The results for the $4 \mu m$ tamped case using PrismSPECT are found in figure 4.16. The $\mathrm{Mg}-\mathrm{He \gamma}, \mathrm{Na}-\mathrm{He} \delta$ and $\mathrm{Na}-\mathrm{He \epsilon}$ lines do not agree with the He-like F lines nor the other $\mathrm{Na}$ and $\mathrm{Mg}$ lines, but they do agree with each other, and all three predict a lower density than the other lines. This indicates that these lines may not be suited for cases with slightly lower densities, for example electron densities of around $2.5 \times 10^{21} \mathrm{~cm}^{-3}$ as predicted by the other lines. 


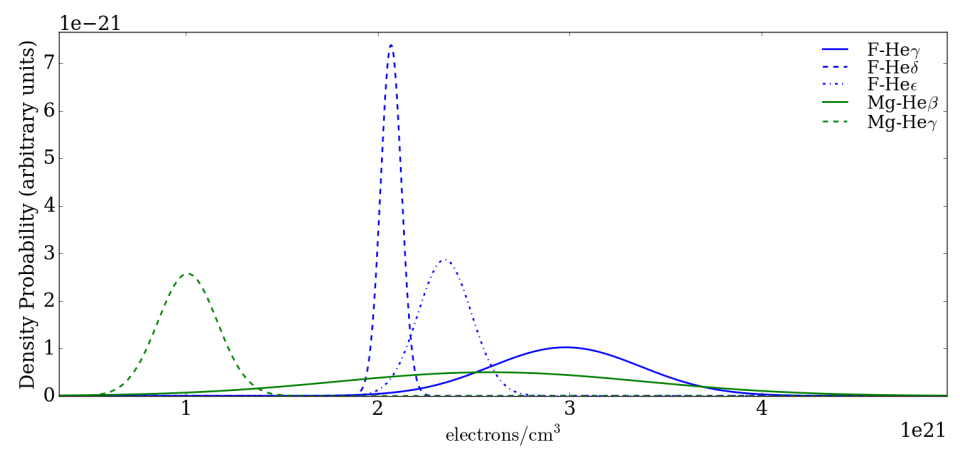

(a) The density predicted by the He-like F lines compared to the He-like Mg lines.

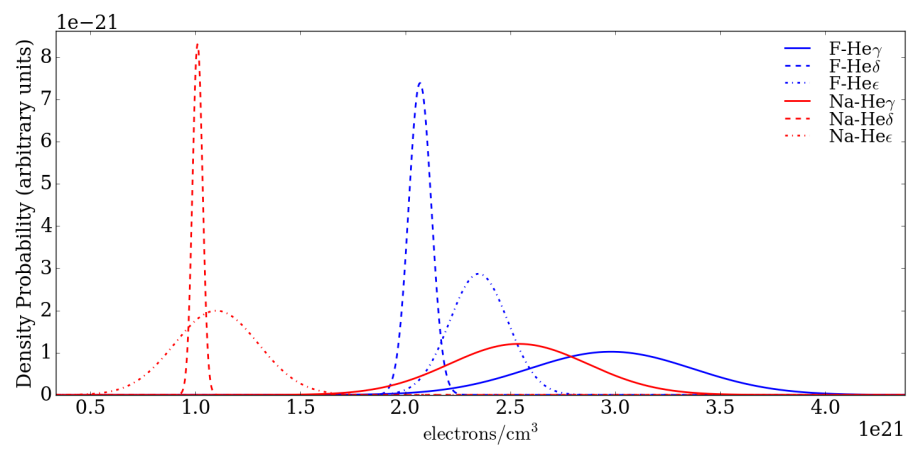

(b) The density predicted by the He-like F lines compared to the He-like Na lines.

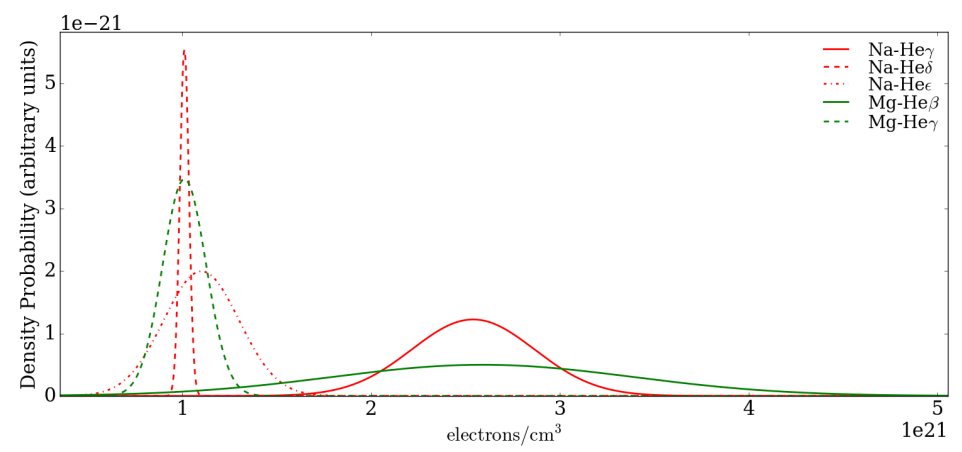

(c) The density predicted by the He-like Na lines compared to the He-like Mg lines.

Figure 4.16: The average densities predicted by each He-like line for the $4 \mu \mathrm{m}$ case based on PrismSPECT calculations are compared across elements. 
The ATOMIC results are in figure 4.17. The first thing to note is that ATOMIC predicts a lower density than PrismSPECT. Because of this, there is a natural "bunching" of the predicted electron densities, making them closer to each other. This leads to the natural conclusion that these lines agree under these conditions. It should be noted that some individual lines do not agree with each other, such as the $\mathrm{F}-\mathrm{He \epsilon}$ and the $\mathrm{Na}-\mathrm{He \gamma}$, but we are more interested in the complex of lines coming from one element and how that compares to another element. Within this frame, the different elements agree.

\subsubsection{7 micron Tamped}

From the $7 \mu m$ tamped PrismSPECT results (figure 4.18), the first thing to notice is that the predicted electron densities for every line are higher than with the $4 \mu \mathrm{m}$ tamping. This makes sense, as we've seen that a higher tamper thickness leads to a lower temperature, which would result in less expansion of the sample. Within this sample, we see that once again the higher $\mathrm{n}$ transitions of $\mathrm{Mg}$ and $\mathrm{Na}$ do not agree with the He-like F lines. The $M g-H e \delta, M g-H e \gamma, N a-H e \delta$ and $\mathrm{Na}-\mathrm{He \epsilon}$ agree with each other, and to a certain extent the $\mathrm{Mg}-\mathrm{He} \beta$.

Something of concern is how the $\mathrm{Na}-\mathrm{He \gamma}$ has not agreed with the other He-like Na lines based on PrismSPECT results for either tamper thickness. If this line were taken out of the analysis then it would imply that the He-like F lines do not agree with the $\mathrm{Na}$ or $\mathrm{Mg}$ lines, predicting a higher temperature than lines from the other elements.

As with PrismSPECT, ATOMIC predicts higher densities on average, but not for every line. The $M g-H e \beta$ and $M g-H e \gamma$ predict approximately the same densities that they predicted in the $4 \mu \mathrm{m}$ case. However, these lines still agree with the lines from He-like F and most of the He-like Na. The one line that is out of place is the $\mathrm{Na}-\mathrm{He \gamma}$ which predicts a much higher density than all of the other lines. This implies that the $\mathrm{Na}-\mathrm{He \gamma}$ line is not a good line for determining density, as both PrismSPECT and ATOMIC show it disagreeing at higher density, with PrismSPECT showing disagreement at lower densities as well. Aside from the $\mathrm{Na}-\mathrm{He \gamma}$, the lines show agreement on electron density based on ATOMIC calculations. 


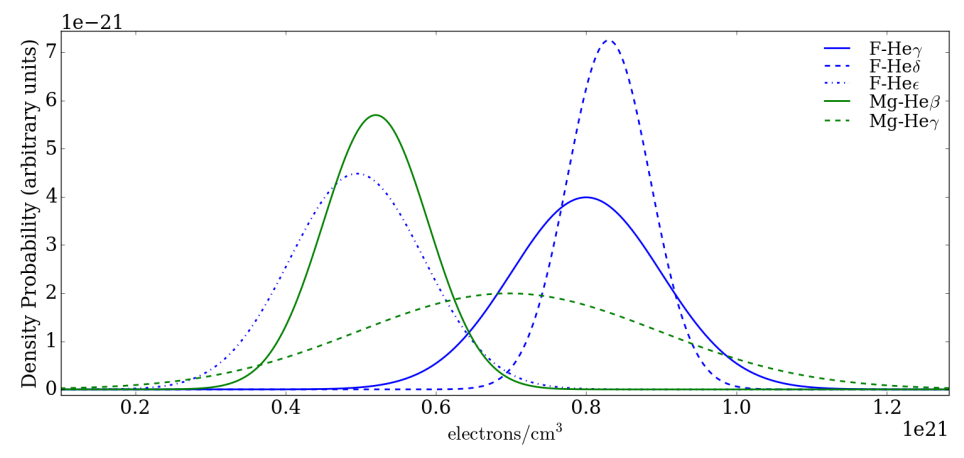

(a) The density predicted by the He-like F lines compared to the He-like Mg lines.

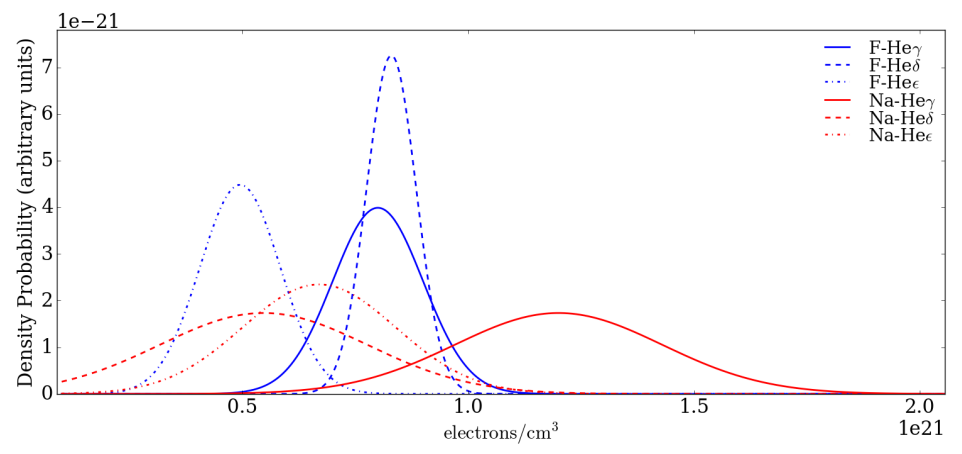

(b) The density predicted by the He-like F lines compared to the He-like Na lines.

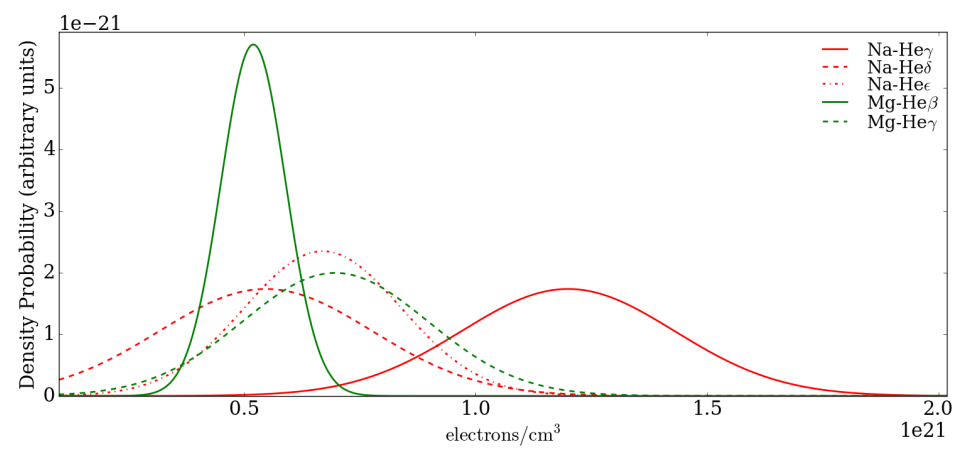

(c) The density predicted by the He-like Na lines compared to the He-like Mg lines.

Figure 4.17: The average densities predicted by each He-like line for the $4 \mu \mathrm{m}$ case based on ATOMIC calculations are compared across elements. 


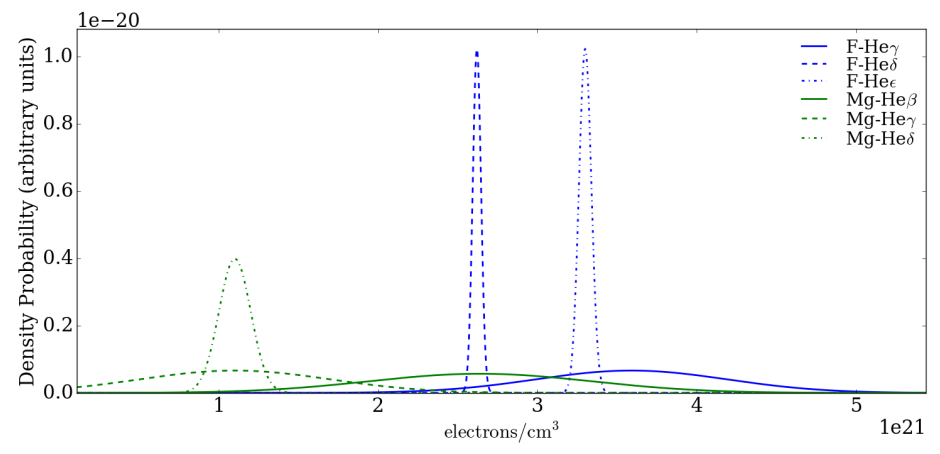

(a) The density predicted by the He-like F lines compared to the He-like Mg lines.

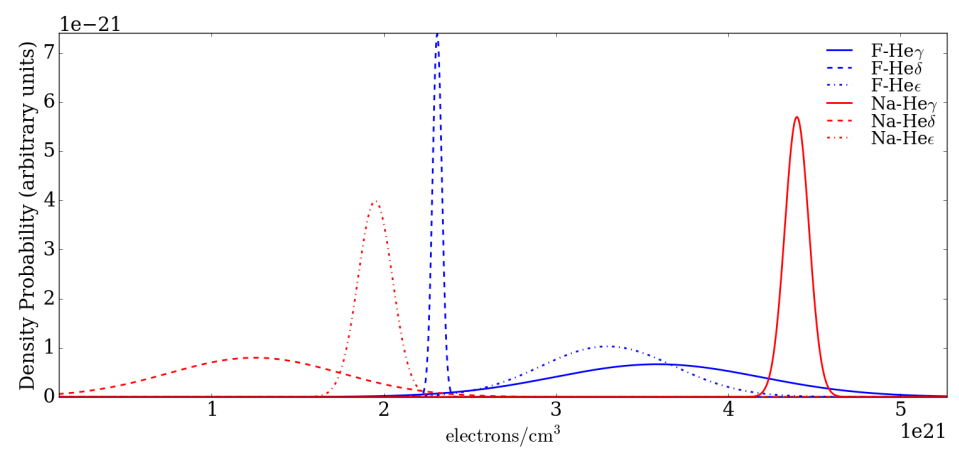

(b) The density predicted by the He-like F lines compared to the He-like Na lines.

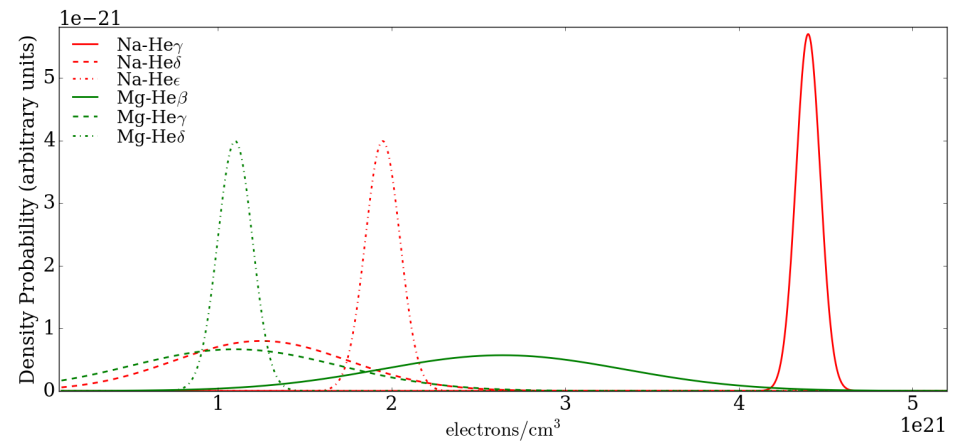

(c) The density predicted by the He-like $\mathrm{Na}$ lines compared to the He-like Mg lines.

Figure 4.18: The average densities predicted by each He-like line for the $7 \mu m$ case based on PrismSPECT calculations are compared across elements. 


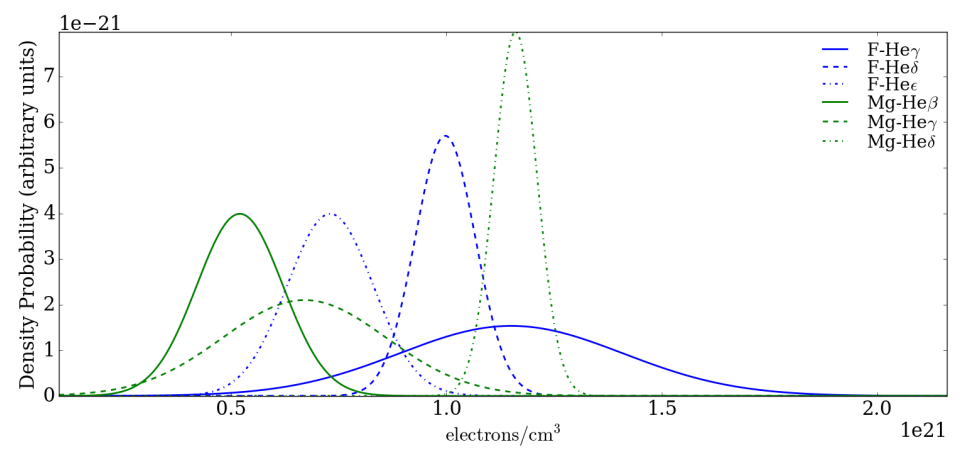

(a) The density predicted by the He-like F lines compared to the He-like Mg lines.

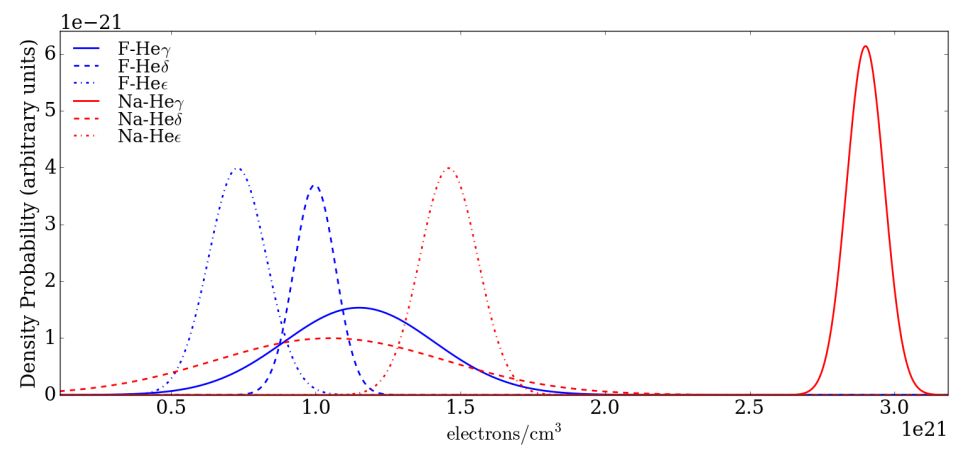

(b) The density predicted by the He-like F lines compared to the He-like Na lines.

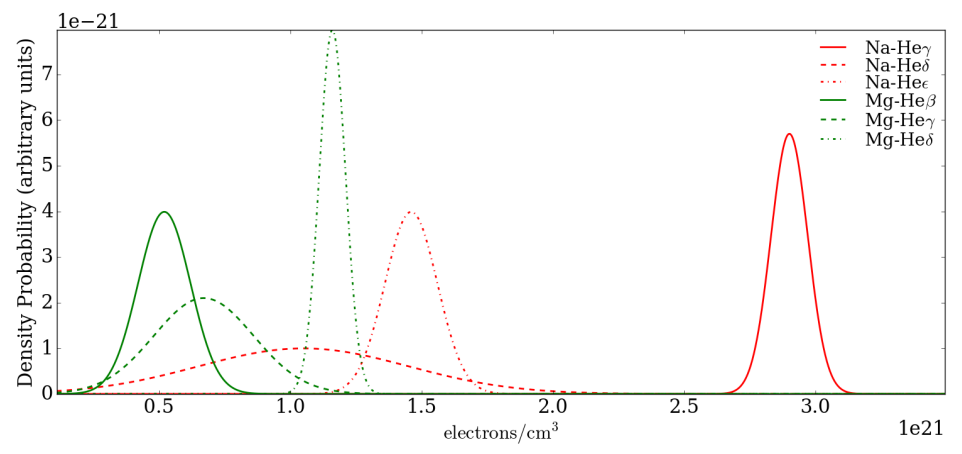

(c) The density predicted by the He-like Na lines compared to the He-like Mg lines.

Figure 4.19: The average densities predicted by each He-like line for the $7 \mu \mathrm{m}$ case based on ATOMIC calculations are compared across elements. 


\subsubsection{Causes of Discrepancies}

When looking into equation 4.1 two possible causes of discrepancies in inferred electron density are the electric microfield probability $P(\epsilon)$ and the electron broadened profile $J(\omega, \epsilon)$. We can rule out the electric microfield for discrepancies within the same code, as within MERL or ATOMIC, the electric microfield probability is going to be the same for all ions in a homogeneous plasma, which we assume our plasma is.

In the MERL formalism for $J(\omega, \epsilon)$ which PrismSPECT uses, there are two possible causes for discrepancies across lines and elements, the radiator dipole operator $d_{i, f}$ and the electron broadening operator $M(\omega)_{i f, i^{\prime} f^{\prime}}$. We converge on this because in equation 4.6 everything else is well defined for all lines, such as the Liouville operator. It is unlikely that $d_{i, f}$ is the cause of problems, as it is defined as $\left\langle\Psi_{a}|q \vec{r}| \Psi_{b}>\right.$ in quantum mechanics, so the error would lie in the position vector. It is more likely that the issue arises from $M(\omega)_{i f, i^{\prime} f^{\prime}}$, more specifically the function $G(\Delta \omega)$ in equation 4.9. This function relies on Gaunt factors (74) (84), which are approximations that may not be accurate for all lines.

In the Lee model which ATOMIC uses, the possible culprits for the cause of disparate electron densities from linewidths is either the radiator dipole moment or the width operator, $G(\Delta \omega)$. This is seen in equation 4.12 , where only $d_{i, f}$ and $G(\Delta \omega)$ are not constants. As with MERL, it is unlikely that the radiator dipole moment is the problem, leaving $G(\Delta \omega)$ as the more likely origin of discrepancies in ATOMIC results. We see in equation 4.13 and equation 4.14 that much of what compromises $G(\Delta \omega)$ are constants and $k$ which is integrated over. As discussed in (80), the integral in equation 4.13 is often evaluated for only small or large $\Delta \omega$ values, leaving intermediate values out so that computational speed is maximized. This is most likely the origin of discrepancies in ATOMIC results.

The lack of agreement between PrismSPECT and ATOMIC results, belies a consistency problem (ATOMIC being around thirty to fifty percent of what PrismSPECT predicts). These models employ different electric microfield probability models and employ different electron broadened profiles, both of which may contribute to the lack of agreement. An interesting test would be to incorporate the APEX model for microfield probabilities that ATOMIC uses with the 
electron broadened profiles from MERL, which would determine how much the profiles matter when comparing across codes as opposed to just the microfield probabilities. In the next chapter we will discuss possible experimental errors whose investigation would require further experiments. One candidate for explaining these discrepancies is the assumption of a homogeneous plasma. 


\section{5}

\section{Summary, Conclusions and Future Work}

Physics is really nothing more than a search for ultimate simplicity, but so far all we have is a kind of elegant messiness.

-Bill Bryson

\section{$5.1 \quad$ Summary}

In this thesis, we investigated two spectroscopic techniques: whether the technique of isoelectronic line ratios is a valid temperature diagnostic in absorption spectra, and if the technique of predicting electron density through Stark broadening agrees when inferred from different elements. In order to investigate these techniques, experiments were planned and executed at Sandia National Labs' Z machine in New Mexico, using facility ride-along opportunities associated with fundamental and programmatic science campaigns. A previously established setup using the Z Pinch Dynamic Hohlraum (ZPDH) provided a platform for fielding multiple separate experiments, including our own. Radiation from the ZPDH was used to heat and backlight our experiment's target foil. The experiments used thin foils ( $\mu \mathrm{m}$ scale) comprised of $\mathrm{MgO}_{2}-\mathrm{NaF}$ that were tamped with various amounts of $C_{6} H_{6}$ to manipulate the temperature and density of the foil plasma. The foils were attached directly to the Return Current Can of the Z pinch so as to maximize the amount of radiation incident on the foil without affecting 


\section{SUMMARY, CONCLUSIONS AND FUTURE WORK}

the symmetry of the pinch implosion. This location for the foils allowed for higher temperatures to be achieved. In order to measure spectra, the Time Integrated Crystal Spectrometer (TIXTL) was fielded on Z, using both a four-inch Potassium Acid-Phthalate (KAP) and a six-inch Thallium Acid-Phthalate (TAP) crystal. Fourteen viable spectra, collected over a series of ten shots on Z, served as the raw experimental data. Tamper thickness was either 4,7 or $15 \mu \mathrm{m}$. The transmission data was processed to go from film intensity and position on film to line transmission and wavelength. The $\mathrm{Z}$ facility provided and mounted the target foils and the ZPDH, as well as digitized the film. Prism CS supplied the spectral simulation codes. LANL provided the simulation results from ATOMIC. We supplied manpower to calibrate and process data. We created analysis routines and codes. We ran PrismSPECT and HELIOS, and carried out the data interpretation.

\subsection{Conclusions}

Chapter 3 described the process of investigating isoelectronic line ratios in absorption spectra. This was done by converting line transmission spectra into optical depth, then fitting spectral lines with Voigt profiles in order to extract the area of the spectral line. This process was applied to almost every line in every spectra that was collected. Line areas from the same charge state, but originating from different elements were compared in a ratio, providing an isoelectronic line ratio value. Ratio values were also created by comparing line areas from different charge states, but from the same type of element, known as an inter-stage ratio which is a commonly used diagnostic currently. To get a temperature from these line ratios, synthetic spectra were created using the code PrismSPECT, which is a collisional-radiative model that can take in to account relative abundances of elements, as well as the atomic kinetics needed to construct spectra. A wide range of temperatures was input into PrismSPECT. The resulting synthetic transmission spectra were then convolved with our instrument function, ensuring representative spectra, then converted into optical depth. Individual spectral lines were then fit with Voigt profiles. Both inter-stage and isoelectronic line ratio values from experiments were compared with values from PrismSPECT to infer 


\subsection{Conclusions}

temperature values for the three tamper cases. Comparing the isoelectronic results to the inter-stage results, we saw that there was agreement between the two methods for all tamper thicknesses; the $4 \mu \mathrm{m}$ tamped case having $57 \pm 3.5 \mathrm{eV}$ from the isoelectronic ratios and $59.9 \pm 2.6 \mathrm{eV}$ for the inter-stage results. It should be noted that for all tamper thicknesses, inter-stage line ratios predicted a slightly larger value for temperature than the isoelectronic line ratios, this may be due to a temperature gradient in the sample of temporal evolution during the 3ns backlighter.

Chapter 4 concerned the investigation into Stark broadening. To investigate Stark broadening, the fitted Voigt profiles for He-like ions were used, but instead of areas, the widths of lines were evaluated. Natural broadening and Doppler broadening were found to be too small to impact the widths we documented. In order to determine electron density from these widths, the codes PrismSPECT and ATOMIC were used to create synthetic spectra. ATOMIC was added to see how a different $\mathrm{CR}$ code would predict electron density, and to see if both of the codes were consistent. ATOMIC is a CR code from Los Alamos National Lab that Dr. Chris Fontes ran for us. The ATOMIC and PrismSPECT data were convolved with our instrument functions, and the relevant He-like lines were fit. Looking at the fits as a function of density, a linear trend became apparent. The widths as a function of density were fit to a $y=m x+b$ model, allowing for easy conversion between experimental width and ion density. Ion density was converted into electron density by multiplying by the mean charge state of the plasma, which was given as 7 . Once all lines were in electron density, the values from different shots, but with the same shot configuration and same line were averaged. These average values were compared to each other in the form of Gaussian error distributions to see how much they agreed with values from other elements. By comparing the results in this way, we found that for the $4 \mu m$ case, the $M g-H e \gamma, N a-H e \delta$ and $N a-H e \epsilon$ do not agree with the Helike F lines, nor the $M g-H e \beta$ and the $N a-H e \gamma$, predicting a lower density than the rest, resulting in two electron densities depending on which lines are used; approximately $1.05 \times 10^{21} \pm 2.5 \times 10^{20}$ for the $\mathrm{Mg}-\mathrm{He \gamma}, \mathrm{Na}-\mathrm{He} \delta$ and $\mathrm{Na}-\mathrm{He \epsilon}$ and approximately $2.45 \times 10^{21} \pm 4.5 \times 10^{20}$ for the He-like F lines and the remaining He-like $\mathrm{Na}$ and $\mathrm{Mg}$ lines. The electron densities predicted 


\section{SUMMARY, CONCLUSIONS AND FUTURE WORK}

by ATOMIC all agree, although the density predicted is significantly lower than that predicted by PrismSPECT, with ATOMIC predictions of approximately $7 \times$ $10^{20} \pm 2 \times 10^{20}$ electrons/cc. In the $7 \mu m$ case, the $N a-H e \gamma$ predicted much a higher electron density than the other lines, approximately $4.4 \times 10^{21} \pm 8 \times 10^{19}$, and should be discarded. The remaining He-like $\mathrm{Na}$ and $\mathrm{Mg}$ lines agree with each other, predicting electron densities of $1.5 \times 10^{21} \pm 6 \times 10^{20}$, and the He-like $\mathrm{F}$ lines agree with the $M g-H e \beta$, predicting $2.9 \times 10^{21} \pm 5.5 \times 10^{20}$ electrons/cc. In ATOMIC, all of the lines agree except for the $\mathrm{Na}-\mathrm{He \gamma}$, which predicts still predicted a much higher density than all the other lines, around $3 \times 10^{21} \pm 7 \times 10^{19}$ compared with $1 \times 10^{21} \pm 4 \times 10^{20}$ predicted by the remaining lines. Overall, the model used in ATOMIC seems to predict more consistently across elements than PrismSPECT, and the densities inferred using ATOMIC do not agree with those from PrismSPECT.

The differences in inferred density from within the same code must come from $J(\omega, \epsilon)$, the electron broadened profile in equation 4.1. This is because within MERL or ATOMIC, the electric microfield probability, $P(\epsilon)$ is going to be the same for all ions in a homogeneous plasma, where $J(\omega, \epsilon)$ is different based on which spectral line it is for. Within MERL (and thus PrismSPECT), the cause of these discrepancies is either the radiator dipole operator, $d_{i, f}$ or the electron broadening operator, $M(\omega)_{i f, i^{\prime} f^{\prime}}$. For ATOMIC, the discrepancies would be caused by differences in the radiator dipole operator for each ion in an element or the integral within the width function, $G(\Delta \omega)$, as the limits of this integral are often truncated for computational speed.

The differences in inferred electron density may also be due to experimental error. Below we will discuss some possible explanations, but it is our belief that the largest contribution of experimental error is our low sample size. We only have 2-3 spectra for which we can average our results over, and there is a distinct probability that they are sampling from different points along our Gaussian distribution. The only way to test this would be to conduct more shots on Z. While we are members of the ZAPP collaboration, we do not have any scheduled shots within the foreseeable future. 


\subsection{Future Work}

\subsubsection{Investigation of more Codes}

One of the first things to be done if this work is to be continued is to investigate how other CR codes take into account Stark broadening, and whether those models can produce agreeable results from across multiple elements. This could be done in the same way we incorporated PrismSPECT and ATOMIC for consistency, or the experimental data could be given to the lineshape modelers for them to use as a check on their codes. The first check of this could be done using FLYCHK (85), which is a free code that uses NIST databases of rate coefficients to produce spectra. FLYCHK does not use a detailed model past Li-like ions, but this should not pose a significant problem as we are looking into He-like ions. Other codes that would be interesting to involve include NOMAD (86), which Yuri Ralchenko may be willing to run for us, and SCRAM (87), which we could run at Sandia if given more time. The most interesting model would be XENOMORPH (88), developed by Thomas Gomez recently, which uses a full quantum calculation and extends beyond dipole approximations to calculate Stark Broadening. Involving more codes would also allow for a narrowing down of electron density range, as currently ATOMIC and PrismSPECT predict wildly different densities, having a third code that aligns with one of them would help determine which one is closer to the true value.

\subsubsection{Density Gradients}

One factor that could greatly impact our results in investigating lines widths with respect to Stark broadening is if there is a density gradient in our line of sight. Ideally this would be investigated by doing a full Radiation-Hydrodynamics simulation of our experiment, then modeling the resultant spectra and seeing how it may affect line widths.

As a first order test, we split the plasma into two sections, a section closer to the pinch with a lower density and a section away from the pinch with a higher density (figure 5.1); each section has half of the areal density of our samples. In 


\section{SUMMARY, CONCLUSIONS AND FUTURE WORK}

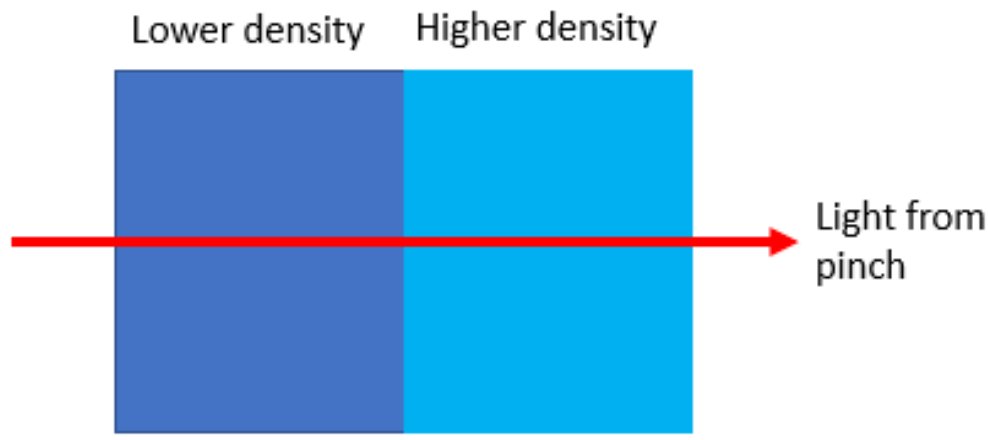

Figure 5.1: A schematic of how our simple, two-density gradient is set up. Half of the sample is at a lower density and half is at a higher density. The two emergent spectra are then multiplied together to reach a single transmission spectra.

this way, transmission spectra through both halves of this simulated plasma can create one transmission spectra using

$$
T_{\text {total }}=T_{1} T_{2},
$$

where $T_{x}$ is the transmission through part $x$ of the plasma. With this simple method we can test different density gradient's effects on spectra in order to see if they would affect our results. This was done by running PrismSPECT with various densities, then creating single spectra from two spectra with different densities such that the average density and density gradient changed in measurable ways. This total transmission spectra was then convolved with our instrument functions and converted to optical depth. The He-like lines used in the Stark broadening investigation were then fitted with Voigt profiles so that their widths could be recorded. The average density, density gradient and widths were then used to create contour maps of line width with respect to average density and density gradient, as seen in figures 5.2 and 5.3.

From analysis of the $F-H e \delta$ line (figure 5.2) it appears that the sample could have been subject to a a large gradient, with variations between the front and the back of the plasma as high as $2^{21} e / c c$. Analysis of the $M g-H e \gamma$ also supports a gradient, but a much smaller one, closer to $7 \times 10^{20} \mathrm{e} / \mathrm{cc}$ difference between the front and back of the plasma. This amount of gradient would complicate our results, as it could lead to the discrepancies seen in our data. 


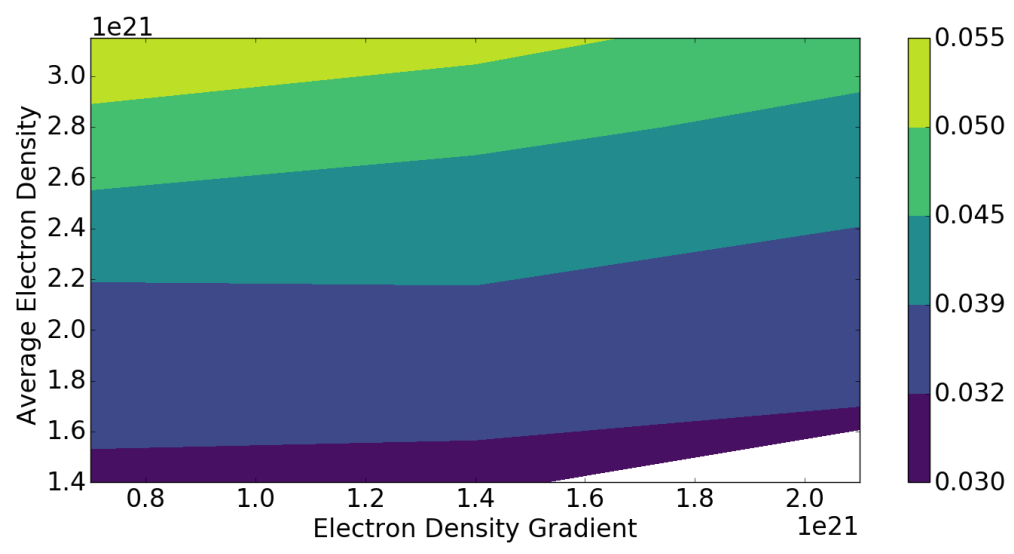

Figure 5.2: By simplifying a gradient to just two components, the effects of a gradient on a line's width becomes more apparent. Here, the width of the $F-H e \delta$ changes with both average density and density gradient. The range $0.032-0.039 \AA$ encompasses our experimental results (average density of $2.1 \times 10^{21}$ ). This line could have experienced a gradient.

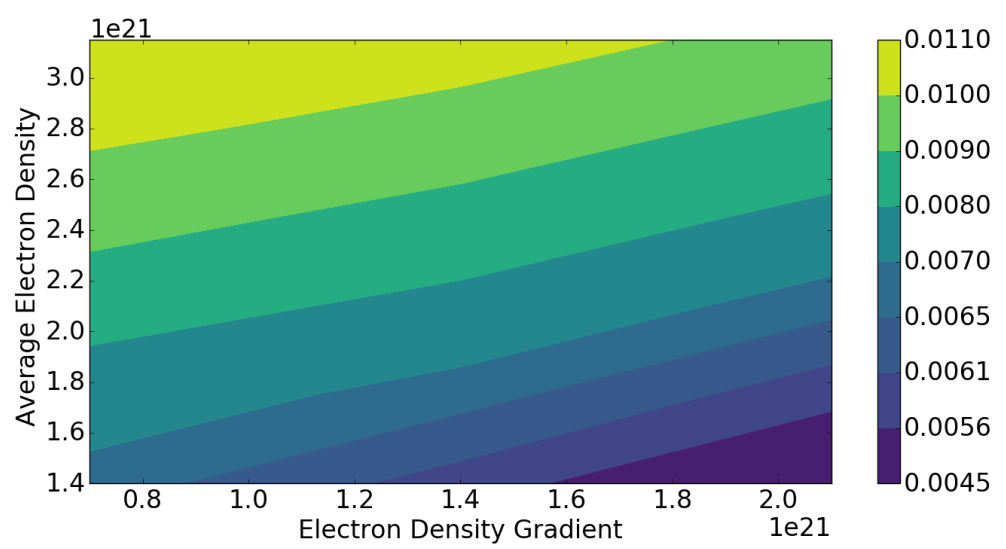

Figure 5.3: A similar pattern arises when looking at the $M g-H e \gamma$. The measurements corresponding to the $7 \mu m$ measurements is the $.0056-.0061 \AA$ interval. The investigation into gradients did not go to low enough densities to properly see if there is a gradient for this line, but an extrapolation to lower densities implies there is. Fortunately, this is a small range of gradients that would still supply the correct width and average density for our experimental results. 


\section{SUMMARY, CONCLUSIONS AND FUTURE WORK}

In order to properly determine how large a role density gradients play, a logical next step would be to perform new experiments. These experiments would attempt to maximize and minimize density gradients in the sample while also collecting transmission spectra and testing it against the spectra we have already collected.

\subsubsection{Temperature Gradients}

Similar to the line width investigation, the isoelectronic line ratio study could have a temperature gradient that may contaminate results. Temperature gradients are more complicated than density gradients, as the charge state distribution depends more highly on temperature than density. This means that a variation in temperature can impact the charge states present in the spectra, shifting to higher or lower depending on the magnitude of the temperature change.

Much like for the density gradients, we set up a simple two-temperature model to see the effects of a temperature gradient. For the temperature gradient, we just used one average temperature $(55 \mathrm{eV})$, and looked into three different gradients $0,10 \mathrm{eV}$ and $20 \mathrm{eV}$ from the front to the back of the sample. These spectra were convolved with PrismSPECT's inboard instrument function, a 900 $\frac{\lambda}{\Delta \lambda}$ Gaussian convolution in the interest of saving time. The resultant transmission spectra can be found in figures 5.4, 5.5 and 5.6.

Based on these spectra, the $\mathrm{F}$ lines used in the isoelectronic line ratio study do not change with a temperature gradient, as even a $20 \mathrm{eV}$ change did not affect the transmission from these lines enough to be seen in our data. This is most likely due to the fact that He-like ions change slowly as a function of temperature, due to the closed inner shell of electrons. These lines would not be very useful investigating gradients on their own. The Li-like $\mathrm{Mg}$ as well as the Be-like $\mathrm{Na}$ and $\mathrm{Mg}$ are susceptible to temperature gradients, as evidenced in the plots so line ratios using any of those lines, such as $M g-B e / N a-B e$ are the most useful to detect temperature gradients.

An experiment that would only deviate from our current version a small amount would be very easy to set up. All that would be needed would be new foils, where two of the last layers on one end were made to be $\mathrm{MgO}_{2}$, the middle 


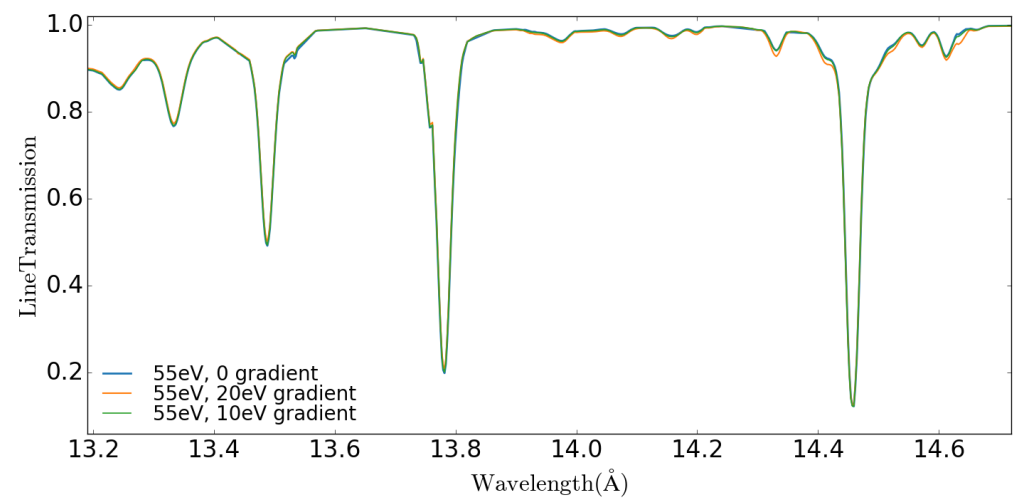

Figure 5.4: Even with a temperature gradient of $20 \mathrm{eV}$, the He-like $\mathrm{F}$ lines do not change much. This is due to the He-like lines changing slowly as a function of temperature.

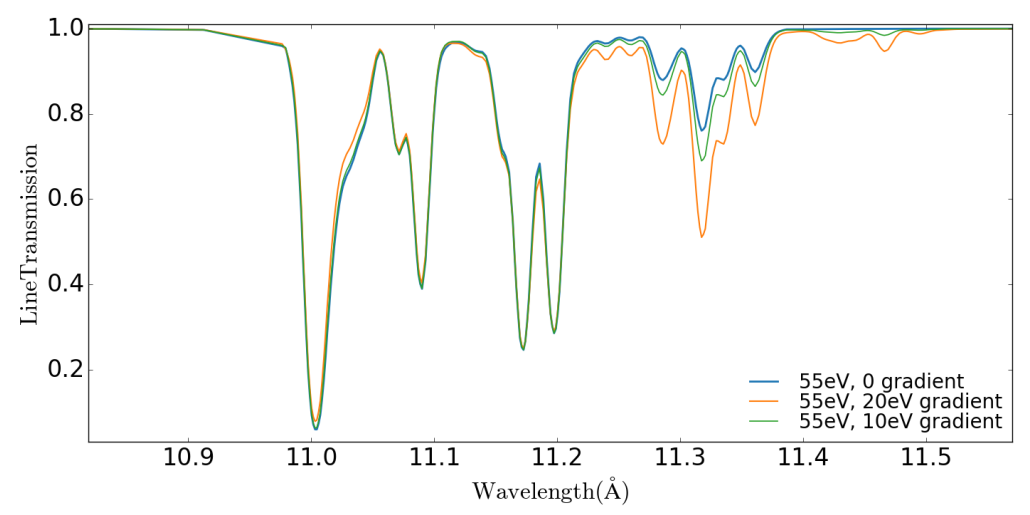

Figure 5.5: The Be-like $\mathrm{Na}$ at $11.3 \AA$ changes significantly with different temperature gradients, while the $H e \alpha$ at $11 \AA$ does not. 


\section{SUMMARY, CONCLUSIONS AND FUTURE WORK}

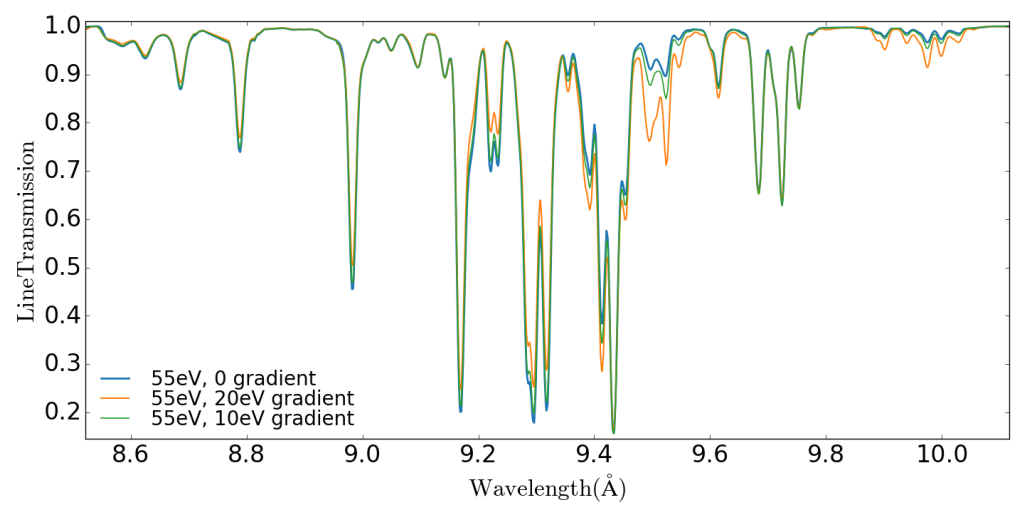

Figure 5.6: The He-like Na lines $(8.6-9 \AA)$ do not change much with a temperature gradient, but there is some change in the Li-like $(9.3 \AA)$ and the Be-like (9.4) Mg lines, meaning these lines could be useful in detecting a gradient.

would be only $N a F$ and the last two layers on the other end could be $A l$. Then several shots could be done with the $A l$ side facing the pinch. In this way, if isoelectronic line ratios involving $M g$ predicted lower temperatures than isoelectronic line ratios involving $A l$ then there would be a gradient, and the difference in predicted temperature between the two could be related to the amount of temperature gradient through the foil.

\subsubsection{Temporal Evolution}

The hardest possible future experiments are those dealing with temporal evolution of our sample. Due to the nature of the ZPDH, the backlighter is 3ns long, during which time the large amount of x-rays ( 220 TW of x-ray power) backlighting our sample may also be heating it further. This possible heating during the backlighter could lead to the plasma's temperature increasing and the electron density decreasing during the time we are trying to measure the spectra from our foils. Initial Radiation-Hydrodynamic simulations of our experiments indicate that this does happen for all tamper thicknesses (figure 5.7).

The best way to test this temporal evolution would be to take time-gated spectroscopic measurements. This could be done by using the Time-Resolved 


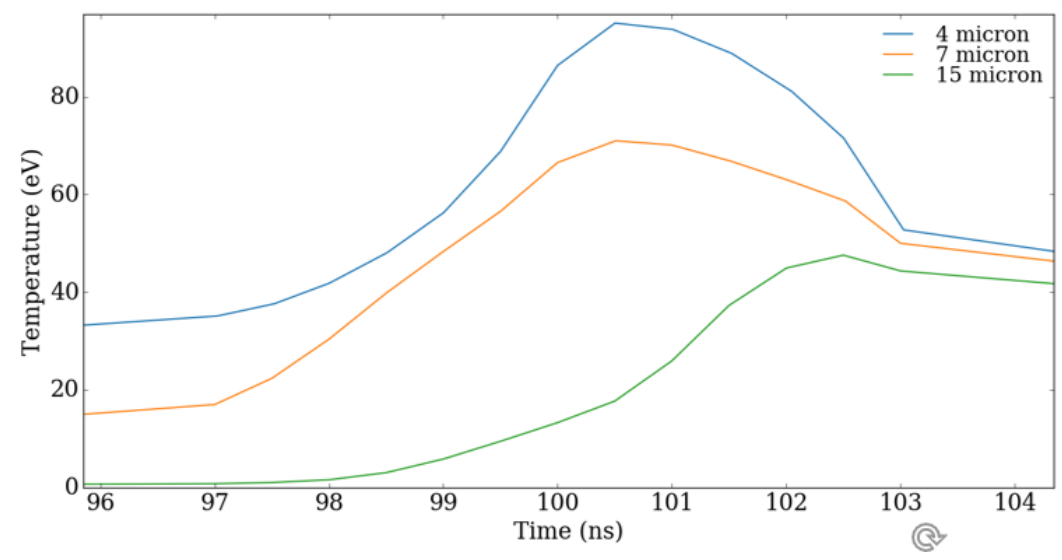

Figure 5.7: Running Helios (89) for our experimental setup, we find that Helios predicts a temporal evolution in temperature (and density) for our sample over the course of the pinch backlighter (100-103 ns).

Elliptical Spectrometer (T-REX) on Line of Sight (LOS) 330 at Z. However, this LOS is currently used by Roberto Mancini's group in ZAPP, so a trade for time on LOS 330 would need to be made, or separate, non-ZAPP shots would need to be planned out. This would require multiple shots, as the timing on T-REX has a certain amount of jitter that would need to be accounted for in timing, as well as multiple shots in order to satisfy statistical arguments for shot to shot error.

Another option would be conducting experiments with the Omega Laser at the Laboratory for Laser Energetics (LLE) in Rochester NY. The LLE has the capability to measure time-resolved spectra, as well as provide independent diagnostics for temperature and density. This could help resolve the question of gradients as well. This would require a considerable amount of time though, as it would require planning an entirely new experiment at a different facility, as well as getting time to use Omega, which can be challenging in itself. 
5. SUMMARY, CONCLUSIONS AND FUTURE WORK 


\section{Appendix A}

\section{Z Shot Dates}

Ths work put forth in this thesis comes from 10 different experimental runs on Sandia's Z machine. These shots were conducted over the course of three years, as shown in table A.1. During much of this time (May 2016-July 2018) the author lived in Albuquerque NM and worked on site at Sandia National Labs to aid in planning and processing these experiments. 


\begin{tabular}{l|l} 
Shot & Date \\
\hline & \\
z2950 & May 2016 \\
z2971 & June 2016 \\
z3053 & March 2017 \\
z3141 & September 2017 \\
z3194 & January 2018 \\
z3275 & July 2018 \\
z3276 & July 2018 \\
z3286 & August 2018 \\
z3364 & April 2019 \\
z3365 & April 2019
\end{tabular}

Table A.1: The list of shots on $\mathrm{Z}$ that yielded valuable spectra and the month and year they occurred. This helps illustrate how much time can pass in between Z shots. 


\section{Appendix B}

\section{List of Presentations}

Stewardship Science Academic Programs Symposium (SSAP)2019, Albuquerque NM, USA: (contributed poster) T. Lane, P. Kozlowski, M. Flaugh,T. Steinberger, M. Koepke, G. Loisel, G. Rochau, J. Bailey, A Methodology for Determining SelfConsistency of Stark Broadening Predictions in a Multi-Element HED Plasma. Received Outstanding Poster Award in the Graduate Student Poster Competition.

Naval Research Laboratory Radiation and Hydrodynamics Branch Seminar January 2019, Washington DC, USA: (invited colloquium) T. Lane, P. Kozlowski, M. Flaugh, T. Steinberger, M. Koepke, G. Loisel, G. Rochau, J. Bailey, A Methodology for Determining Self-Consistency of Stark Broadening Predictions in a Multi-Element HED Plasma

American Physical Society-Division of Plasma Physics Meeting (APS-DPP)2018, Portland OR, USA: (contributed talk) T. Lane, P. Kozlowski, M. Flaugh,T. Steinberger, M. Koepke, G. Loisel, G. Rochau, J. Bailey, A Methodology for Determining Self-Consistency of Stark Broadening Predictions in a Multi-Element HED Plasma

International Conference on Plasma Science (ICOPS) 2018, Denver CO, USA: (contributed poster) T. Lane, P. Kozlowski, G. Loisel, M. Flaugh, T. Steinberger, M. Koepke, J. Bailey, G. Rochau,Multi-Element Stark Broadening For Diagnosing Electron Density in HED Plasmas

Omega Laser Users Group (OLUG)2018, Rochester NY, USA: (contributed poster) T. Lane, P. Kozlowski, G. Loisel, M. Flaugh, T. Steinberger, M. Koepke, 


\section{B. LIST OF PRESENTATIONS}

J. Bailey, G. Rochau,Multi-Element Stark Broadening For Diagnosing Electron Density in HED Plasmas

High Energy Density Science Summer School (HEDSSS)2017, La Jolla CA, USA: (invited student talk) T. Lane, P. Kozlowski, M. Flaugh,T. Steinberger, M. Koepke, G. Loisel, G. Rochau, J. Bailey, Development of an Isoelectronic Line Ratio Temperature Diagnostic in Soft X-Ray Absorption Spectroscopy

International Conference on High Energy Density Physics (ICHED)2017, Shirahama Wagayama, Japan: (contributed talk) T. Lane, P. Kozlowski, M. Flaugh,T. Steinberger, M. Koepke, G. Loisel, G. Rochau, J. Bailey, Development of an Isoelectronic Line Ratio Temperature Diagnostic in Soft X-Ray Absorption Spectroscopy

Omega Laser Users Group (OLUG)2017, Rochester NY, USA: (contributed poster) T. Lane, P. Kozlowski, M. Flaugh,T. Steinberger, M. Koepke, G. Loisel, G. Rochau, J. Bailey, Development of an Isoelectronic Line Ratio Temperature Diagnostic in Soft X-Ray Absorption Spectroscopy

International Conference on Plasma Science (ICOPS) 2016, Banff Alberta, Canada: (contributed talk) T. Lane, M. Flaugh,T. Steinberger, M. Koepke, G. Loisel, G. Rochau, J. Bailey, Development of an Isoelectronic Line Ratio Technique For Improved Diagnosing of Temperature and Temperature Gradients in Photoionized Plasma

Omega Laser Users Group (OLUG)2016, Rochester NY, USA: (contributed poster) T. Lane. M. Flaugh,T. Steinberger, M. Koepke, G. Loisel, G. Rochau, $\mathrm{J}$. Bailey, Improving the fidelity of interpreting time-averaged spectra on $\mathrm{Z}$ for development of a multi-element Stark-broadening diagnostic.. Received Honorary Mention in the Graduate Student Poster Competition

Interrelation between Plasmas in Experiment and Space (IPELS) 2015, Pitlochry Scotland, UK: (invited talk) T. Lane Scientific Collaboration and Capability at the astronomical-observation and laboratory plasma Interface: The Z Astrophysical Plasma Properties (ZAPP) Collaboration

Radiation and High Energy Density Plasmas (RHEDP) 2015, Lake Tahoe NV, USA: (contributed poster) T. Lane, M. Flaugh, M. Koepke, G. Loisel, G. Rochau, J. Bailey, Assessing LTE reference conditions in photoionized-plasma experiments 
using a NaF-foil target to resolve charge-state-distribution discrepancies between models

Omega Laser Users Group (OLUG)2015, Rochester NY, USA: (contributed poster) T. Lane. M. Flaugh, M. Koepke, G. Loisel, G. Rochau, J. Bailey, Establishing LTE reference conditions for photo-ionized plasma experiments using thick tamped sodium-fluoride foil target. Received Honorary Mention in the Graduate Student Poster Competition

Resonant Auger Destruction in Photoionized Silicon Workshop 2015, Albuquerque NM, USA: (invited talk) T. Lane, M. Flaugh, M. Koepke, G. Loisel, G. Rochau, J. Bailey, X-ray drive characterization -Experiment: What can we learn from the LTE opacity platform

SLAC-LCLS High Power Laser Science Workshop 2014, Palo Alto CA, USA: (contributed poster) T. Lane, M. Flaugh, M. Koepke, G. Loisel, G. Rochau, J. Bailey, D. Liedahl, D. Winget, R. Mancini, WVUs contributions to the Z Astrophysical Plasma Properties (ZAPP) project on Sandias Z facility 
B. LIST OF PRESENTATIONS 


\section{Appendix C}

\section{Example of Code}

Below is an example of a code written for the work done in this thesis. This specific code, titled convert_optdepth_linefit_prism_all.pro was used to fit a Voigt profile to spectral features generated by PrismSPECT. This code was written in IDL and calls the function vgl_line_fit_trans_prism.pro, which is a function originally written by G. Loisel and modified by T.S. Lane for the purposes of this thesis. This code fits a Lorentzian, a Gaussian and a Voigt profile to a spectral feature for all of the PrismSPECT spectra, and outputs the Voigt fits' area, width and standard deviation of area and width.

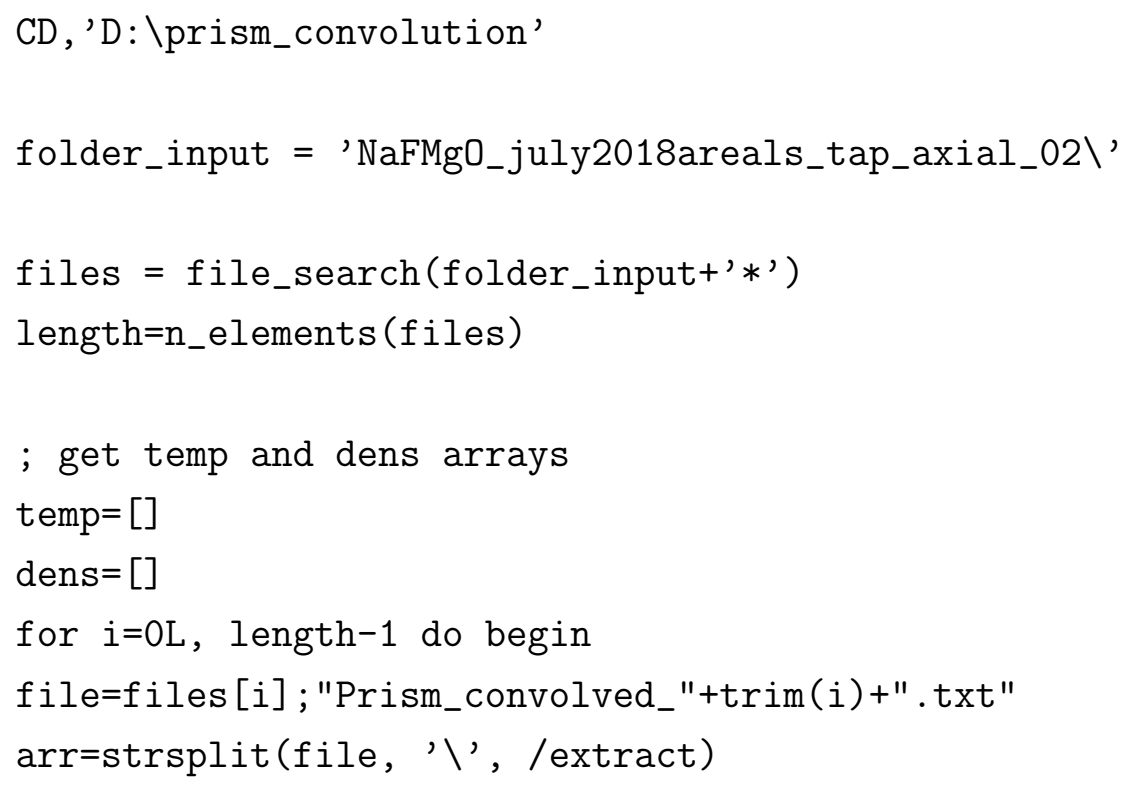




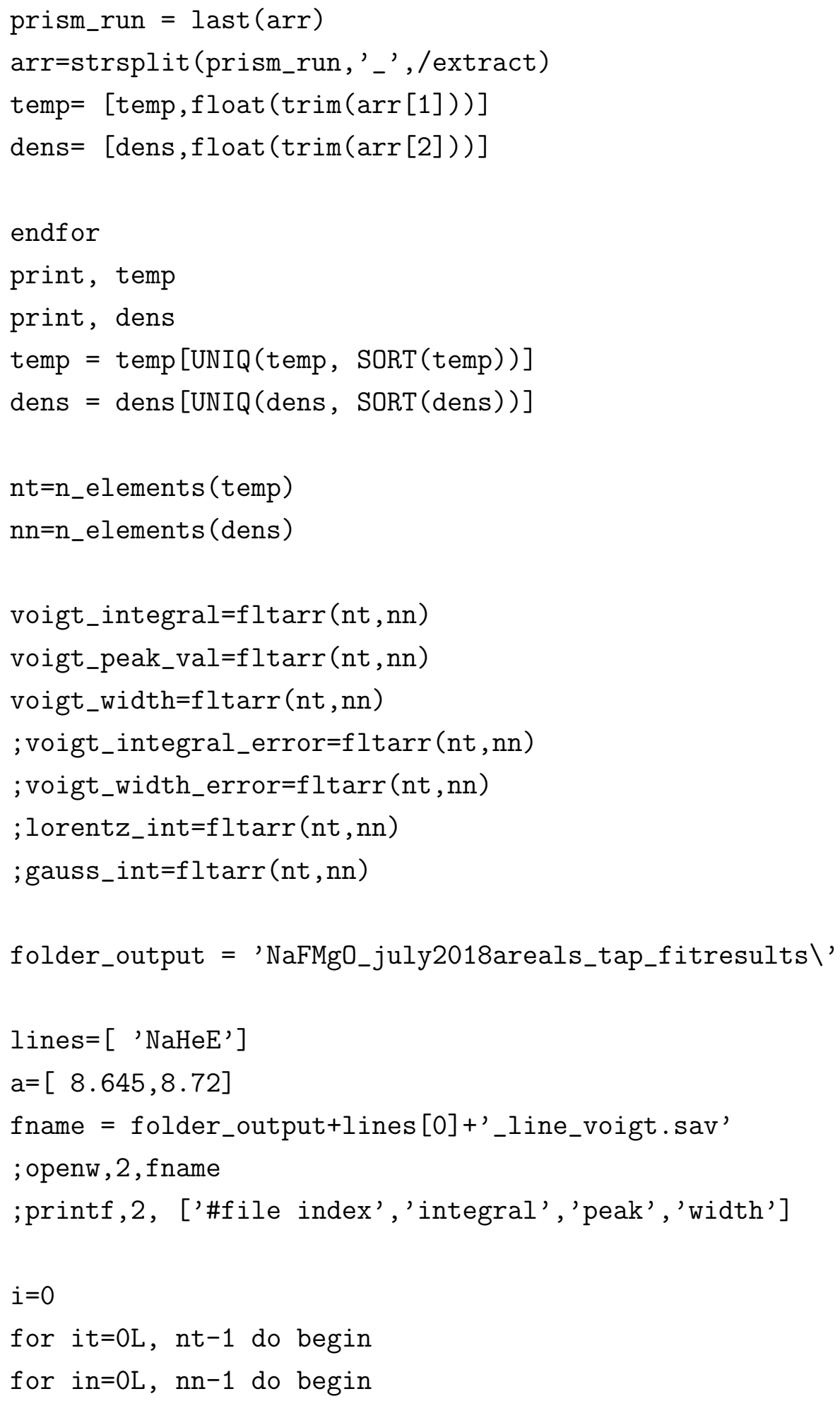




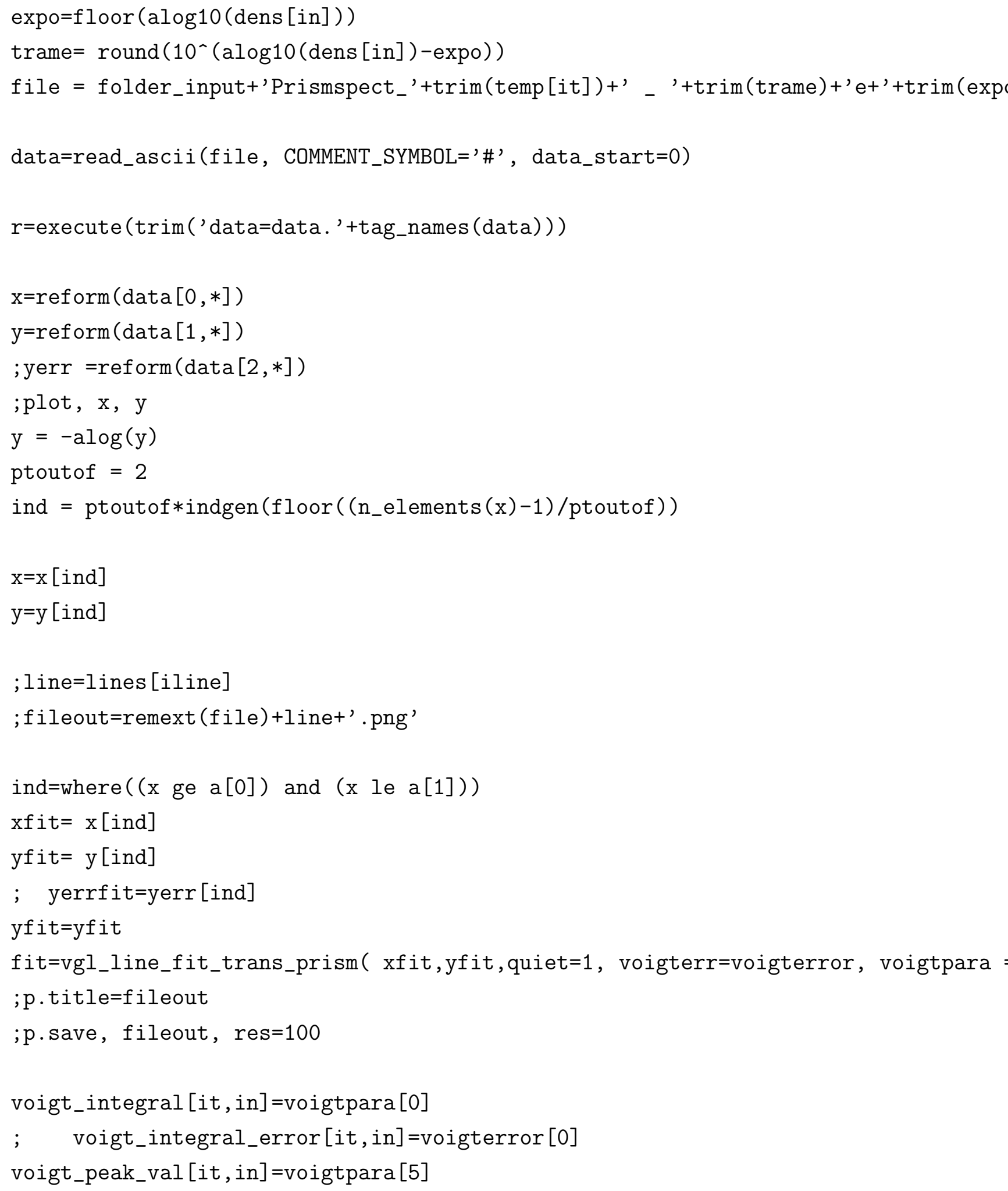




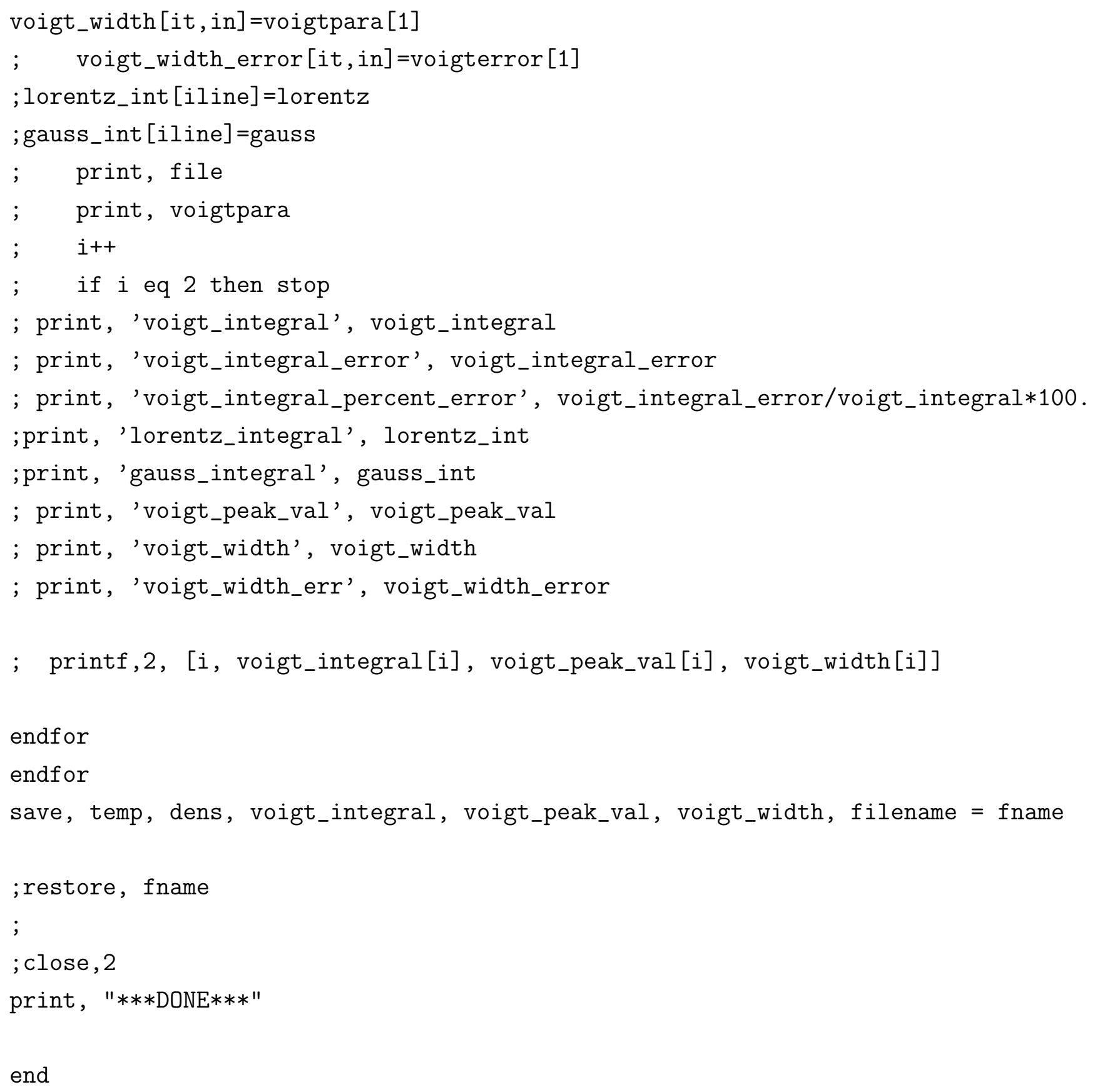




\section{References}

[1] M. Barrios, D. Liedahl, M. Scheider, O. Jones, G. Brown, et al. Electron temperature measurements inside the ablating plasma of gas-filled hohlraums at the National Ignition Facility. Physics of Plasmas, 23(5), 056307 (2016). 1, 54

[2] H. R. Griem, A. C. Kolb, K. Shen. Stark Broadening of Hydrogen Lines in a Plasma. Physical Review, 116(4) (1959). 1

[3] J. Bailey, T. Nagayama, G. Loisel, G. Rochau, C. Blancard, et al. A higherthan-predicted measurement of iron opacity at solar interior temperatures. Nature, $\mathbf{5 1 7}(14048), 56$ (2015). 1, 53, 55

[4] J. Bailey, G. Rochau, R. Mancini, C. Iglesias, J. MacFarlane, et al. Diagnosis of x-ray heated $\mathrm{Mg} / \mathrm{FE}$ opacity research plasmas. Review of Scientific Instruments, 79(11), 113104 (2008). 1, 34

[5] M. A. Gigosos. Stark broadening models for plasma diagnostics. Journal of Physics D: Applied Physics, 47(34), 343001 (2014). 1

[6] M. Qian, C. Ren, D. Wang, J. Zhang, G. Wei. Stark broadening measurement of the electron density in an atmospheric pressure argon plasma jet with double-power electrodes. Journal of Applied Physics, 107(6), 063303 (2010). 1

[7] T. Nagayama, J. Bailey, G. Loisel, G. Rochau, J. MacFarlane, et al. Calibrated Simulations of $\mathrm{Z}$ opacity experiments that reproduce the experimentally measured plasma condictions. Physical Review E, 93(2), 023202 (2016). 2 


\section{REFERENCES}

[8] R. P. Drake. High Energy Density Physics: Fundementals, Inertial Fusion and Experimental Astrophysics, vol. 1, (Springer2006). 3

[9] T. Fujimoto. Plasma spectroscopy, vol. 123, (Oxford University Press2004). $4,5,8,11,19$

[10] J. Bauche, C. Bauche-Arnoult, O. Peyrusse. Atomic Properties in Hot Plasmas: From levels to Superconfigurations, vol. 1, (Springer2015). 4, 5

[11] H. Griem. Spectral Line Broadening by Plasmas. 1, (Academic Press1974). 4,6

[12] H.-J. Kunze. Introduction to plasma spectroscopy, vol. 56, (Springer2009). $5,6,9,11,12,13,18,54,85$

[13] D. Salzmann. Atomic physics in hot plasmas. 97, (Oxford University Press1998). 6, 9, 10, 11, 16, 18, 21, 22

[14] M. Baranger. General Impact Theory of Pressure Broadening. Physical Review, 112(3), 855 (1958). 8

[15] C. Kittel, H. Kroemer. Thermal Physics. 2, (MacMillan1980). 9

[16] H. R. Griem. Validity of Local Thermodynamic Equilibrium in Plasma Spectroscopy. Physical Review, 131(3), 1170 (1963). 10

[17] T. Fujimoto, R. McWhirter. Validity criteria for local thermodynamic equilibrium in plasma spectroscopy. Physical Review A, 42(11), 6588 (1990). 10

[18] T. Kallman. XSTAR: A Spectral Analysis Tool. 2.5, (NASA Goddard2018). 10

[19] A. Kramida, J. Fuhr. Atomic Transition Probability Bibliographic Database. 9, (National Institute of Standards and Technology2019). 12

[20] G. Martin, W. Wiese. Atomic oscillator-strength distributions in spectral series of the lithium isoelectronic sequence. Physical Review A, 13(2), 699 (1976). 14 
[21] H. Kramers. XCIII. On the theory of X-ray absorption and of the continuous X-ray spectrum. Philosophical Magazine and Journal of Science, 46(275), 836 (1923). 14

[22] D. J. Botto, J. McEnnan, R. Pratt. Analytic description of photoeffect from atomic ions. Physical Review A, 18(2), 580 (1978). 14

[23] D. Osterbrock. Astrophysics of Gaseous Nebulae and Active Galactic Nuclei. 1, (University Science Books1989). 14

[24] H. Van Regemorter. Rate of Collisional Excitation in Stellar Atmospheres. Astrophysical Journal, 136, 906 (1962). 17

[25] D. H. Sampson, H. L. Zhang. Use of Van Regemorter formula for collision strengths or cross sections. Physical Review A, 45(3), 1556 (1992). 17

[26] L. Golden, D. Sampson. Ionization of highly charged ions by electron impact. Journal of Physics B, 10(11), 2229 (1977). 18

[27] L. Golden, D. Sampson. Electron impact results by he $\mathrm{Z}=$ infinity method. Journal of Physics B, 11(3), 541 (1978). 18

[28] L. Golden, D. Sampson. Ionisation from the 4s, 4p, 4d and $4 \mathrm{f}$ sublevels of highly charged ions. Journal of Physics B, 13(13), 2645 (1980). 18

[29] S. Younger. Electron-impact ionization cross sections for highly ionized hydrogen- and lithium-like atoms. Physical Review A, 22(1), 111 (1980). 20

[30] S. Younger. Electron-impact-ionization cross sections for highly ionized helium-like ions. Physical Review A, 22(4), 1425 (1980). 20

[31] S. Younger. Electron-impact-ionization cross sections and rates for highly ionized beryllium-like ions. Physical Review A, 24(3), 1278 (1981). 20

[32] A. Gabriel. Dielectric Satellite Spectra for Highly-Charged Helium-Like Ion Lines. Monthly Notices of the Royal Astronomical Society, 160(1), 99 (1972). 23 


\section{REFERENCES}

[33] I. Beigman, B. Chichkov. Dielectric recombination through the forbidden levels. Journal of Physics B, 13(3), 565 (1980). 23

[34] V. Jacobs, J. Cooper, S. Haan. Unified description of radiative and dielectronic recombination, including the coupling between autoionization and radiation continua. Physical Review A, 36(3), 1093 (1987). 23

[35] U. Safronova, A. S. Safronova, P. Beiersdorfer. Excitation energies, radiative and autoionization rates, dielectronic satellite lines, and dielectronic recombination rates for excited states of Yb-like W. Journal of Physics B, 45(8), 085001 (2011). 23

[36] R. Spielman, S. Breeze, C. Deeney. PBFA Z: A 20-MA z-pinch driver for plasma radiation sources. Conference (1996). 25

[37] W. H. Bennett. Magnetically Self-Focussing Streams. Physical Review, 45(12). 25

[38] E. Marx. Experiments on the Testing of Insulators using High Voltage Pulses. Elektrotechnische Zeitschrift, 45, 652 (1924). 25

[39] T. W. OSanford. Overview of the Dynamic-Hohlraum X-ray Source at Sandia National Laboratories. 1, (Sandia National Laboratories2007). 25

[40] G. Rochau, J. Bailey, R. Falcon, G. Loisel, T. Nagayama, et al. ZAPP: The Z Astrophysical Plasma Properties collaboration. Physics of Plasmas, 21(5), 056308 (2014). 26, 27, 29

[41] G. Loisel. personal communication. 26

[42] G. Rochau, J. Bailey, J. MacFarlane. Measurement and analysis of x-ray absorption in $\mathrm{Al}$ and $\mathrm{MgF}$ plasmas heated by Z-pinch radiation. Physical Review E, 72(6), 066405 (2005). 25

[43] G. Loisel, J. Bailey, D. Liedahl, C. Fontes, T. Kallman, et al. Benchmark Experiment for Photoionized Plasma Emission from Accretion-Powered Xray Sources. Physical Review Letters, 119(7), 075001 (2017). 25 
[44] G. Loisel, J. Bailey, T. Nagayama, G. Rochau. Manuscript concerning Z Pinch reradiation. unpublished. 28

[45] P. Kozlowski, T. Lane, M. Koepke, G. Loisel, J. Bailey, et al. Manuscript concerning Z Pinch reradiation experiments. unpublished. 28

[46] G. Loisel. personal communication. 28

[47] T. Nash, M. Derzon, G. Chandler, D. Fehl, R. Leeper, et al. Diagnostics on Z (invited). Review of Scientific Instruments, 72(1), 1167 (2001). 29

[48] G. Loisel, J. Bailey, G. Rochau, G. Dunham, L. Nielsen-Weber, et al. A methodology for calibrating wavelength dependent spectral resolution for crystal spectrometers. Review of Scientific Instruments, 83(10), 10E133 (2012). 32

[49] M. Swartz, S. Kastner, E. Rothe, W. Neupert. Soft x ray spectra of Cr, Mn, $\mathrm{Fe}, \mathrm{Co}, \mathrm{Ni}, \mathrm{Cu}$; classifications in Ne I, F I, O I, N I and C I isoelectronic sequences. Journal of Physics B, 4(12), 1747 (1971). 39

[50] T. Perry, P. Springer, D. Fields, D. Bach, F. Serduke, et al. Absorption experiments on x-ray heated mid-Z constrained samples. Physical Review E, $\mathbf{5 4}(5), 5616$ (1996). 41, 53

[51] E. Henke, BL Gullikson, J. Davis. X-ray interactions: photoabsorption, scattering, transmission and reflection at $\mathrm{E}=50-30000 \mathrm{eV}, \mathrm{Z}=1-92$. Atomic Data and Nuclear Data Tables, 54(2), 181 (1993). 46

[52] R. S. Marjoribanks, F. Budnik, G. Kulcsar, L. Zhao. Isoelectronic line intensity ratios for plasma electron temperature measurement (invited). Review of Scientific Instruments, 66(1), 683 (1995). 53

[53] T. Nagayama, J. Bailey, G. Loisel, S. Hansen, G. Rochau, et al. Control and diagnosis of temperature, density and uniformity in x-ray heated iron/magnesium samples for opacity measurements. Physics of Plasmas, 21(5), 056502 (2014). 53, 55 


\section{REFERENCES}

[54] X. Xiangdong, W. Cheng, H. Shensheng, X. Zhizhan. Inter-stage line ratio of He- and Li-like Ti emissions for the electron temperature measurement. Plamsa Science and Technology, 7(2), 2764 (2005). 53, 55

[55] C. Keane, B. Hammel, D. Kania, J. Kilkenny, R. Lee, et al. X-ray spectroscopy of high-energy density inertial confinement fusion plasmas. Physics of Fluids B, 5(9), 3328 (1993). 53, 55

[56] S. McIntosh, J. Brown, P. Judge. The relation between line ratio and emission measure analyses. Astronomy and Astrophysics, 333, 333 (1998). 53

[57] C. Back, S. Glenzer, R. Lee, B. MacGowan, J. Moreno, et al. Spectroscopic Temperature Measurements of Non-Equilibrium Plasmas. AIP Conference Proceedings, 381(1), 123 (1996). 53

[58] T. Shepard, C. Back, D. Kalantar, R. Kauffman, C. Keane, et al. T measurements in open- and closed-geometry long-scale-length laser plasmas via isoelectronic x-ray spectral line ratios. Review of Scientific Instruments, 66(1), 749 (1995). 53

[59] R. Marjoribanks, M. Richardson, P. Jaanimagi, R. Epstein. Electrontemperature in laser-produced plasmas by the ratio of isoelectronic line intensities. Physical Review A, 46(4), R1747 (1992). 53, 60

[60] C. Back, D. Kalanta, R. Kauffman, R. Lee, B. MacGowan, et al. Measurements of Electron Temperature by Spectroscopy in Hohlraum Targets. Physical Review Letters, 77(21), 4350 (1996). 54, 55, 60

[61] F. Budnik, G. Kulcsar, L. Zhao, R. s. Marjoribanks, H. Chen, et al. Laser/plasma studies using the terawatt FCM-CPA laser at Toronto. Proceedings of SPIE, 2041 (1994). 54

[62] T. Shepard, C. Back, D. Kalantar, R. Kauffman, C. Keane, et al. Isoelectronic X-ray spectroscopy to determine electron temperatures in long-scalelength inertial-confinement-fusion plasmas. Physical Review E, 53(5), 5291 (1996). 54 
[63] J. Olivero, R. Longbothum. Empirical fits to the Voigt line width: A brief review. Journal of quantitative Spectroscopy and Radiative Transfer, 17(2), 233 (1977). 59

[64] J. MacFarlane, I. Golovkin, P. Wang, P. Woodruff, N. Pereya. SPECT3D A Multi-Dimensional Collisional-Radiative Code for Generating Diagnostic Signatures Based on Hydrodynamics and PIC Simulation Output. High Energy Density Physics, 3(1-2), 181 (2007). 60

[65] J. MacFarlane, I. Golovkin, P. Woodruff, D. Welch, B. Oliver, et al. Simulation of the ionization dynamics of aluminum irradiated by intense short-pulse lasers. Inertial Fusion Sciences and Applications, 457-460 (2004). 60

[66] P. CS. Prism Atomic Data Overview. online (2017). 62

[67] H. R. Griem, M. Blaha, P. C. Kepple. Stark-profile calculations for Lymanseries lines of one-electron ions in dense plasmas. Physical Review A, 19(6), 2421 (1979). 78

[68] I. E. Golovkin. personal communication. 78

[69] R. Mancini, D. Kilcrease, L. Woltz, C. Hooper Jr. Calculational aspects of the Stark line broadening of multielectron ions in plasmas. Computational Physics Communications, 63(1), 314 (1991). 78

[70] H. R. Griem. Spectral Line Broadening by Plasmas, vol. 1, (Academic Press1974). 78

[71] C. Hooper Jr. Electric Microfield Distributions in Plasmas. Physical Review, 149(1), 77 (1966). 78

[72] C. Hooper Jr. Electric Microfield Distributions in Plasmas. Physical Review, 165(1), 215 (1968). 78, 79

[73] L. Woltz, C. Hooper Jr. Calculation of spectral line profiles of multielectron emitters in plasmas. Physical Review A, 38(9), 4766 (1988). 79 


\section{REFERENCES}

[74] R. J. Tighe, C. Hooper Jr. Stark broadening in hot, dense laser-produced plasmas. Physical Review A, 14(4), 1514 (1976). 80, 99

[75] C. Fontes, H. Zhang, J. Abdallah Jr, R. Clark, D. Kilcrease, et al. The Los Alamos suite of relativistic atomic physics codes. Journal of Physics B, 48(14), 144014 (2015). 80

[76] J. Abdullah Jr, R. Clark, R. Cowan. Theoretical atomic physics code development I: CATS: Cowan Atomic Structure Code. Technical Report (1988). 80

[77] R. Clark, J. Abdullah Jr, G. Csanak, J. Mann, R. Cowan. Theoretical atomic physics code development II: ACE: Another Collisional Excitation Code. Technical Report (1988). 80

[78] S. J. Adelman, W. Wiese. Astrophysical applications of powerful new databases. Astronomical Societry of the Pacific Conference Series, Proceedings of Joint Discussion, 78(16) (1995). 80

[79] J. Colgan, D. Kilcrease, N. Magee, M. Sherrill, J. Abdallah Jr, et al. A new generation of Los Alamos opacity tables. The Astrophysical Journal, $\mathbf{8 1 7}(116)$ (2016). 80

[80] R. Lee. Plasma lines shapes for selected transitions in hydrogen-, heliumand lithium-like ions. Journal of Quantitative Spectroscopy and Radiative Transfer, 40(5), 561 (1988). 80, 81, 99

[81] C. A. Iglesias, J. L. Lebowitz, D. MacGowan. Electric microfield distributions in strongly coupled plasmas. Physical Review A, 28(3), 1667 (1983). 80

[82] J. W. Dufty, D. B. Boercker, C. A. Iglesias. Electric microfield distributions in strongly coupled plasmas. Physical Review A, 31(3), 1681 (1985). 80, 81

[83] W. Press, S. Teukolsky, W. Vetterling, B. Flannery. Numerical Recipes: The Art of Scientific Computing, vol. 3, (Academic Press2007). 81

[84] W. Karza, R. Latter. Electron Radiative Transitions in a Coulomb Field. Astrophysical Journal supplement, 6, 167 (1961). 99 
[85] H.-K. Chung, M. Chen, W. Morgan, Y. Ralchenko, R. Lee. FLYCHK: Generalized population kinetics and spectral model for rapid spectroscopic analysis for all elements. High Energy Density Physics, 1(1), 3 (2005). 105

[86] Y. Ralchenko, Y. Maron. Accelerated recombination due to resonant deexcitation of metastable states. Journal of Quantitative Spectroscopy and Radiative Transer, 71(2-6), 609 (2001). 105

[87] S. Hansen, J. Bauche, C. Bauche-Arnoult, M. Gu. Hybrid atomic models for spectroscopic plasma diagnostics. High Energy Density Physics, 3(1-2), 109 (2007). 105

[88] T. A. Gomez. Improving Calculations of the Interaction Between Atoms and Plasma Particles and Its Effect on Spectral Line Shapes. Ph.D. thesis, University of Texas at Austin (2017). 105

[89] J. Macfarlane, I. Golovkin, P. Woodruff. HELIOS-CR-a 1-D radiationmagnetohydrodynamics code with inline atomic kinetics modeling. Journal of Quantitative Spectroscopy and Radiative Transer, 99(1-3), 381 (2006). 111 